\title{
A RANDOMISED COMPARISON OF REGULAR ORAL OXYCODONE AND INTRATHECAL MORPHINE FOR POST-CAESAREAN ANALGESIA
}

\author{
Nolan John McDonnell BHB MBChB FANZCA \\ This thesis is presented in partial fulfillment of the requirements for the degree of \\ Master of Clinical Research of the University of Western Australia \\ School of Medicine and Pharmacology \\ Faculty of Medicine, Dentistry and Health Sciences
}

The University of Western Australia

October 2009 


\begin{abstract}

\section{Introduction}

A variety of techniques are available for post-caesarean analgesia. As the number of caesarean deliveries increase in developed countries, so too does the research into post-caesarean pain. Opioids form the foundation of most multimodal analgesia regimens post-caesarean delivery but they have not been adequately studied by the oral route of administration. This approach has the potential to show an improved side effect profile compared to other routes of administration, greater maternal acceptability as well as potentially avoiding some of the complications associated with neuraxial based techniques.
\end{abstract}

\begin{abstract}
Aims
This thesis has evaluated primary post-caesarean analgesia based on an oral opioid regimen with that provided by intrathecal morphine among women undergoing caesarean delivery under spinal anaesthesia. The secondary objectives included an evaluation of the side effects and acceptability of a multimodal analgesic regimen based on regular oral oxycodone.
\end{abstract}

\title{
Methods
}

After obtaining Institutional Ethics Committee approval and written informed consent, a double blind, double dummy, placebo controlled clinical trial was performed in which 120 women were randomised to receive either sustained-release oral oxycodone $20 \mathrm{mg}$ in the recovery room followed by immediate-release oxycodone $10 \mathrm{mg}$ 6-hourly for the first $24 \mathrm{~h}$ (Group O) or intrathecal morphine 100 mcg at the time of spinal anaesthesia (Group I). All women received regular postoperative diclofenac, paracetamol and standardised supplemental analgesia. The null hypothesis was that there would be no difference between the groups with respect to early post-operative analgesia. The primary endpoint for the study was the area under the curve for dynamic pain over the first 24 hours. The analysis was performed using SPSS 15.0 for Windows (SPSS, Chicago, IL).

\section{Results}

One hundred and eleven women completed the study. The characteristics of the two patient groups were similar. The area under the curve for pain scores to $24 \mathrm{~h}$ 
did not differ significantly between groups for pain at rest $(P=0.47)$ or on movement $(P=0.53)$. Numerical pain scores were low and similar, except at rest at $12 \mathrm{~h}$ (group I 1 [0-2] vs. group O 2 [1-3]; $P=0.03$ ). The time to first analgesic request was similar but additional post-operative analgesics were required more often in group $\mathrm{O}(82 \%$ vs. $63 \%, P=0.03)$. Group $\mathrm{O}$ more frequently reported that their worst pain scores were higher (score $4-10$ in $87 \%$ vs. $64 \%, P=0.007$ ). Pruritus was more common and more severe in group I ( $87 \%$ vs. $56 \%, P=0.001)$. There were no significant differences between groups in the incidence of nausea, epigastric pain, urinary retention or sedation scores. At $24 \mathrm{~h}$ maternal satisfaction with the analgesic regimen was lower in group $\mathrm{O}$ (score $8[7-10]$ vs. $10[8-10])(P=0.010)$, but this difference was no longer apparent at $48 \mathrm{~h}$ (score 9[8-9] vs. 9[8-10]) in Groups O and I respectively $(\mathrm{P}=0.89)$.

\section{Conclusions}

The results of this study indicate that post-operative pain relief after caesarean section from a regimen based on oral oxycodone is not significantly different to that from a regimen based on intrathecal morphine. Oral oxycodone was associated with a lower incidence of pruritus but also with a lower maternal satisfaction score for the first 24 hours post-operatively. The intrathecal morphine regimen appeared to have some efficacy advantages which we postulate were due to comparatively less episodic pain in the women receiving intrathecal morphine. A multimodal analgesic regimen based on oral oxycodone is an acceptable approach to post-caesarean analgesia. There is considerable potential to refine the oral oxycodone regimen and future studies should be conducted in this regard. The economic implications of such an approach also deserve consideration. 


\section{Table of Contents}

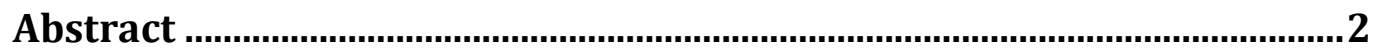

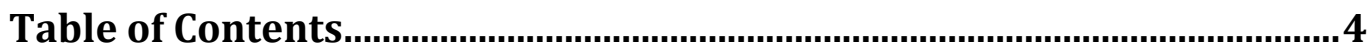

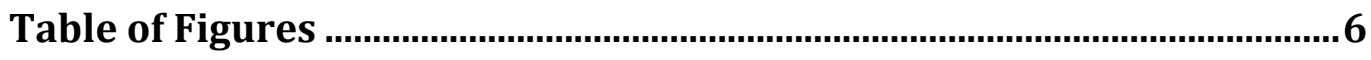

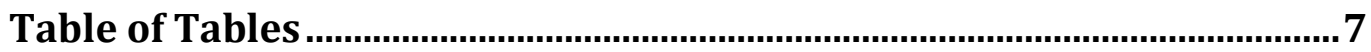

Acknowledgements ......................................................................................

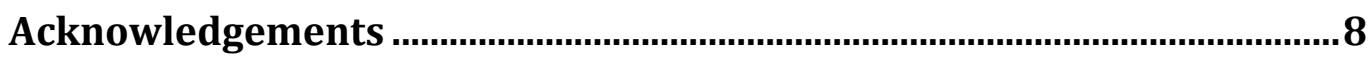

Publications, Abstracts and Presentations Arising from this Thesis.... 10

Abbreviations …................................................................................. 11

Statement of candidate contribution ..................................................... 12

1. Chapter One: Literature Review...................................................... 15

1.1 Introduction............................................................................................................15

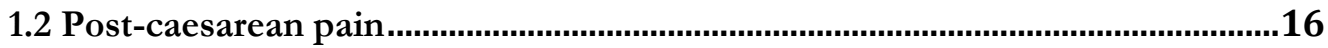

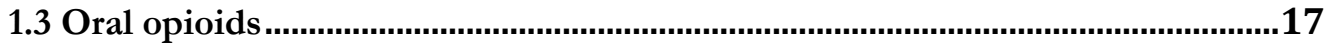

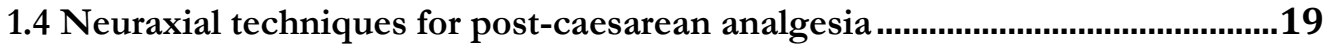

1.4.1 Spinal/Intrathecal opioids ……………………………………………………. 19

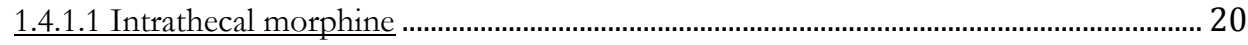

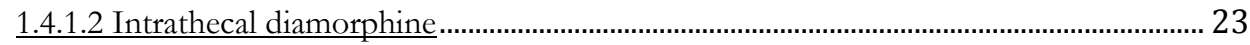

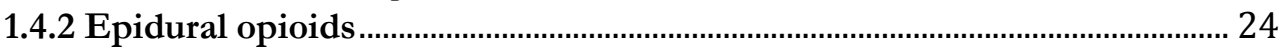

1.4.2.1 Epidural morphine .......................................................................................................... 24

1.4.2.2 Epidural diamorphine.......................................................................................................... 29

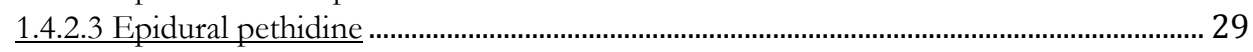

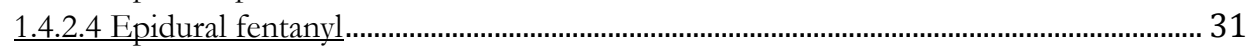

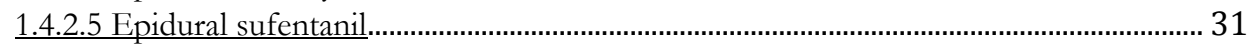

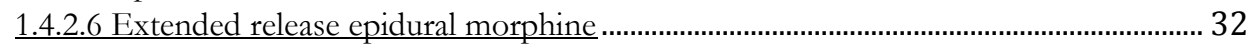

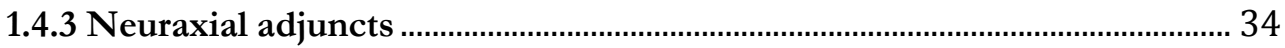

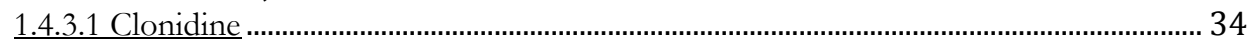

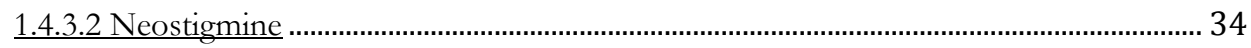

1.5 Intravenous, intramuscular and subcutaneous opioids ………………………...36

1.6 Non steroidal Anti-inflammatory Drugs................................................................37

1.7 Paracetamol......................................................................................................38

1.8 Wound infiltration and nerve blocks...................................................................38

1.9 Chronic post-caesarean pain ................................................................................43

1.10 Conclusions ..................................................................................................44

2. Chapter Two: Primary Oral Analgesia Post- Caesarean Delivery.. 45

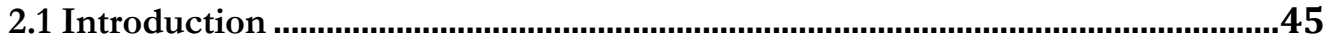

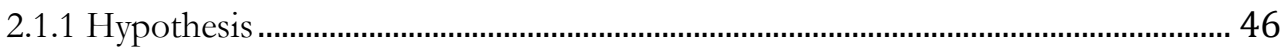

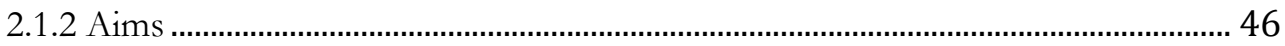

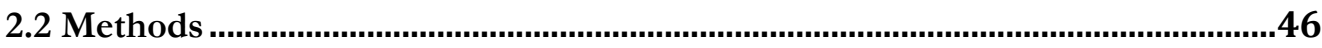

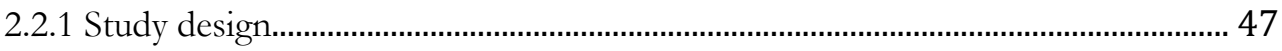

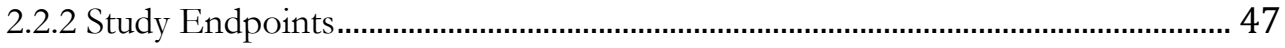

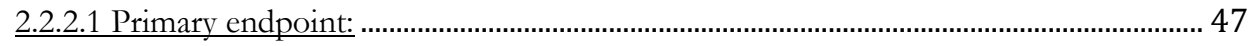

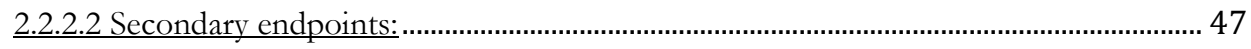

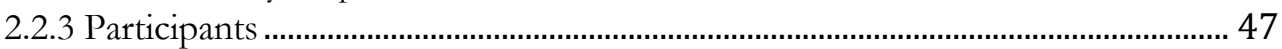

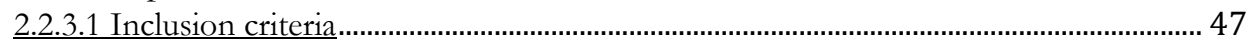

2.2.3.2 Exclusion criteria ............................................................................................................ 48

2.2.4 Group assignment and study drugs...................................................................... 48

2.2.5 Pre-operative preparation ............................................................................................ 49 
2.2.6 Management of anaesthesia and analgesia............................................................ 49

2.2.7 Post-operative management........................................................................ 50

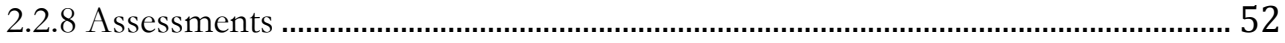

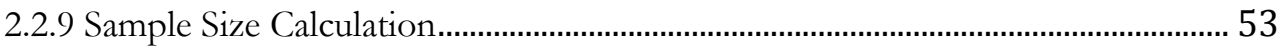

2.2.10 Statistical Methods........................................................................................ 53

2.2.11 Ethical issues............................................................................................... 54

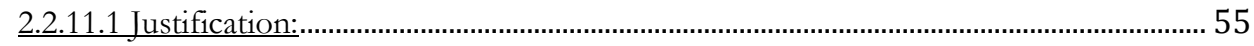

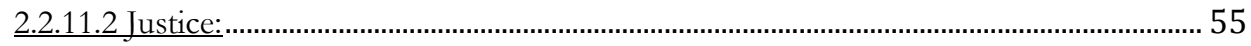

2.2.11.3 Beneficence: ……………………………………………………………………. 55

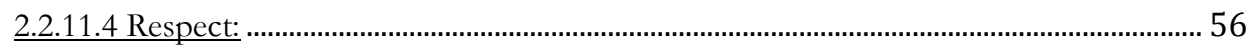

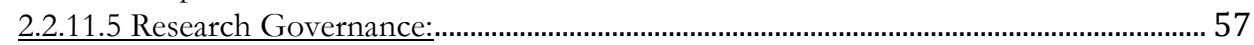

2.2.12 Funding sources and budget..................................................................... 57

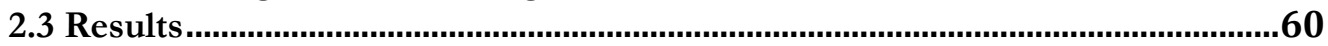

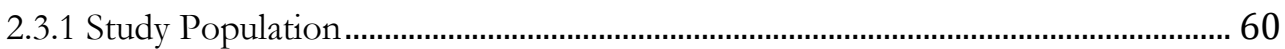

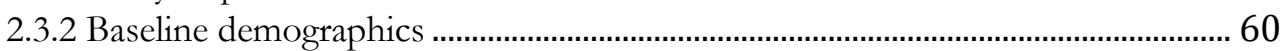

2.3.3 Primary and Secondary Endpoints .......................................................................... 63

2.3.3.1 Primary Endpoint ...................................................................................................... 63

2.3.3.2 Secondary Endpoints ................................................................................................... 64

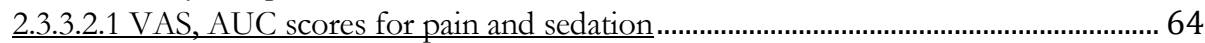

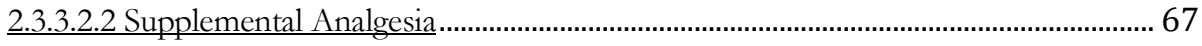

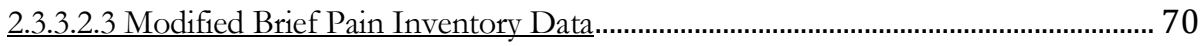

2.3.3.2.4 Side effects and satisfaction................................................................................. 71

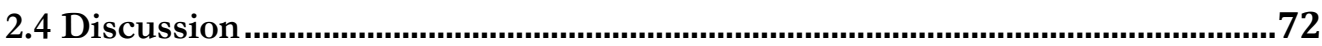

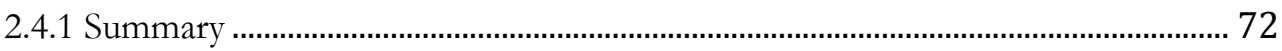

2.4.2 Oral analgesia post-caesarean delivery …………………………………………... 72

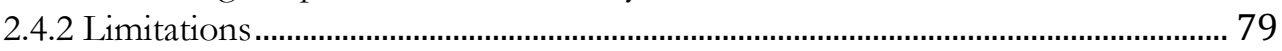

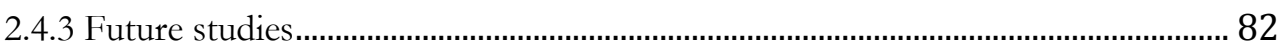

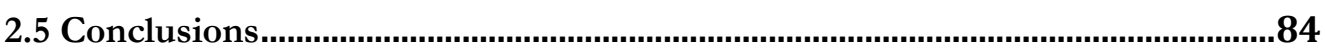

Appendix 1: Copy of Ethical Approval....................................................... 85

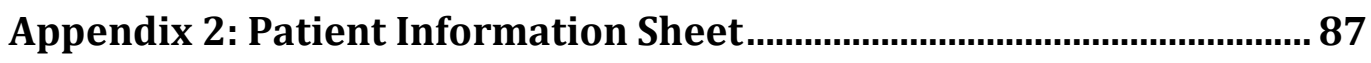

Appendix 3: Abstract of presentation at the 2008 NZSA/ASA Scientific Congress, Wellington, New Zealand.................................................... 90

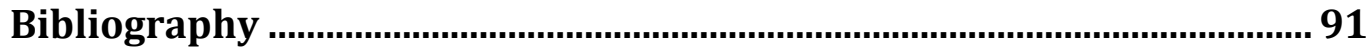




\section{Table of Figures}

FIGURE 1: PATIENT CONTROLLED MORPHINE USE WITH INCREASING DOSES OF INTRATHECAL MORPHINE 22 FIGURE 2: RELATIONSHIP OF INTRATHECAL MORPHINE DOSE AND PRURITUS.................................................. 23

FIGURE 3: PATIENT CONTROLLED MORPHINE USE WITH INCREASING DOSE OF EPIDURAL MORPHINE ......... 25

FIGURE 4: PREDICTED EPIDURAL MORPHINE DOSE RESPONSE CURVE ......................................................... 26

FIGURE 5: COMPARISON OF PRURITUS, PONV AND RESCUE ANALGESIA WITH EPIDURAL AND INTRATHECAL

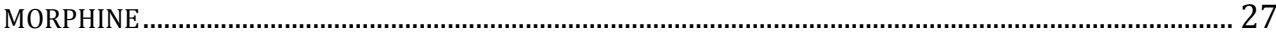

FIGURE 6: VAS SCORES WITH EPIDURAL AND INTRATHECAL MORPHINE .................................................... 28

FIGURE 7: EPIDURAL VERSUS INTRAVENOUS PETHIDINE AND VAS PAIN SCORES............................................ 30

FIGURE 8: DEPODUR LIPOFOAM PARTICLES …........................................................................................ 33

FIGURE 9: SUPPLEMENTAL ANALGESIA WITH EXTENDED RELEASE EPIDURAL MORPHINE AND

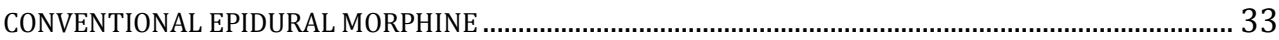

FIGURE 10: DURATION OF ANALGESIA WITH INTRATHECAL MORPHINE AND CLONIDINE COMBINATIONS... 35

FIGURE 11: ANATOMICAL DIAGRAM OF THE ABDOMINAL LAYERS AND THE TAP BLOCK.............................. 41

FIGURE 12: TIME TO FIRST ANALGESIC REQUEST IN TAP BLOCK VERSUS CONTROL SUBJECTS ...................... 42

FIGURE 13: MORPHINE CONSUMPTION POST-CAESAREAN WITH THE TAP BLOCK VERSUS CONTROL SUBJECTS

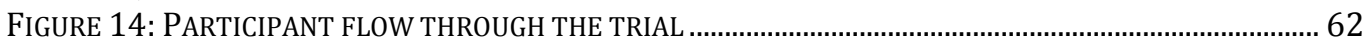

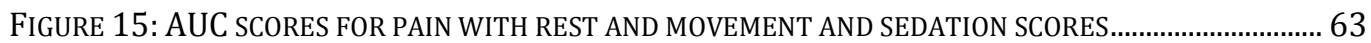

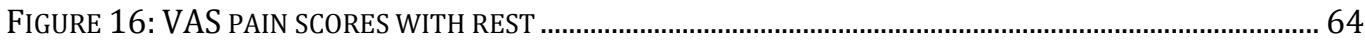

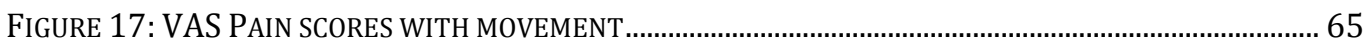

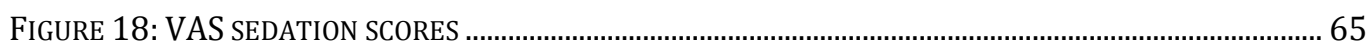

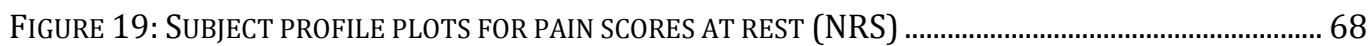

FIGURE 20: SUBJECT PROFILE PLOTS FOR PAIN SCORES WITH MOVEMENT (NRS) .......................................... 69 
Table of Tables

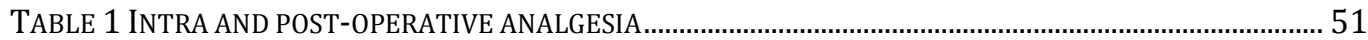

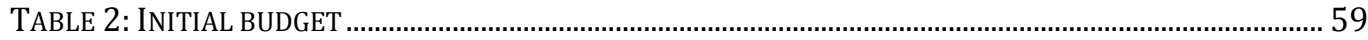

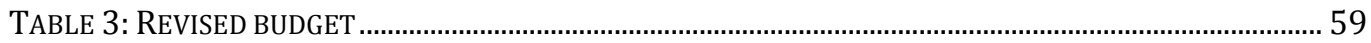

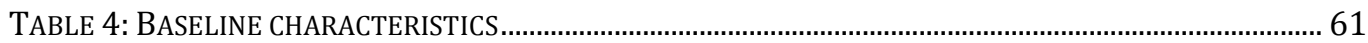

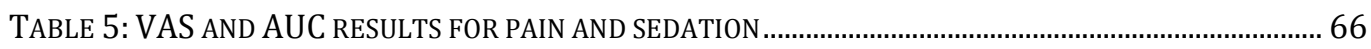

TABLE 6: MODIFIED BRIEF PAIN INVENTORY DATA .................................................................................... 70

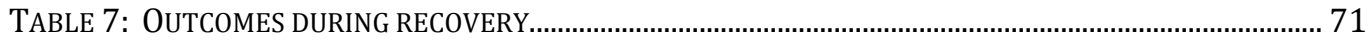




\section{Acknowledgements}

First and foremost I would like to thank my wonderful family starting with my wife Vini, who, without her support, encouragement and love, none of this would have been possible. She has been incredibly tolerant of all my endeavours over the past 3 years and has allowed me the freedom to pursue a busy research and clinical career, as well as being an amazing mother to our two children. To my parents, John and Margaret I also owe a great deal of thanks for all their continued support and for the sacrifices that they made to lay the foundations for my education from such a young age.

Secondly, I would like to extend my thanks to Professor Michael Paech who as well as being my primary supervisor, has also been a mentor over the last four years. It has been a privilege to work with such a highly regarded clinician and academic, and one who is also incredibly humble. His guidance, enthusiasm and tolerance of my questions is greatly appreciated.

I would like to thank my Head of Department, Dr Tim Pavy, for his continued support and encouragement since I started at King Edward Memorial Hospital for Women. He has been incredibly flexible with my rostering so that I could attend the various lectures and exams associated with this Masters, as well as being a mountain of support when times have been tough. In this same regard I would also like to thank Professor Gerald Watts who has also been a supervisor and a source of continual encouragement and support. He has always been approachable and happy to answer any question, no matter how minor.

This research project would not have been possible without the work of our two dedicated research midwives, Desiree Cavill and Tracy Bingham. They have worked tirelessly to ensure that the data collection was accurate and that the post natal staff as well as the patients had a clear understanding of the trial and what it entailed. In this same regard, the nursing staff of our recovery unit and the post natal staff were also essential to the successful completion of this project and I am grateful for the support that they have provided. 
Thanks must also go to the Pharmacy Department at King Edward Memorial Hospital for their support in ensuring that the trial was able to run as a true double blind study. They made the setup of the study very simple by taking on the whole issue of preparing a sterile intrathecal morphine solution as well as coming up with the dummy oxycodone medication.

The statistical support from the School of Women's and Infants' Health has been fantastic. Dr Dorota Doherty assisted with the initial design and planning of the study whilst Elizabeth Nathan has done the actual analysis and spent endless hours explaining findings and redoing data analysis for me.

I would also like to thank the University of Western Australia for providing this degree course. I have learnt so much over the duration of the course and have been able to meet and network with some of the top researchers in Western Australia. The teaching that I have received has empowered me to feel confident in my knowledge of trial design and statistical methods, two previous weak areas for myself. I now feel a significant advantage for future research activities with the knowledge obtained from this degree.

I would also like to acknowledge Dr Martin Schuitemaker who introduced me to the concept of oral analgesia for post-caesarean analgesia in New Zealand. It was his ideas which were the motivation for me to conduct this clinical research project.

Finally, I must extend my thanks to the Australian and New Zealand College of Anaesthetists for their generous financial support for this study. Without their funding we would have struggled to be able to perform this research. 


\section{Publications, Abstracts and Presentations Arising from this Thesis}

\section{Publications}

McDonnell NJ, Keating ML, Muchatuta NA, Pavy TJG, Paech MJ. Analgesia after caesarean delivery. Anaesthesia and Intensive Care, Vol 37, No.4, July 2009. Pg 539551

Mcdonnell NJ, Paech MJ, Browning RM, Nathan EA. A randomised comparison of regular oral oxycodone and intrathecal morphine for post-caesarean analgesia. International Journal of Obstetric Anesthesia (2009), doi:10.1016/j.ijoa.2009.03.004

\section{Published Abstracts}

Oral oxycodone versus intrathecal morphine for post-caesarean analgesia. $\mathrm{N}$ McDonnell, M Paech, D Doherty. Published in Anaesthesia and Intensive Care, Vol 36, No.6, November 2008, pg 885.

\section{Presentations}

Post-caesarean Analgesia: Getting it right. NJ McDonnell. Rural Anaesthesia Special Interest Group Conference, Hunter Valley, July 2009

Oral oxycodone versus intrathecal morphine for post-caesarean analgesia. $\underline{\mathrm{N}}$ McDonnell, M Paech, D Doherty. Presented at the Australian Society of Anaesthetists and New Zealand Society of Anaesthetists Combined Scientific Congress, October 11-14, Wellington, New Zealand.

Oral oxycodone versus intrathecal morphine for post-caesarean analgesia. $\mathrm{N}$ McDonnell, M Paech, E Nathan, R Browning, A Sinha. Presented at the Western Australian Winter Scientific Meeting (A Sinha presenting), June 2009, Perth, Australia 


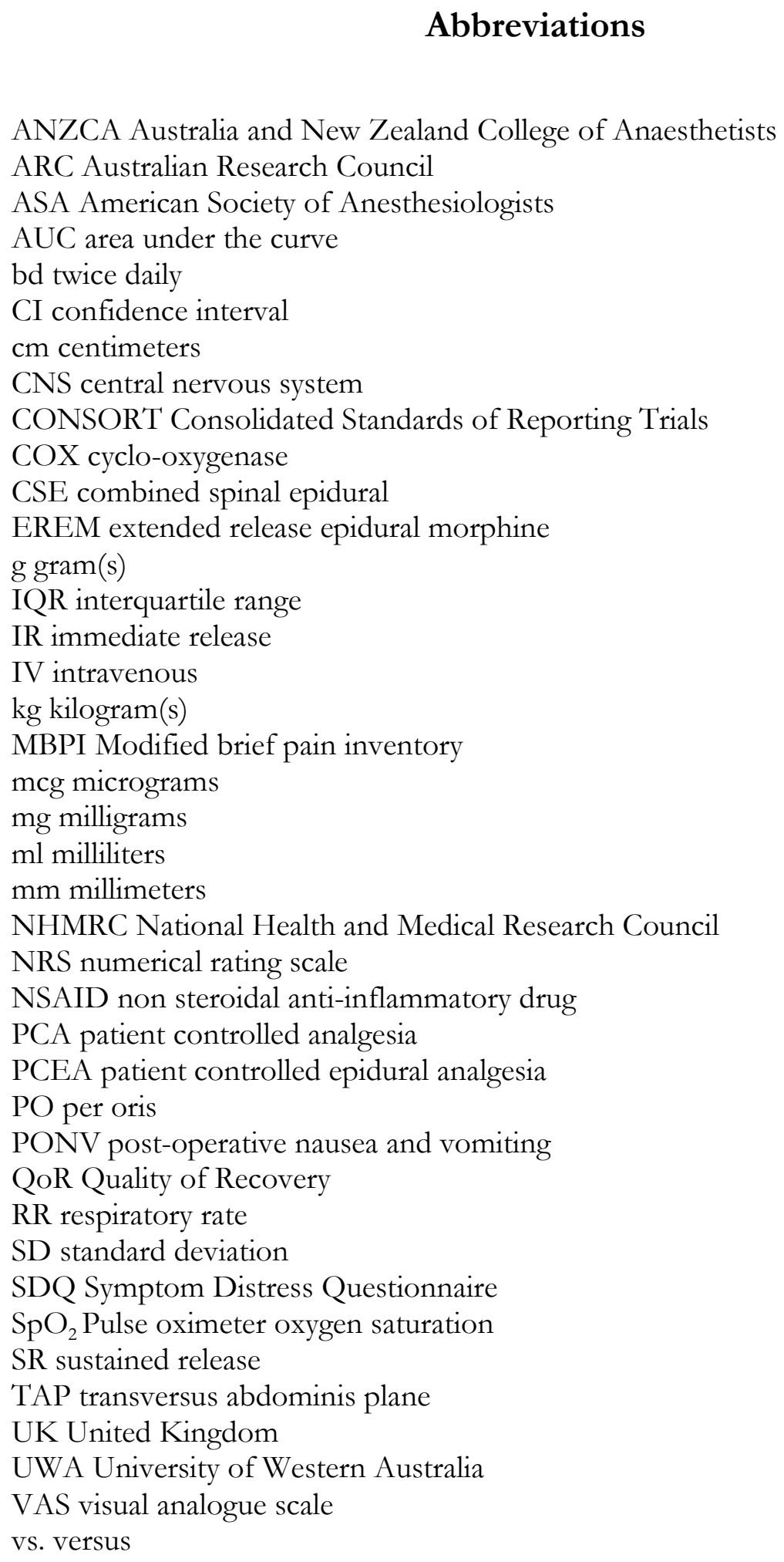

ANZCA Australia and New Zealand College of Anaesthetists ARC Australian Research Council ASA American Society of Anesthesiologists AUC area under the curve bd twice daily CI confidence interval $\mathrm{cm}$ centimeters CNS central nervous system CONSORT Consolidated Standards of Reporting Trials COX cyclo-oxygenase

CSE combined spinal epidural

EREM extended release epidural morphine $\operatorname{g~gram}(\mathrm{s})$

IQR interquartile range

IR immediate release

IV intravenous

kg kilogram(s)

MBPI Modified brief pain inventory mcg micrograms

mg milligrams $\mathrm{ml}$ milliliters mm millimeters

NHMRC National Health and Medical Research Council NRS numerical rating scale NSAID non steroidal anti-inflammatory drug PCA patient controlled analgesia

PCEA patient controlled epidural analgesia PO per oris PONV post-operative nausea and vomiting QoR Quality of Recovery

$\mathrm{RR}$ respiratory rate

SD standard deviation

SDQ Symptom Distress Questionnaire $\mathrm{SpO}_{2}$ Pulse oximeter oxygen saturation SR sustained release

TAP transversus abdominis plane

UK United Kingdom

UWA University of Western Australia

VAS visual analogue scale

vs. versus 


\section{Statement of candidate contribution}

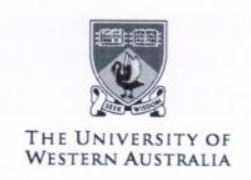

DECLARATION FOR THESES CONTAINING PUBLISHED WORK AND/OR WORK PREPARED FOR PUBLICATION

The examination of the thesis is an examination of the work of the student. The work must have been substantially conducted by the student during enrolment in the degree.

Where the thesis includes work to which others have contributed, the thesis must include a statement that makes the student's contribution clear to the examiners. This may be in the form of a description of the precise contribution of the student to the work presented for examination and/or a statement of the percentage of the work that was done by the student.

In addition, in the case of co-authored publications included in the thesis, each author must give their signed permission for the work to be included. If signatures from all the authors cannot be obtained, the statement detailing the student's contribution to the work must be signed by the coordinating supervisor.

Please sign one of the statements below.

1. This thesis does not contain work that I have published, nor work under review for publication.

Student Signature

2. This thesis contains only sole-authored work, some of which has been published and/or prepared for publication under sole authorship. The bibliographical details of the work and where it appears in the thesis are outlined below.

Student Signature

3. This thesis contains published work and/or work prepared for publication, some of which has been coauthored. The bibliographical details of the work and where it appears in the thesis are outlined below. The student must attach to this declaration a statement for each publication that clarifies the contribution of the student to the work. This may be in the form of a description of the precise contributions of the student to the published work and/or a statement of percent contribution by the student. This statement must be signed by all authors. If signatures from all the authors cannot be obtained, the statement detailing the student's contribution to the published work must be signed by the coordinating supervisor.

This thesis is based upon two papers which have been co-authored and published. The author of this thesis was also the first author on both papers that have been published. Details of the contribution by the various authors of each paper is given in a separate declaration and copies of each publication are also provided in the Appendices.

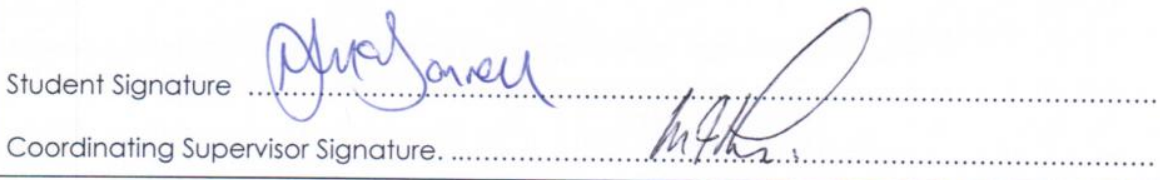




\section{Candidate contribution to the following published manuscripts:}

1. McDonnell NJ, Keating ML, Muchatuta NA, Pavy TJG, Paech MJ. Analgesia after caesarean delivery. Anaesthesia and Intensive Care, Vol 37, No.4, July 2009. Pg $539-551$

The candidate was the primary author for the above review article and was responsible for writing over $90 \%$ of the review and for the submission and revision process. The co-authors were responsible for the background reading and literature search in several areas of their own expertise, whilst Prof. Paech was responsible for the overall supervision of the article and the proof reading and editing of the various versions.

2. Mcdonnell NJ, Paech MJ, Browning RM, Nathan EA. A randomised comparison of regular oral oxycodone and intrathecal morphine for post-caesarean analgesia. International Journal of Obstetric Anesthesia (2009), doi:10.1016/j.ijoa.2009.03.004

The candidate was the primary author for the above article and was responsible for writing approximately $80-90 \%$ of the manuscript, as well as the submission and revision process. RM Browning was involved in aspects of the trial but did not contribute to the manuscript and EA Nathan did our final statistical work. Prof. Paech assisted with the study design, supervised the writing of the manuscript and contributed significantly to aspects of the discussion that the author had difficulty with.

\section{Candidate contribution to the study design and funding application:}

The candidate was responsible for the original concept of the study on which this thesis is based. This concept was then developed in conjunction with Prof. Paech into a trial protocol. Funding options were considered, as funding for research of this nature is limited it was decided to apply through ANZCA for a funding grant under the Lennard Travers Professorship. This is a prestigious award which is only granted every four years. Given the candidates lack of a suitable track record at the time of applying for funding it was felt that to optimize the chances of success Prof. Paech should be listed as the Principal Investigator and the candidate as a CoInvestigator. The candidate was actively involved at all stages of the funding application and the response to the ANZCA reviewers for this application. 


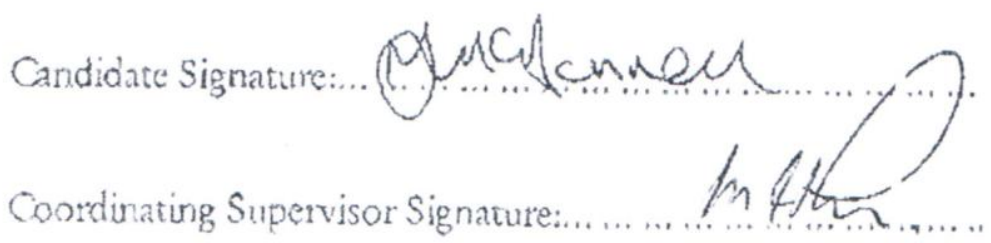




\section{Chapter One: Literature Review}

\subsection{Introduction}

Over the last two decades the number of caesareans being performed has increased dramatically (Villar, Valladares et al. 2006). High quality post-operative analgesia is important because the new mother has to recover from major intra abdominal surgery whilst also caring for her newborn baby (Lavand'homme 2006; Pan 2006). Many options are available but tailoring the method to the individual can be problematic because it has been difficult to predict the severity of post-operative pain or the individual response to a regimen.

A number of factors can influence the choice of analgesic regimen in any given hospital. These include the patient preferences and expectations, the expected surgical difficulty and duration, and the preferences and experience of the anaesthetist. In addition, a caesarean may be performed under spinal, epidural or general anaesthesia such that some methods of pain relief are not universally suitable. A hospital may be unable to provide certain methods of analgesia because of issues related to staff education, training, workload or drug availability. Some techniques may be contraindicated in certain obstetric circumstances such as patient refusal, pre eclampsia, bleeding diathesis and local infection. Consequently, the number of post-caesarean analgesic options continues to expand whilst existing methods are refined.

Opioids, administered by the intravenous or neuraxial route, form the foundation of most multimodal analgesic regimens after caesarean delivery (Gadsden, Hart et al. 2005). This literature review examines the current evidence supporting the commonly employed methods of post-caesarean analgesia and the gaps in the knowledge in regard to oral opioid administration in this setting. It commences with a brief overview of post-caesarean pain, followed by a review of the current literature on oral opioids post-caesarean delivery. It then examines neuraxial techniques for post-caesarean analgesia, followed by a discussion of intravenous opioids and oral adjunct analgesic drugs, and local anaesthetic based nerve block techniques. It ends with an examination of the issue of chronic post-caesarean pain. 


\subsection{Post-caesarean pain}

Post-caesarean pain is likely to have at least two components (Lavand'homme 2006), with pain arising from both the abdominal and uterine incisions, with a third potential source being pain associated with uterine involution post-delivery (Pavy, Gambling et al. 1995). Somatic pain arising from nociceptors within the abdominal wound has both cutaneous and deep components. It is transmitted within the anterior divisions of the spinal segmental nerves, usually T10-L1, which run laterally in the abdominal wall between the layers of the transversus abdominis and internal oblique muscles (McDonnell, O'Donnell et al. 2007). Visceral uterine nociceptive stimuli return via afferent nerve fibres that ascend through the inferior hypogastric plexus and enter the spinal cord via the T10-L1 spinal nerves (Moore 1992).

Traditionally it has been difficult to predict the severity of post-caesarean pain and analgesic needs because of large interpatient variability in the intensity of pain experienced, as well as difficulties in predicting the response to an individual analgesic regimen. Factors that have been associated with significant post-operative pain have included the duration of surgery, probably as a consequence of more extensive dissection, and a lower dermatomal level of sensory anaesthesia at the time of incision, which may contribute to greater nociceptive input to the spinal cord and enhanced central sensitization (Eisenach, Coghill et al. 2005). A series of pre-operative physical (thermal pain threshold) and psychological tests have been shown to predict the upper $20^{\text {th }}$ percentile of post-caesarean pain scores with a sensitivity of between $0.71-0.80$ and a specificity of 0.76 to 0.80 , and to show improved prediction over single test models (Granot, Lowenstein et al. 2003; Pan, Coghill et al. 2006).

An ideal post-caesarean analgesic regimen would be one that was cost effective, simple to implement and with minimal impact on staff workload. It would provide consistent and high quality pain relief whilst catering for wide interpatient variability yet have a low incidence of side effects and complications. It would not interfere with the maternal care of the newborn or with the establishment of breast feeding and there would be minimal drug transfer into breast milk and no adverse effects on the newborn. In this regard, a multimodal approach based on opioids has been commonly recommended (Lavand'homme 2006; Pan 2006). 


\subsection{Oral opioids}

Oral opioids have traditionally been used as 'step-down' analgesics following primary management with neuraxial or intravenous opioids in the post-caesarean setting. The advantages of the oral route include simplicity, convenience and familiarity for both patients and staff and potentially fewer side effects compared with the intravenous or neuraxial route.

The consideration of a primarily oral analgesic regimen after caesarean was first noted in the obstetric literature in 1998 by Monagle et al. They published audit data from 20 patients given a regimen of oral morphine, paracetamol and aspirin postcaesarean (Monagle, Molnar et al. 1998). They reported a low incidence of side effects and high levels of satisfaction with this technique. In the year 2000, Jakobi et al expressed concern in regards to the expensive nature of traditional methods of post-caesarean analgesia, as well as the need for specialised personnel and training (Jakobi, Weiner et al. 2000). They conducted an open, prospective study that compared oral immediate-release morphine sulphate $(30 \mathrm{mg})$ with dipyrone, a potent non steroidal anti-inflammatory agent. In the first arm of the study, subjects received $1 \mathrm{~g}$ dipyrone every 4 hours, with supplemental immediate release morphine sulphate $(30 \mathrm{mg})$ given if required. In the second arm of the study, morphine sulphate was given every 4 hours and dipyrone was used as the supplemental agent. Patients were allowed to freely withdraw from the study and cross over to the alternative protocol after the first supplemental dose was required. They found that $20 \%$ of patients in the regular morphine group changed protocols during the study compared to none in the dipyrone group, that pain scores overall were similar between groups and that maternal satisfaction was high in both groups.

The design of these studies unfortunately limits the conclusions that can be drawn but it did demonstrate that an oral regimen was potentially feasible post-caesarean. It also highlights the important role of non-steroidal anti-inflammatory drugs (NSAIDs) for post-caesarean analgesia and the high number of women crossing over to the NSAID group emphasizes that opioids are not necessarily the ideal drugs to control all the potential pain mechanisms activated after caesarean surgery. 
Also in the year 2000, Holt published some audit data on the results of their experience using patient controlled Oramorph (oral morphine) (Holt 2000). Whilst not providing details of the actual regimen in use, high levels of maternal satisfaction were demonstrated. In response to this, Antrobus, in the same journal in early 2001, described their use of Oramorph. They had been using Oramorph, in combination with an NSAID and paracetamol, since 1996 with success and recommended that studies that examine post-caesarean analgesia should also focus on maternal function, not just analgesia (Antrobus 2001). This led to another UK institution establishing an on-demand oral opioid regimen using Oramorph, with mothers being allowed to access the drug from locked, secure cupboards in their own rooms when required (with a considerable number of administrative obstacles to negotiate prior to implementation) (Scott, Cameron et al. 2001). Whilst these authors mention that they were in the process of conducting a post implementation audit, the results of that audit have never been published.

In 2002 Jakobi et al then published the results of a follow-on study to their original study from 2000 (Jakobi, Solt et al. 2002). Again this study was a prospective, non randomised open study. On this occasion they compared ibuprofen with oral morphine as the primary technique for post-caesarean analgesia and administered it at either a fixed dosing interval or on patient demand. It is once again difficult to draw many meaningful conclusions because of the open study design, but of interest was the finding that women in the fixed dosing interval group had higher overall satisfaction as well as lower overall pain scores. This highlighted the importance of providing post-operative analgesia regularly, rather than in an on-demand basis.

Since the 2002 study by Jakobi, there has only been one other study published in regards to oral analgesia post-caesarean. In 2006 Davis et al published the results of their study in which women were randomised to either oral analgesia with an oxycodone-paracetamol combination or an intravenous morphine patientcontrolled analgesia (PCA) technique in combination with regular intravenous ketorolac (Davis, Esposito et al. 2006). This study also has significant limitations. Women were not blinded to the intervention they received. Analgesia was assessed at the 6 and 24 hour post-operative mark using a 0-10 visual analogue scale. No mention was made of whether dynamic or rest pain was assessed, and only one pain score was reported. The investigators found less pain in the oral oxycodone- 
paracetamol group who also reported less nausea and sedation with a trend towards decreased pruritus.

The study by Davis et al presents the most robust data to date on the potential benefits of an oral opioid regimen, but still left a number of questions unanswered, and the lack of blinding as well as the lack of a distinction between rest and dynamic pain (which is of greater functional and clinical importance) greatly limits the conclusions that can be drawn from this study.

The other oral opioid that has received some attention in the obstetric anaesthesia literature is tramadol, an analgesic with weak mu-opioid receptor agonism and activity at noradrenergic, serotonergic and GABAergic systems (Duthie 1998). It appears to be commonly used as a rescue analgesic, as well as a step down agent. Despite being used in breast feeding mothers for a considerable period of time, data on the breast milk transfer for both oxycodone and tramadol had been limited until recent research showed that both oxycodone and tramadol transfer into breast milk is below that considered clinically significant for the neonate (Seaton, Reeves et al. 2007; Ilett, Paech et al. 2008).

\subsection{Neuraxial techniques for post-caesarean analgesia}

Neuraxial (spinal or epidural) anaesthesia has well documented benefits over general anaesthesia for caesarean delivery, particularly with respect to maternal safety (Hawkins, Koonin et al. 1997). These techniques also provide the anaesthetist with an effective and convenient route of opioid administration. Neuraxial opioids differ primarily in their potency, onset, duration of action and side effects (Chaney 1995). In addition, a limited number of other analgesic drugs may also be utilised through intrathecal or epidural administration.

\subsubsection{Spinal/Intrathecal opioids}

Opioids, especially morphine, are central to many intrathecal based analgesic regimens and act principally on mu-opioid receptors in the substantia gelatinosa of the dorsal horn. Whilst intrathecal fentanyl and sufentanil are both widely given for their intraoperative analgesic effect, unless used in high doses (eg fentanyl 40-60 mcg) their effects are too short-lived to be of benefit post-operatively and they do not alter 24 hour opioid consumption (Belzarena 1992; Dahlgren, Hultstrand et al. 
1997). Their short analgesic duration of action contrasts with the long duration from neuraxial morphine (Chaney 1995). This is due to the latter's low lipid solubility, such that it takes longer to be removed from neural tissues. The comparatively lower lipid solubility of morphine delays its onset of action but prolongs its duration of action (Dahl, Jeppesen et al. 1999). Moreover, the longer residence time of morphine in the cerebrospinal fluid allows it to spread rostrally, from which complications such as respiratory depression may arise (Bromage, Camporesi et al. 1982; Chaney 1995).

\subsubsection{Intrathecal morphine}

Many doses of intrathecal morphine have been investigated and at doses above 100 mcg no clear dose-response relationship has been demonstrated. Palmer et al studied doses between 0 and 500 mcg in 108 women undergoing elective caesarean delivery and found a ceiling analgesic effect, as measured by patient-controlled intravenous morphine use, with doses of morphine above $75 \mathrm{mcg}$ (Figure 1) (Palmer, Emerson et al. 1999). Higher doses conferred no additional analgesic benefit but caused a dose dependent increase in side effects, particularly pruritus (Figure 2). Palmer et al also noted that despite high doses of intrathecal morphine, most parturients continued to administer additional opioid analgesia at a low but constant rate. This may be explained by an interaction between spinal and supraspinal opioid receptors and sites of action, such that both may need to be occupied for optimal analgesia (Palmer, Emerson et al. 1999; Palmer, Nogami et al. 2000). Other studies support a dose of no more than $100 \mathrm{mcg}$ as being optimal, and smaller doses $(25-50 \mathrm{mcg})$ when combined with a systemic NSAID can be effective (Cardoso, Carvalho et al. 1998). Nevertheless, a dose of $100 \mathrm{mcg}$ of intrathecal morphine is unsatisfactory in a small but significant percentage of women. Swart et al demonstrated that after intrathecal morphine 100 mcg most parturients use less than $10 \mathrm{mg}$ of intravenous morphine in the first 24 hours, but $10 \%$ used more than $40 \mathrm{mg}$ (Swart, Sewell et al. 1997). This suggests that in their study, although intrathecal morphine was effective in the majority of women in this study, in the $10 \%$ of women requiring more than $40 \mathrm{mg}$ of supplemental morphine it is best described as ineffective.

Intrathecal and epidural morphine share a similar side effect profile, with pruritus the most common side effect and the other side effects being nausea, vomiting, reactivation of oral herpes simplex, urinary retention and delayed respiratory 
depression (Chaney 1995). From intrathecal morphine 50-250 mcg the number needed to harm one individual (post-caesarean delivery) is 2.6 (95\% CI 2.1-3.3) for pruritus, 6.3 (95\% CI 4.2-12.5) for nausea and 10.1 (95\% CI 5.7-41.0) for vomiting (Dahl, Jeppesen et al. 1999). If a $100 \mathrm{mcg}$ dose is used, it is estimated that $43 \%$ of women will experience pruritus, 12\% vomiting and 10\% nausea (Dahl, Jeppesen et al. 1999). Neuraxial morphine administration has also been linked to the reactivation of oral herpes simplex. In a study of women with a past history of oral herpes simplex, reactivation occurred in 38\% receiving intrathecal morphine compared with $16 \%$ of those receiving intravenous morphine (Davies, Vallejo et al. 2005).

Respiratory depression is an uncommon and potentially serious side effect but the incidence in the obstetric population is difficult to determine. Pooled data from a meta-analysis that included 485 caesarean patients showed only one case of respiratory depression, as defined by a respiratory rate of less than 10 breaths per minute (Dahl, Jeppesen et al. 1999). Abouleish et al studied 856 parturients who received $200 \mathrm{mcg}$ of intrathecal morphine at caesarean and found respiratory depression, as defined by a $\mathrm{SpO} 2<85 \%$ or a respiratory rate of $<10$ breaths per minute, in 8 patients $(0.93 \%$ ), all of whom were obese (Abouleish, Rawal et al. 1991). More recently, Kato et al reviewed 1915 women who had received 150 mcg of intrathecal morphine for post-caesarean analgesia and documented 4 patients with mild respiratory depression and only 1 patient who required naloxone (Kato, Shimamoto et al. 2008). The physiological changes of pregnancy, specifically the higher respiratory rate associated with elevated progesterone levels, as well as the younger age and relative lack of co-morbidities may provide a greater margin of safety in comparison to other patient populations (Chaney 1995; Kato, Shimamoto et al. 2008). 
Figure 1: Patient controlled morphine use with increasing doses of intrathecal morphine

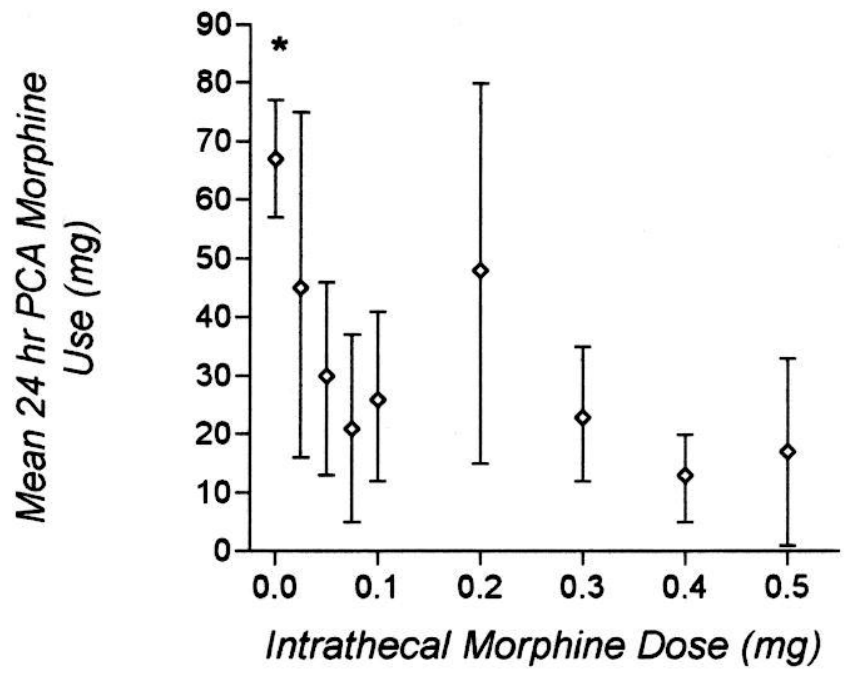

Mean ( $\pm 95 \%$ CI $)$ 24-h patient-controlled morphine use with increasing doses of intrathecal morphine demonstrating continued use of IV morphine even at high doses of intrathecal morphine. ${ }^{*} \mathrm{p}<0.05$.

From: Palmer et al. Anesthesiology 1999;90:437-444 
Figure 2: Relationship of intrathecal morphine dose and pruritus

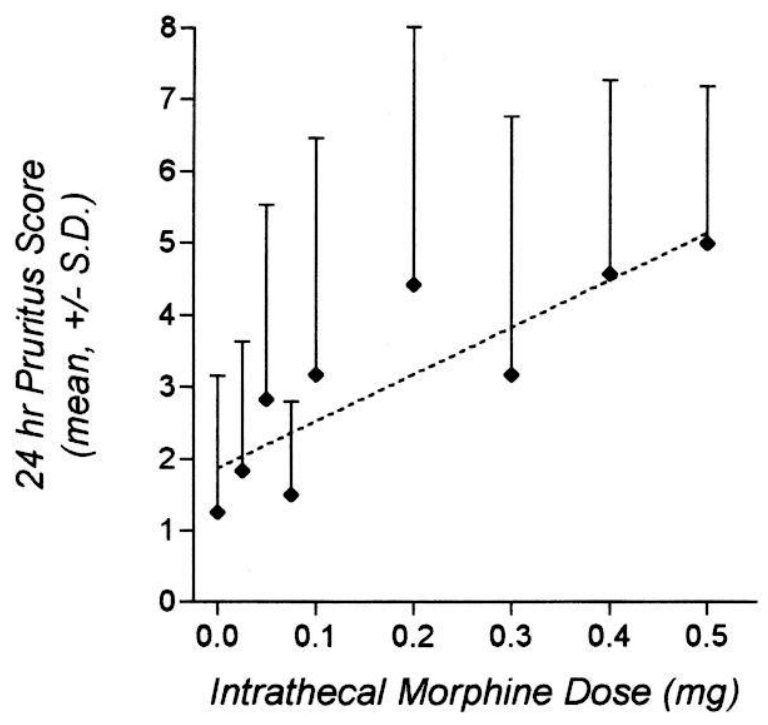

Mean 24 hour pruritus scores (mean \pm SD) demonstrating increased incidence of pruritus with increasing dose of intrathecal morphine. Scores are a cumulative score over 24 hours (scored none $=0$, mild $=1$, moderate $/$ severe $=2$; total out of 21). From: Palmer et al. Anesthesiology 1999;90:437-444

\subsubsection{Intrathecal diamorphine}

Diamorphine, in a dose of between $250-375 \mathrm{mcg}$, is a suitable alternative to intrathecal morphine (Kelly, Carabine et al. 1998; Lane, Evans et al. 2005; Wrench, Sanghera et al. 2007). It is particularly popular in the United Kingdom, where it is more commonly used than intrathecal morphine for post-caesarean analgesia but it is not readily available in Australia (Giovannelli, Bedforth et al. 2008). Being more lipophilic, diamorphine has a faster onset of action and despite a short half life in cerebrospinal fluid, once it has diffused into neural tissues it is metabolised into its active components, 6-acetylmorphine and morphine, thus increasing its duration of action (Lane, Evans et al. 2005). Consequently diamorphine is attractive in providing not only intra-operative analgesia of similar quality to intrathecal fentanyl but also prolonged post-operative analgesia. Side effects are dose dependent, with pruritus and nausea common, occurring in $90 \%$ and $30-50 \%$ of women respectively after a $200 \mathrm{mcg}$ dose at caesarean delivery (Wrench, Sanghera et al. 2007). 


\subsubsection{Epidural opioids}

There are several approaches to epidural opioid delivery for post-caesarean analgesia. Morphine's low lipid solubility and prolonged duration of action means that a single bolus dose is often satisfactory for the first 24 hours (Chaney 1995). Fentanyl and pethidine are more lipid soluble and thus have a short duration of action, making them better suited to patient or nurse controlled techniques (Chaney 1995). The predominant site of action may vary with the administration route and dose and as previously discussed with intrathecal morphine, both spinal cord and supraspinal sites of action may coincide (Ginosar, Columb et al. 2003; Mather and Cousins 2003; George 2006).

\subsubsection{Epidural morphine}

Like intrathecal morphine, a single dose of epidural morphine appears to reach a ceiling analgesic effect. In terms of analgesic quality and supplemental 24 hour morphine consumption, epidural morphine $3 \mathrm{mg}$ appears equivalent to intrathecal morphine $100 \mathrm{mcg}$ and usually provides effective analgesia for 12-24 hours (Sarvela, Halonen et al. 2002). Palmer et al studied epidural morphine 0 to $5 \mathrm{mg}$ and found no difference in cumulative systemic morphine use for doses above $3.75 \mathrm{mg}$ (Figures 3 and 4) (Palmer, Nogami et al. 2000). Patients receiving the higher doses of epidural morphine continued to request additional opioid, lending further support to the theory of the importance of both spinal and supraspinal opioid receptor occupation.

There are few studies comparing epidural and intrathecal morphine. Sarvela et al compared epidural morphine $3 \mathrm{mg}$ with intrathecal morphine 100 or $200 \mathrm{mcg}$ (Sarvela, Halonen et al. 2002). Pain relief did not differ significantly between groups but rescue analgesia was requested more frequently in the $100 \mathrm{mcg}$ intrathecal morphine group (Figures 5 and 6) which experienced less pruritus (65\% vs. 91\% in the 100 mcg and 200 mcg groups respectively and $74 \%$ in the epidural group) (Figure 5). 
Figure 3: Patient controlled morphine use with increasing dose of epidural morphine

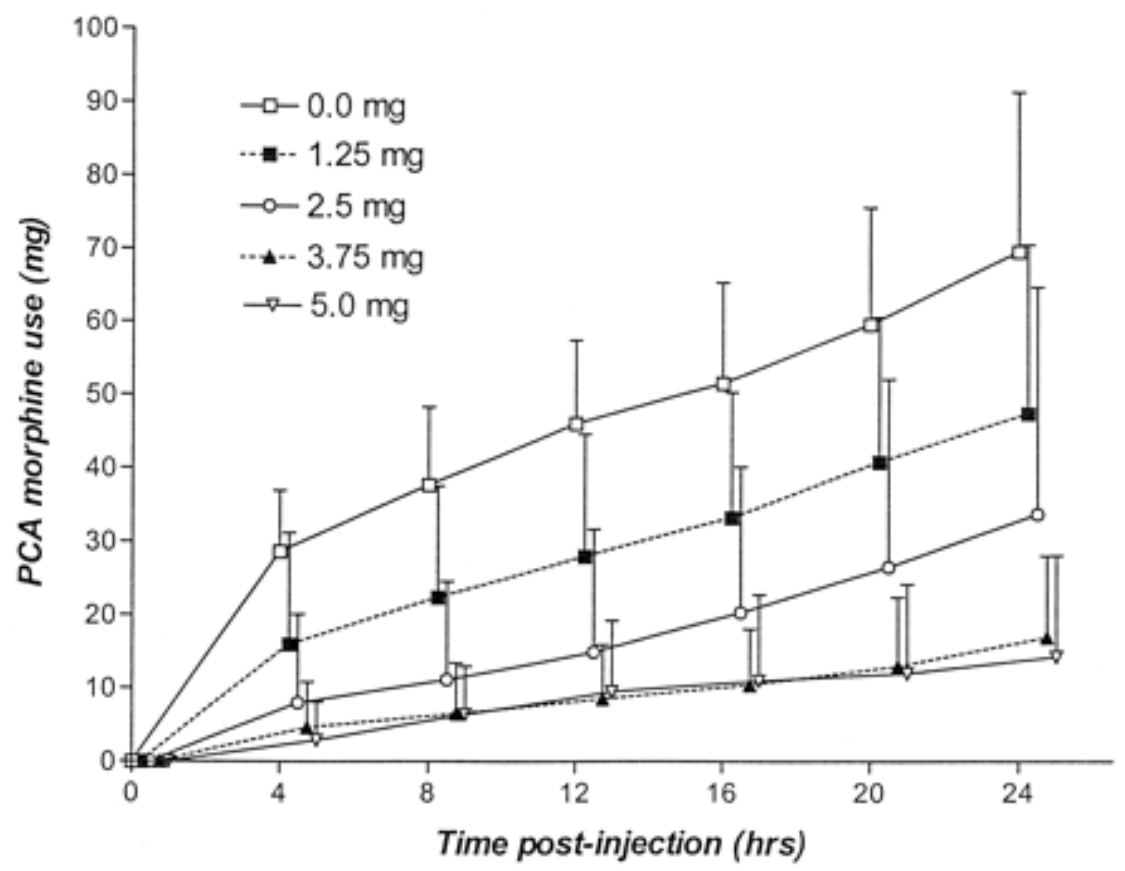

Cumulative patient controlled morphine use (mean and 95\% CI) over 24 hours with increasing doses of epidural morphine demonstrating a ceiling effect in analgesia with a dose greater than $3.75 \mathrm{mg}$.

From: Palmer, CM et al. Anesth Analg 2000;90:887-891 
Figure 4: Predicted epidural morphine dose response curve

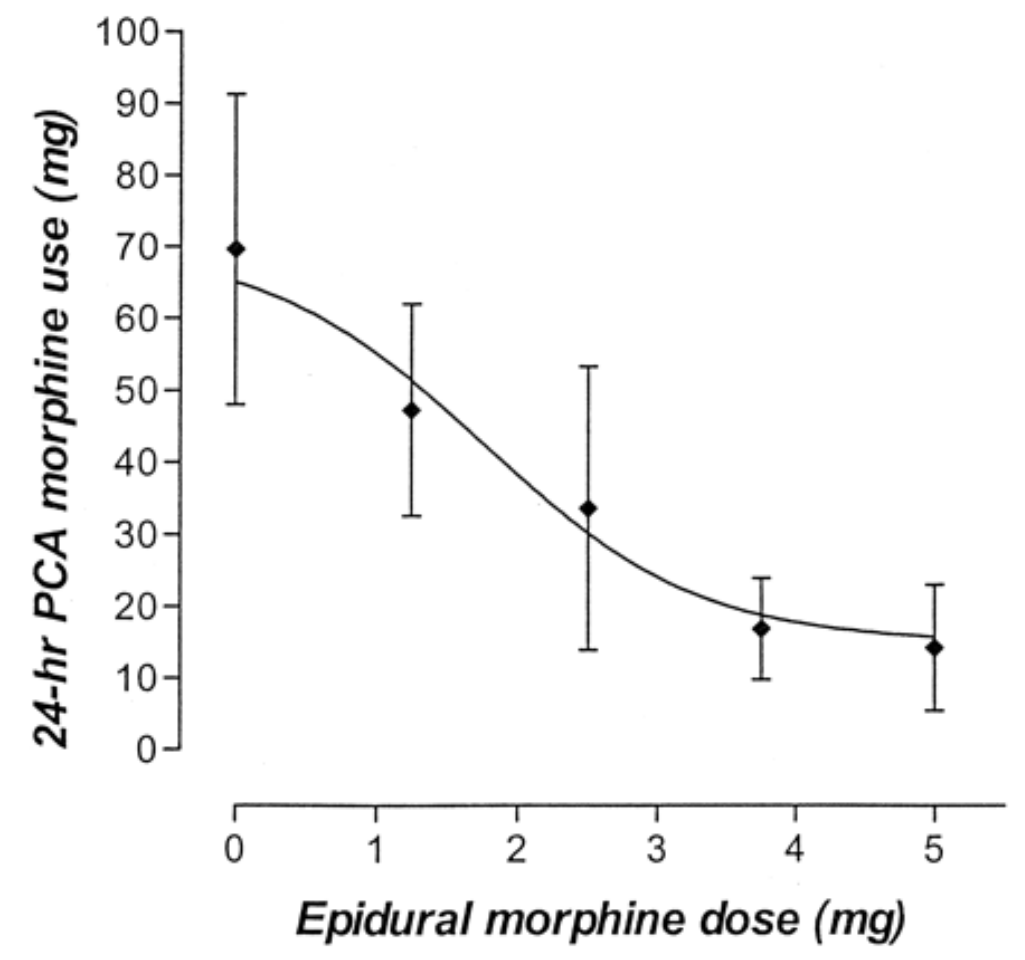

Dose response curve predicting patient controlled morphine use (mean $\pm 95 \% \mathrm{CI}$ ) after different doses of epidural morphine.

From: Palmer, CM et al. Anesth Analg 2000;90:887-891 
Figure 5: Comparison of pruritus, PONV and rescue analgesia with epidural and intrathecal morphine

Postoperative variables in the ward up to 24 hours

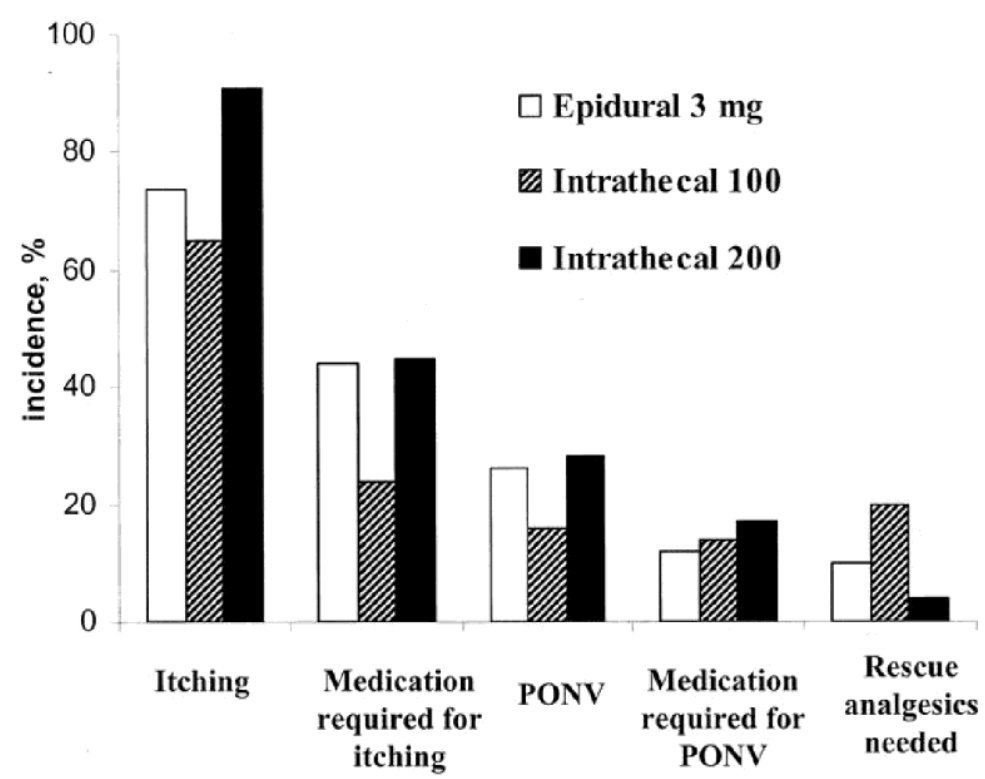

Percentage of patients with itching and PONV and those requesting treatment with epidural (3 mg) and intrathecal (100 and $200 \mathrm{mcg}$ ) morphine. Patients in the 100 mcg IT morphine group had less itching, required less interventions for itching but required more rescue analgesia.

From: Sarvela et al. Anesth Analg 2002;95:436440 
Figure 6: VAS scores with epidural and intrathecal morphine

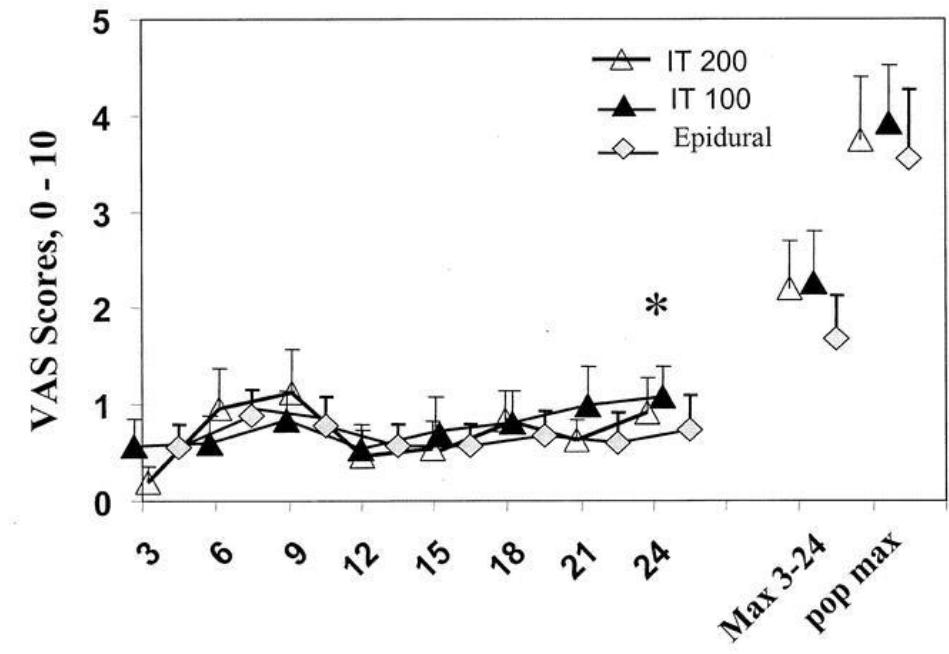

time, $\mathrm{h}$

Visual analog scale (VAS) scores of post-operative pain (mean and 95\% CI) during the first 24 hours with epidural versus intrathecal morphine. ${ }^{*} \mathrm{p}<0.05$.

From: Sarvela et al. Anesth Analg 2002;95:436-440 


\subsubsection{Epidural diamorphine}

Like intrathecal diamorphine, epidural diamorphine $2-3 \mathrm{mg}$ is popular for postcaesarean analgesia in the United Kingdom. Bloor et al audited 188 women receiving these doses and found that $92 \%$ had pain rated as mild or less (Bloor, Sinden et al. 1999). The same investigators compared epidural diamorphine $3 \mathrm{mg}$ to intrathecal diamorphine $300 \mathrm{mcg}$ and found similar quality analgesia but a higher incidence and severity of pruritus in the intrathecal group $33 \%$ moderate to severe pruritus versus 3\% in the epidural group) (Bloor, Thompson et al. 2000). Compared with intrathecal morphine (200 mcg), epidural diamorphine $3 \mathrm{mg}$ has a shorter duration of action (time to first supplementary analgesia request of $13.8 \pm 6.5 \mathrm{~h}$ vs. $22.3 \pm 12.0 \mathrm{~h}, \mathrm{p}=0.04)$ but a lower incidence of nausea and vomiting $(73 \%$ vs. $41 \%$, $\mathrm{p}=0.01)$ (Caranza, Jeyapalan et al. 1999).

\subsubsection{Epidural pethidine}

Patient controlled epidural (PCEA) pethidine is used in a number of countries with one validated regimen being a $20 \mathrm{mg}$ bolus and $15 \mathrm{~min}$ lockout administered via a lightweight, single-use device (Banks and Pavy 2001). Intrathecal morphine $200 \mathrm{mcg}$ results in lower pain scores in the first 12 post-operative hours but is associated with more pruritus $(33 \%$ vs. $5 \%$ with PCEA pethidine, $\mathrm{P}<0.001)$ and a higher incidence of nausea requiring treatment $(50 \%$ vs. $8 \%$ with PCEA pethidine, $\mathrm{P}<0.001$ ) (Paech, Pavy et al. 2000). When compared to intravenous pethidine in a double blind, crossover trial, epidural pethidine produced significantly lower pain scores $(\mathrm{P}=0.0001)$ (Figure 7$)$, lower sedation scores $(\mathrm{p}=0.0001)$ and improved patient satisfaction $(\mathrm{P}=0.0001)$, such that more than $90 \%$ of women preferred the epidural route of administration (Paech, Moore et al. 1994). 
Figure 7: Epidural versus intravenous pethidine and VAS pain scores

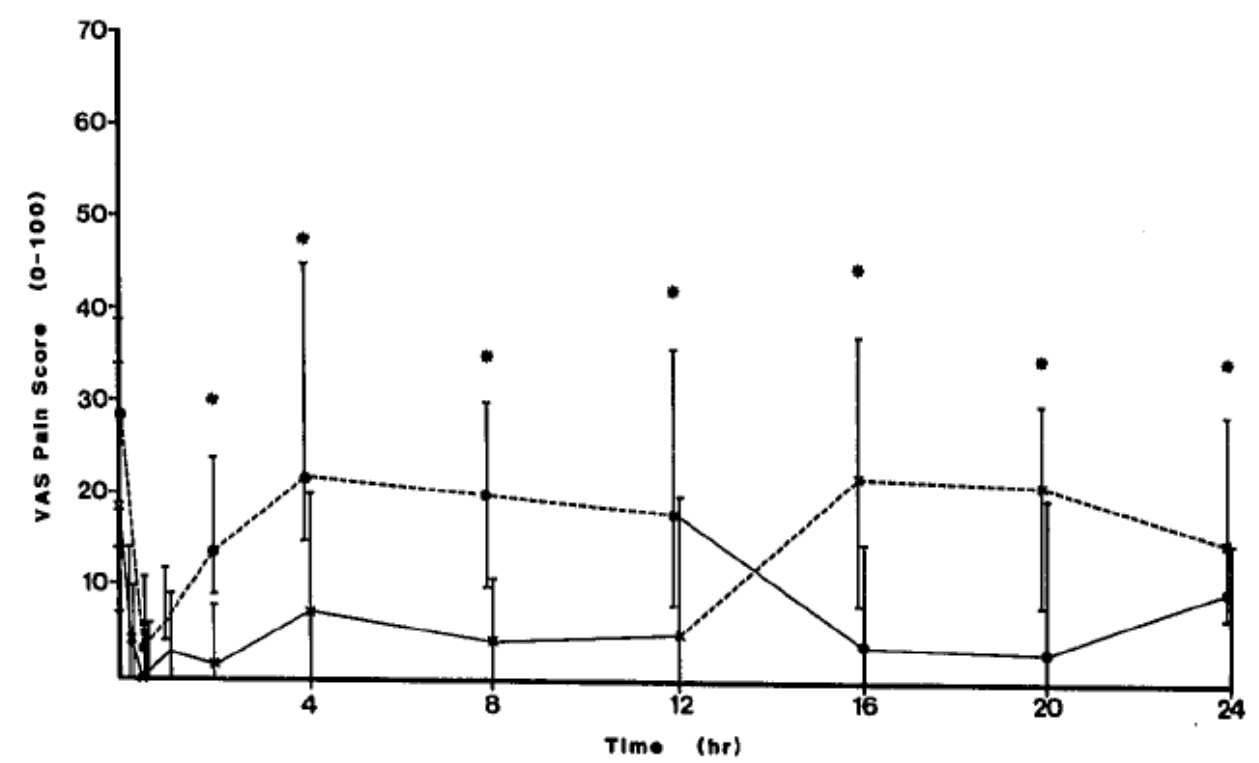

VAS pain scores (median and interquartile range) at rest during the first 24 hours post-caesarean comparing epidural (solid line) and intravenous (dashed line) patient controlled analgesia with pethidine. Pain scores are consistently lower when pethidine is administered epidurally. ${ }^{*} \mathrm{p}<0.05$.

From: Paech et al. Anesthesiology 1994;80:1268-1276 


\subsubsection{Epidural fentanyl}

Epidural fentanyl is widely used during labour and delivery and for non-obstetric post-operative analgesia but there is a paucity of published work describing its use for post-caesarean analgesia. When compared to epidural pethidine, Goh et al showed comparable patient-controlled analgesia but higher patient satisfaction and patient preference for epidural pethidine (Goh, Evans et al. 1996). Ngan Kee et al noted a similar patient preference for epidural pethidine rather than intravenous pethidine, although this was not the case for fentanyl (Ngan Kee, Lam et al. 1997). Cooper et al found improved analgesia from patient controlled epidural fentanyl compared with intravenous morphine (median pain scores with movement $31 \mathrm{~mm}$ [IQR 21-41] vs. $56 \mathrm{~mm}$ [30-71]), and less nausea and drowsiness (Cooper, Saleh et al. 1999).

The cumulative dose of epidural fentanyl can be reduced by the addition of bupivacaine or adrenaline (epinephrine) but this is of uncertain clinical benefit after caesarean delivery and introduces the possibility of local anaesthetic induced complications such as impaired ambulation and pressure sores. Cooper et al observed that the addition of bupivacaine $0.05 \%$ to epidural fentanyl $2 \mathrm{mcg} / \mathrm{ml}$ reduced fentanyl consumption by $57 \%$ but did not change patient satisfaction or lead to other clinical benefits (Cooper, Ryall et al. 1996). In contrast, Cohen et al showed that the addition of small amounts of bupivacaine $(0.01 \%)$ or epinephrine $(0.5 \mathrm{mcg} / \mathrm{ml})$ to epidural fentanyl not only decreased overall fentanyl consumption but also improved analgesia and satisfaction while reducing side effects (Cohen, Lowenwirt et al. 1998).

\subsubsection{Epidural sufentanil}

Epidural sufentanil has similar clinical characteristics to fentanyl in regards to onset and duration, with a relative analgesic potency compared with fentanyl of approximately 5:1 (Grass, Sakima et al. 1997). Cohen et al compared epidural sufentanil and fentanyl (with bupivacaine and epinephrine) by background infusion and patient demand bolus, reporting more vomiting (12\% vs. $4.8 \%)$ and dizziness/lightheadedness in the sufentanil group (Cohen, Amar et al. 1993). This, together with the lack of any clear advantages over other neuraxial opioids, has limited its popularity for post-caesarean analgesia. 


\subsubsection{Extended release epidural morphine}

A new formulation of morphine in which the morphine is encapsulated in lipid foam particles (Figure 8) has been developed for epidural use. The encapsulation of the water-soluble morphine slows release resulting in a prolonged and sustained drug delivery and a dose-dependent half-life. The few clinical studies performed to date show analgesia extending into the second post-operative day, without significant side effects. When extended release epidural morphine 5, 10 or $15 \mathrm{mg}$ was compared to a standard $5 \mathrm{mg}$ dose of epidural morphine sulphate, the supplemental opioid dose used during the 24-48 hours post-operatively was significantly decreased in the 10 and $15 \mathrm{mg}$ groups $(25+/-21 \mathrm{mg}$ vs. 47 +/- $34 \mathrm{mg}$ in the $10 \mathrm{mg}$ extended release and $5 \mathrm{mg}$ standard group respectively) (Figure 9) (Carvalho, Riley et al. 2005). Area under the curve analysis across the first 48 hours showed less pain in the 10 and $15 \mathrm{mg}$ groups. A comparison of a $10 \mathrm{mg}$ extended release epidural morphine dose with a $4 \mathrm{mg}$ standard epidural morphine dose reported a significant reduction in 48 hour opioid use, although this appeared clinically unimportant $(10 \pm 17 \mathrm{mg}$ morphine equivalents vs. $17 \pm 22 \mathrm{mg}, \mathrm{p}=0.037$ ) (Carvalho, Roland et al. 2007). However, pain relief was better after 24 hours, without an increase in side effects. This prolonged effect into the period of full patient activity on the second post-operative day is attractive, but as the lipofoam may become unstable in the presence of local anaesthetic, its safety for women who have had epidural analgesia during labour is not yet known. The formulation is expensive and it will be difficult for many institutions to justify the additional cost when cheaper alternatives are available for analgesia on the second post-operative day. 
Figure 8: Depodur lipofoam particles

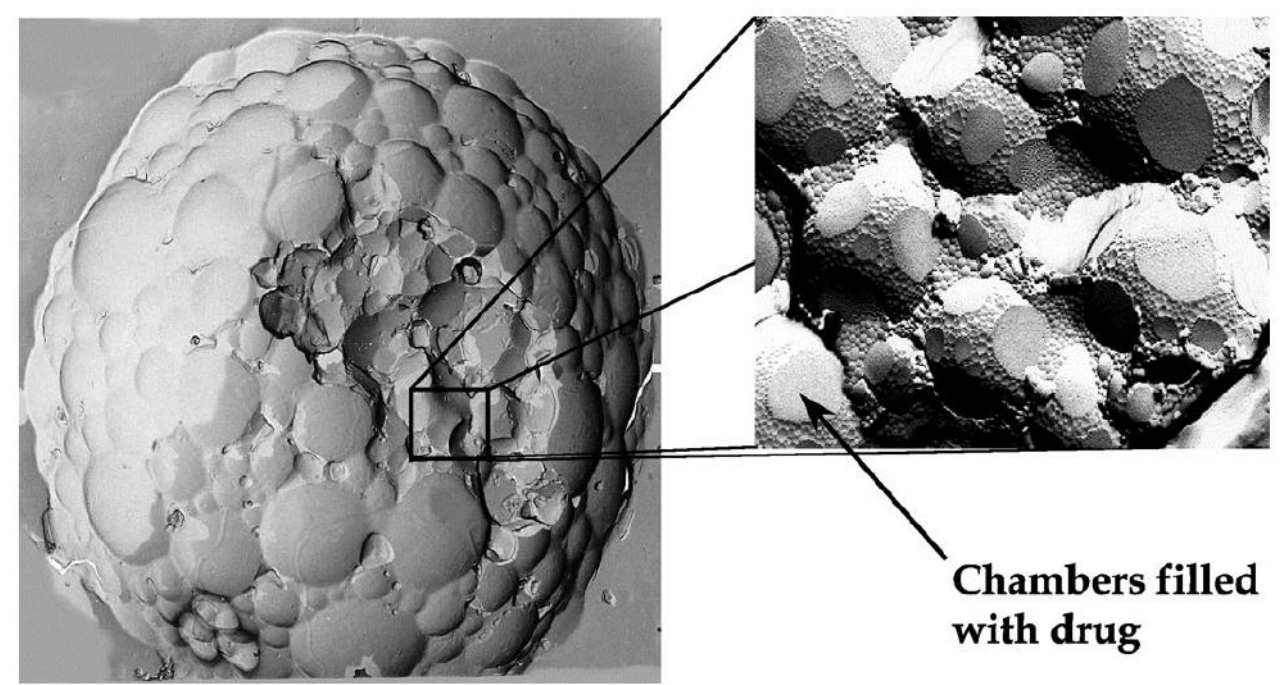

Electron micrograph of Depofoam particles. The nonconcentric vesicles are surrounded by a lipid membrane, and each contains an internal aqueous chamber with encapsulated morphine sulphate solution.

From: Carvalho et al. Anesth Anal 2005;100:1150-1158

Figure 9: Supplemental analgesia with extended release epidural morphine and conventional epidural morphine

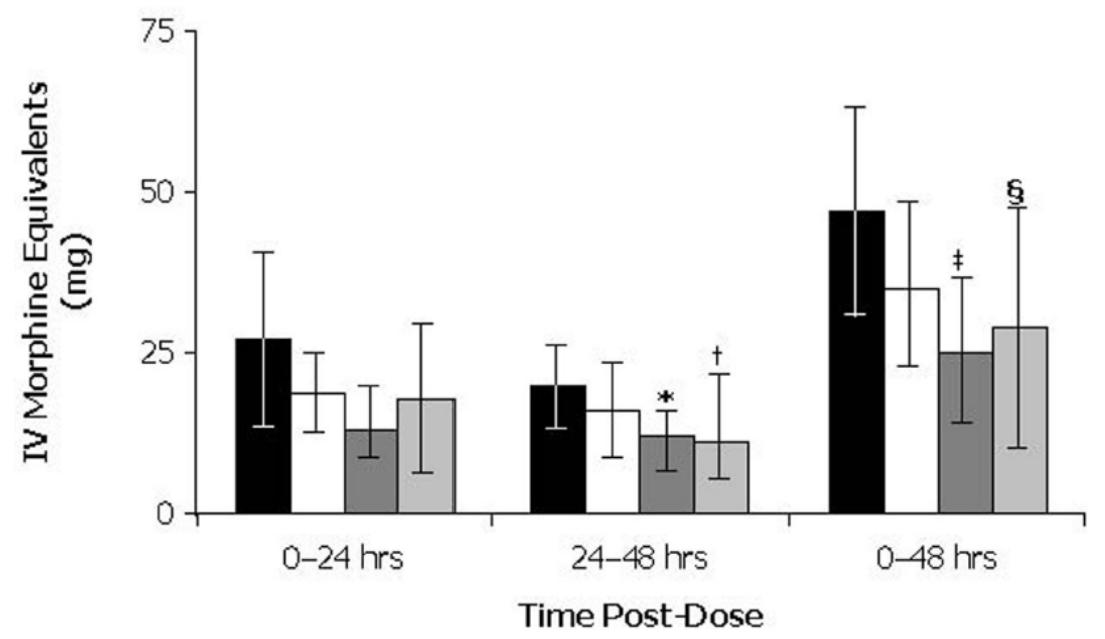

\begin{tabular}{|ll|}
\hline MOrphine $5 \mathrm{mg}$ & QEREM $5 \mathrm{mg}$ \\
aEREM $10 \mathrm{mg}$ & पEREM $15 \mathrm{mg}$ \\
\hline
\end{tabular}

Use of supplemental opioid analgesia (in morphine milligram equivalents) during the $48 \mathrm{~h}$ after the study dose. ${ }^{*} \mathrm{P}=0.0134$

From: Carvalho et al. Anesth Anal 2005;100:1150-1158 


\subsubsection{Neuraxial adjuncts}

\subsubsection{Clonidine}

A number of non opioid analgesics have been used in conjunction with epidural and intrathecal opioids to optimise post-operative analgesia. Of these drugs clonidine, an $\alpha_{2}$ adrenergic receptor agonist, has been the most widely studied. Its actions are enhanced by pregnancy (Iwasaki, Collins et al. 1991) and it appears to be particularly effective for visceral pain (Sabetkasaie, Vala et al. 2004). In obstetric patients intrathecal clonidine slows the regression of the sensory block after spinal anaesthesia, delaying the onset of post-operative pain (Figure 10) (Bonnet, Buisson et al. 1990; Eisenach, De Kock et al. 1996). Clonidine alone, in doses up to 150 mcg, does not provide sufficient post-operative pain relief, so must be combined with neuraxial morphine or other techniques (Paech, Pavy et al. 2004). With intrathecal morphine, clonidine 30 to $60 \mathrm{mcg}$ decreases overall opioid requirement and increases the duration of analgesia, but also increases intraoperative sedation (Paech, Pavy et al. 2004). Epidural clonidine in doses of between 75 and $150 \mathrm{mcg}$ has similar sedative effects (Capogna, Celleno et al. 1995). When combined with a modest dose of epidural morphine $(2 \mathrm{mg})$ analgesia was prolonged from $6.3 \mathrm{~h}$ (control) to $13.3 \mathrm{~h}(75 \mathrm{mcg})$ and $21.5 \mathrm{~h} \mathrm{(150} \mathrm{mcg)} \mathrm{(Capogna,} \mathrm{Celleno} \mathrm{et} \mathrm{al.} \mathrm{1995).}$

\subsubsection{Neostigmine}

Neuraxial neostigmine, by inhibiting acetylcholinesterase and preventing the breakdown of acetylcholine in the spinal cord interneurons, provides effective analgesia with no motor or sympathetic blockade (Kaya, Sahin et al. 2004). When administered intrathecally neostigmine causes severe nausea and vomiting (74\% incidence with a $25 \mathrm{mcg}$ dose) which precludes its use by this route (Chung, Kim et al. 1998). In contrast, epidural neostigmine is not associated with an increased risk of post-operative nausea and vomiting and 75-300 mcg doses result in modest analgesia in women post-caesarean (global pain scores 3.0-3.5 \pm 0.3 in the neostigmine groups vs. $5.4 \pm 0.2$ in the placebo group) (Kaya, Sahin et al. 2004). Higher doses of neostigmine increase sedation but do not appear to increase nausea and vomiting. More studies in obstetric patients are required to validate these results and to ascertain whether there is a benefit from adding neostigmine to epidural solutions for post-caesarean analgesia. 
Figure 10: Duration of analgesia with intrathecal morphine and clonidine combinations

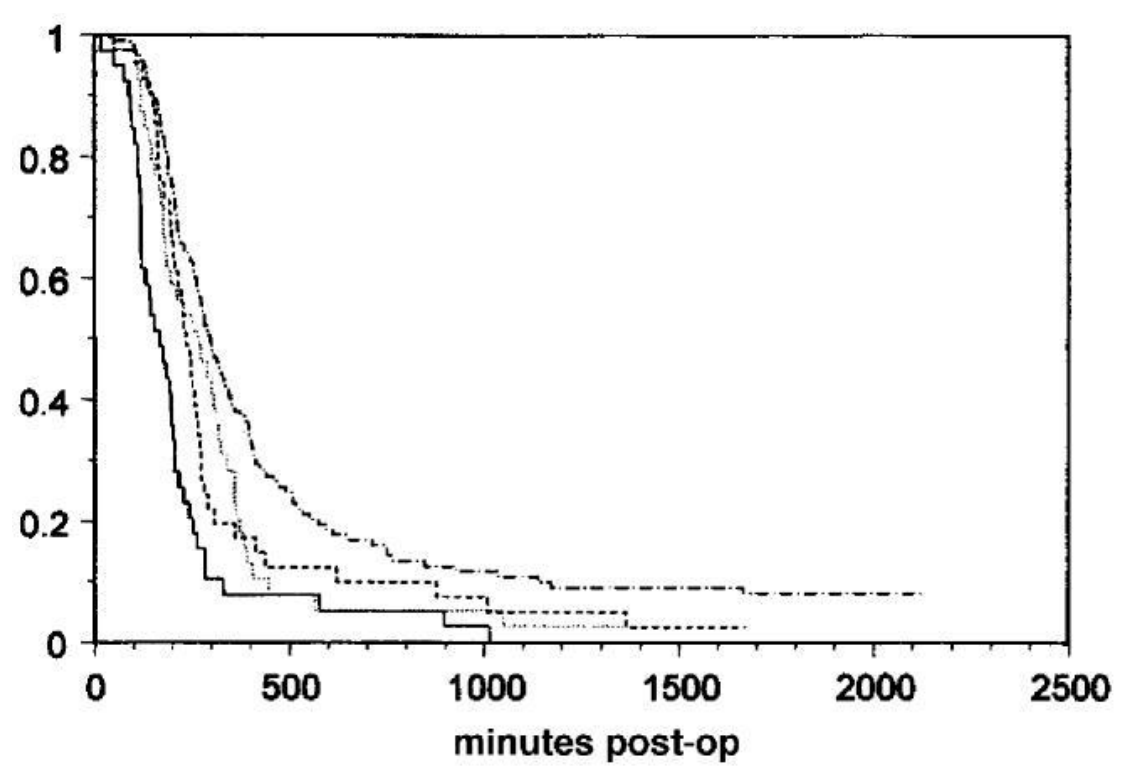

The duration of analgesia as shown by the time to first activation of the PCA device showing prolonged duration with morphine $100 \mathrm{mcg}$ and clonidine $60-150 \mathrm{mcg}$ (dot-dashed line) compared to morphine $100 \mathrm{mcg}$ and clonidine $30 \mathrm{mcg}$ (dotted line), morphine $100 \mathrm{mcg}$ (dashed line) and clonidine $150 \mathrm{mcg}$ (unbroken line). From: Paech et al. Anesth Analg 2004;98:1460-1466 


\subsection{Intravenous, intramuscular and subcutaneous opioids}

Patient controlled intravenous opioids are popular after caesarean delivery because of convenience, safety and consistently high patient satisfaction. In comparison with neuraxial opioids it appears that intravenous opioids are inferior with respect to the quality of pain relief but they are often rated favourably in terms of patient satisfaction, most likely due to the increased autonomy that comes from administration by a patient controlled technique (Eisenach, Grice et al. 1988). This is demonstrated when patient controlled neuraxial and intravenous opioid delivery are directly compared; in this situation neuraxial opioids are more likely to be preferred (Paech, Moore et al. 1994). Morphine is popular for intravenous PCA in obstetrics and is often used as a standard against which other interventions are evaluated (Lavand'homme 2006). Fentanyl is also an appropriate systemic opioid, in contrast to pethidine, which is rarely used today secondary to accumulation of its active metabolite in breast milk and adverse effects on the newborn. The combination of alfentanil and morphine may also hold advantages, especially in relation to speed of onset (Ngan Kee, Khaw et al. 1999).

Patient controlled intravenous or neuraxial techniques have a number of advantages. These include catering for the wide interpatient variation in analgesic requirements and reducing staff workload. More stable plasma drug concentrations are likely to result in more reliable pain relief than that associated with nurse administered techniques, although higher concentrations may increase the incidence of opioid related side effects. Patient mobilisation may be restricted unless a lightweight, portable device is used (Mcdonnell, Kwei et al. 2007). The potential for device malfunctions and programming errors can also lead to adverse patient outcomes (Vicente, Kada-Bekhaled et al. 2003).

A previous review has suggested that intramuscular and subcutaneous opioids are the most commonly used methods of post-caesarean pain relief although evidence for this is lacking (Gadsden, Hart et al. 2005). Whilst being cheap and simple to administer they have a number of disadvantages. Blood levels of opioid are likely to vary considerably between individuals, the injection may be painful, the method labour intensive and women are likely to be reluctant to request further doses. 
These methods are considered outdated in clinical practice, are rarely used in clinical studies and poorly reported in the literature.

\subsection{Non steroidal Anti-inflammatory Drugs}

Non steroidal anti-inflammatory analgesics (NSAIDs) are particularly effective against the visceral pain that arises from the uterine incision and uterine involution following caesarean delivery. They have a well documented opioid sparing effect, with a consequent reduction in opioid related side effects (Pavy, Gambling et al. 1995; Cardoso, Carvalho et al. 1998). Dahl et al investigated rectal diclofenac, 100 $\mathrm{mg}$ bd, and found a reduction in the post-operative morphine consumption from $21.5 \mathrm{mg}$ to $14.0 \mathrm{mg}$ in the first 32 hours (Dahl, Hagen et al. 2002). Cardoso et al combined intramuscular diclofenac with intrathecal morphine and supplemental analgesia was avoided, even at intrathecal morphine doses of $25 \mathrm{mcg}$ (Cardoso, Carvalho et al. 1998). Pavy et al demonstrated a 30\% opioid- sparing effect from intravenous ketorolac $120 \mathrm{mg}$ over 24 hours, but ketorolac now has an FDA 'black box' warning for obstetric use because of concerns about the effect of fetal and neonatal exposure (Pavy, Paech et al. 2001). When Pavy et al compared rectal indomethacin (100 mg bd) with placebo in conjunction with intrathecal morphine, median time to first analgesia was extended from 9 hours in the control group to 39.5 hours in the indomethacin group $(\mathrm{P}<0.003)$ with enhanced pain relief during movement (mean VAS scores 1.4 vs. 5.1, P<0.001) (Pavy, Gambling et al. 1995).

Traditional NSAIDs have effects on platelet, renal and gastro-intestinal function and are relatively contra-indicated in the post partum period in women suffering from pre-eclampsia, because of their potential to worsen hypertension, precipitate a hyptertensive crisis or compromise renal blood flow. Concern has also been raised in regards to uterine atony in the post partum period, with case reports of severe uterine atony associated with ketorolac (Anderson 2006). Although there is insufficient evidence for a causal relationship, the use of NSAIDs in women at risk of postpartum haemorrhage deserves consideration.

Substitution of an NSAID with a cyclo-oxygenase 2 (COX 2) specific inhibitor has the potential to decrease side effects, particularly in regards to the platelet and gastrointestinal systems, but research in obstetrics is very limited. Carvalho et al studied valdecoxib post-caesarean but the study was stopped early as a result of 
emerging safety concerns about the COX 2 agents (Carvalho, Chu et al. 2006). No difference from placebo was seen in the time to first analgesic use or total opioid use (143 mg morphine equivalents in the placebo group vs. $121 \mathrm{mg}$ in the valdecoxib group). Despite the study being stopped early, their post hoc power analysis suggested that they should have had enough subjects to show a difference in pain scores and analgesic consumption, should one have existed. The COX 2 inhibitors such as celecoxib appear to be safe to use during breast feeding and a study is currently underway in the authors own institution to examine the safety of parecoxib during the initiation of breast feeding.

\subsection{Paracetamol}

Paracetamol may also work through central COX 2 inhibition, by reducing central nervous system (CNS) prostaglandin E2 production and by activating descending serotonergic pathways (Lavand'homme 2006). Studies in the obstetric population are few and have reported conflicting results. Alhashemi et al compared $1 \mathrm{~g}$ intravenous paracetamol with $400 \mathrm{mg}$ oral ibuprofen post-caesarean and found no difference in 48 hour morphine consumption $(98 \pm 37$ and $93 \pm 33 \mathrm{mg}$ for paracetamol and ibuprofen respectively) (Alhashemi, Alotaibi et al. 2006). Siddik et al found a significant morphine sparing effect with rectal diclofenac but not IV proparacetamol and no additional benefit when both were combined (Siddik, Aouad et al. 2001). In contrast, Munishankar et al demonstrated that the combination of paracetamol and diclofenac resulted in significantly less morphine consumption than paracetamol alone (33.8 mg vs. $54.5 \mathrm{mg}$ ) (Munishankar, Fettes et al. 2008). Despite these conflicting results, there is little risk associated with paracetamol therapy and it has been suggested as "near-routine" for post-operative pain management (Hyllested, Jones et al. 2002). The release of IV paracetamol may offer advantages, particularly for early post-operative analgesia and patient acceptance in comparison with rectal administration.

\subsection{Wound infiltration and nerve blocks}

A significant component of the pain experienced after caesarean delivery arises from the surgical incision through the anterior abdominal wall. This can be blocked with a number of local anaesthetic techniques, including ilioinguinal and iliohypogastric nerve blocks, wound instillation and the recently described 
transversus abdominis plane (TAP) block. The potential advantages of these techniques are that they are less invasive than neuraxial blocks (and hence potentially associated with a lower complication rate), are suitable for patients having general anaesthesia and can also be repeated post-operatively if required. A recently published Cochrane review (July 2009) that included 20 studies with 1150 women confirmed the analgesic efficacy of local anaesthetic based techniques, but the magnitude of effect (and hence the clinical efficacy) was dependant on a number of factors (Bamigboye and Hofmeyr). Women having wound infiltration at the conclusion of a caesarean performed under regional anaesthesia had only a clinically unimportant decrease in 24 hour morphine consumption of $1.7 \mathrm{mg}$. In contrast, women who had abdominal wall nerve blocks at the conclusion of regional anaesthesia had a mean decrease in 24 hour morphine consumption of $25.8 \mathrm{mg}$ (95\% CI -50.39- -5.37). In women undergoing general anaesthesia who had wound infiltration combined with peritoneal spraying, the need for opioid rescue was decreased (RR 0.51, 95\% CI 0.38-0.69), with a decrease in the 1 hour numerical pain score (0-10 scale) of 1.46 (95\% CI -2.6- -0.32).

Despite evidence of efficacy, ilioinguinal/iliohypogastric nerve blocks do not appear to be widely used, possibly because of high rates of block failure (up to $50 \%$ in some studies) (Huffnagle, Norris et al. 1996; Bell, Jones et al. 2002) and a relatively short duration of action. A continuous technique, using ultrasound guidance and continued via a catheter for 72 hours post-operatively has recently been described with excellent results (Gucev, Yasui et al. 2008) but was a very small case series of only three patients, so the encouraging results require further investigation.

Wound infusion catheters are used in a number of post-operative settings but results after abdominal surgery have been mixed. This is probably due to differences in the site of catheter placement, the drugs used and the outcome from continuous versus bolus techniques. Ranta et al, in a double blind study, compared epidural analgesia and subfascial wound catheters using an intermittent bolus technique (Ranta, Ala-Kokko et al. 2006). Pain scores in the epidural group were lower at 4 hours post-operatively after which both groups had pain scores of 3 or less. Fredman et al investigated a patient controlled elastomeric wound infusion device (10 $\mathrm{ml} \mathrm{0.2 \%} \mathrm{ropivacaine} \mathrm{hourly} \mathrm{on} \mathrm{request)} \mathrm{in} \mathrm{a} \mathrm{placebo} \mathrm{controlled} \mathrm{trial} \mathrm{and} \mathrm{found}$ decreased pain with movement and lower opioid requirements in the intervention group (Fredman, Shapiro et al. 2000). In contrast, Zohar et al found no benefit 
from regular intermittent bolus dosing of bupivacaine into the surgical wound via an epidural catheter placed at the conclusion of surgery (Zohar, Shapiro et al. 2006).

Interestingly, diclofenac (300 mg over 48 hours) via a wound infusion catheter decreases 48 hour morphine requirements compared with ropivacaine infusion or IV diclofenac (18 mg (95\% CI 12.7-22.2) in the diclofenac wound infusion group compared with $28 \mathrm{mg}$ (95\% CI 18.2-32) in the ropivacaine/IV diclofenac group and $38 \mathrm{mg}$ (95\% CI 28.8-43.7) in the saline/IV diclofenac group) (Lavand'homme, Roelants et al. 2007). This raises the possibility that diclofenac has peripheral analgesic properties. Whilst wound infusion catheters provide prolonged drug delivery, the optimal site of placement is unknown. The relatively high cost of some disposable devices and the effort required to prepare and insert these catheters has likely limited their widespread acceptance.

The transversus abdominis plane (TAP) block is a relatively recently described technique for blocking the neural afferents supply to the anterior abdominal wall (McDonnell, O'Donnell et al. 2007). With this block local anaesthetic is injected into the neurofascial plane between the internal oblique and the transversus abdominis muscles (Figure 11). The surface landmarks are relatively consistent between individuals (McDonnell, O'Donnell et al. 2007), and the passage through the tough fascia of the abdominal wall muscles provides a definitive endpoint, which can also be visualised using ultrasound. McDonnell et al evaluated 50 women with a Pfannenstiel incision and randomised them to a bilateral TAP block (landmark technique) using ropivacaine $0.75 \%(1.5 \mathrm{mg} / \mathrm{kg}$ to a maximum of $150 \mathrm{mg}$ per side) or a saline placebo (McDonnell, Curley et al. 2008). The median time to first morphine request was extended from 90 to 220 minutes in the TAP block group (Figure 12). The TAP block group also had lower morphine requirements in the first 48 hours (18 mg vs. $66 \mathrm{mg}, \mathrm{p}<0.001)$ (Figure 13) and a corresponding reduction in sedation and nausea. These initial results are encouraging but because the blocks were performed by a single experienced operator, further research is required to ascertain whether these findings are reproducible in other centres, as well as whether there is any benefit to performing the block under ultrasound guidance. 
Figure 11: Anatomical diagram of the abdominal layers and the TAP Block

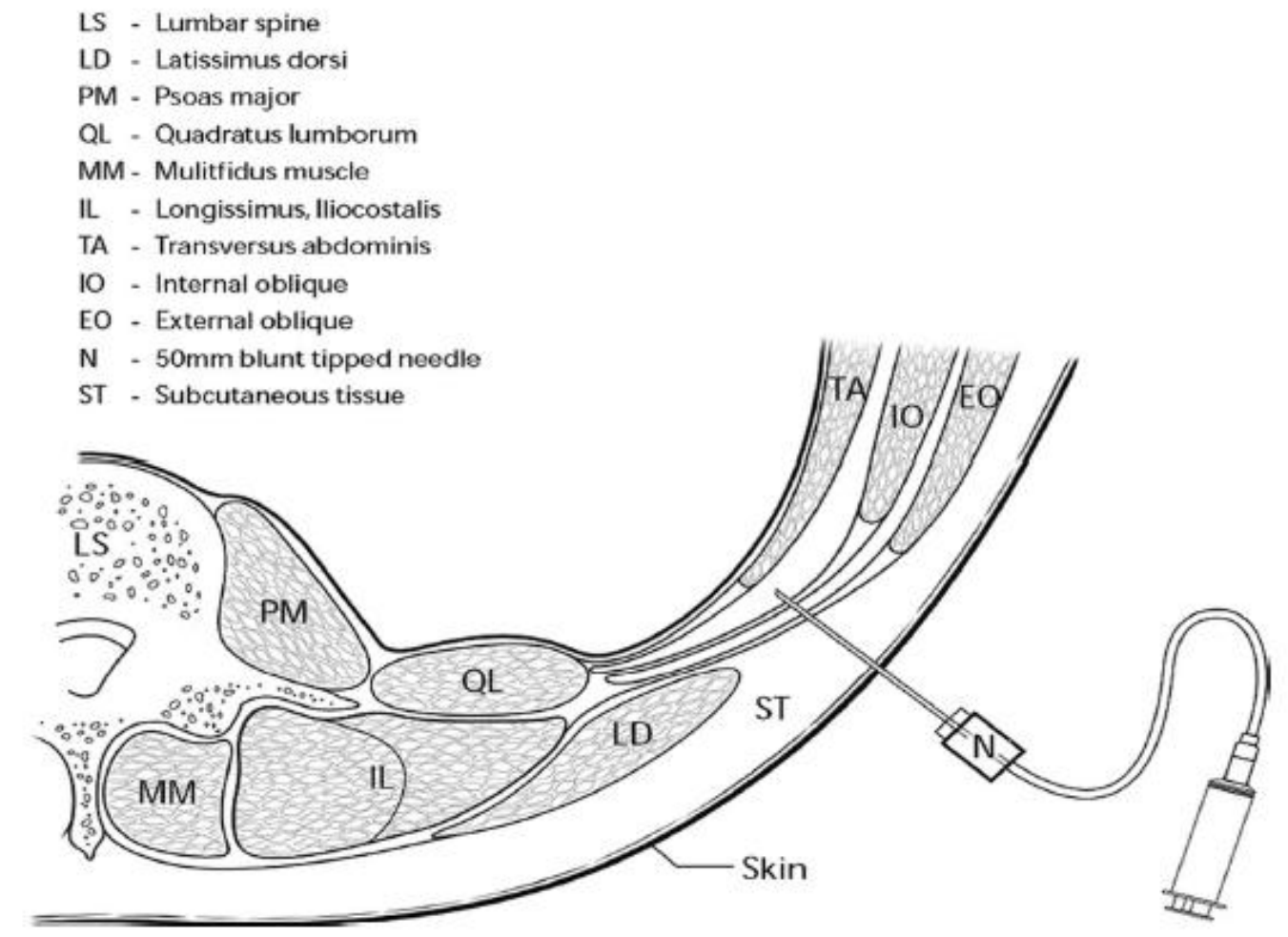

Line drawing of a transverse section through the abdominal wall at the level of the lumbar triangle of Petit. The floor of the triangle is composed, from superficial to deep, of the fascial extensions of external oblique, internal oblique, and transversus abdominis, respectively, and the peritoneum. The needle is inserted through the triangle, using the loss-of-resistance technique. The needle is shown in the transversus abdominis plane, and the fascial layers have separated as a result of the injection of local anesthetic.

From: McDonnell et al: Anesth Analg 2008;106:186-191 
Figure 12: Time to first analgesic request in TAP block versus control subjects

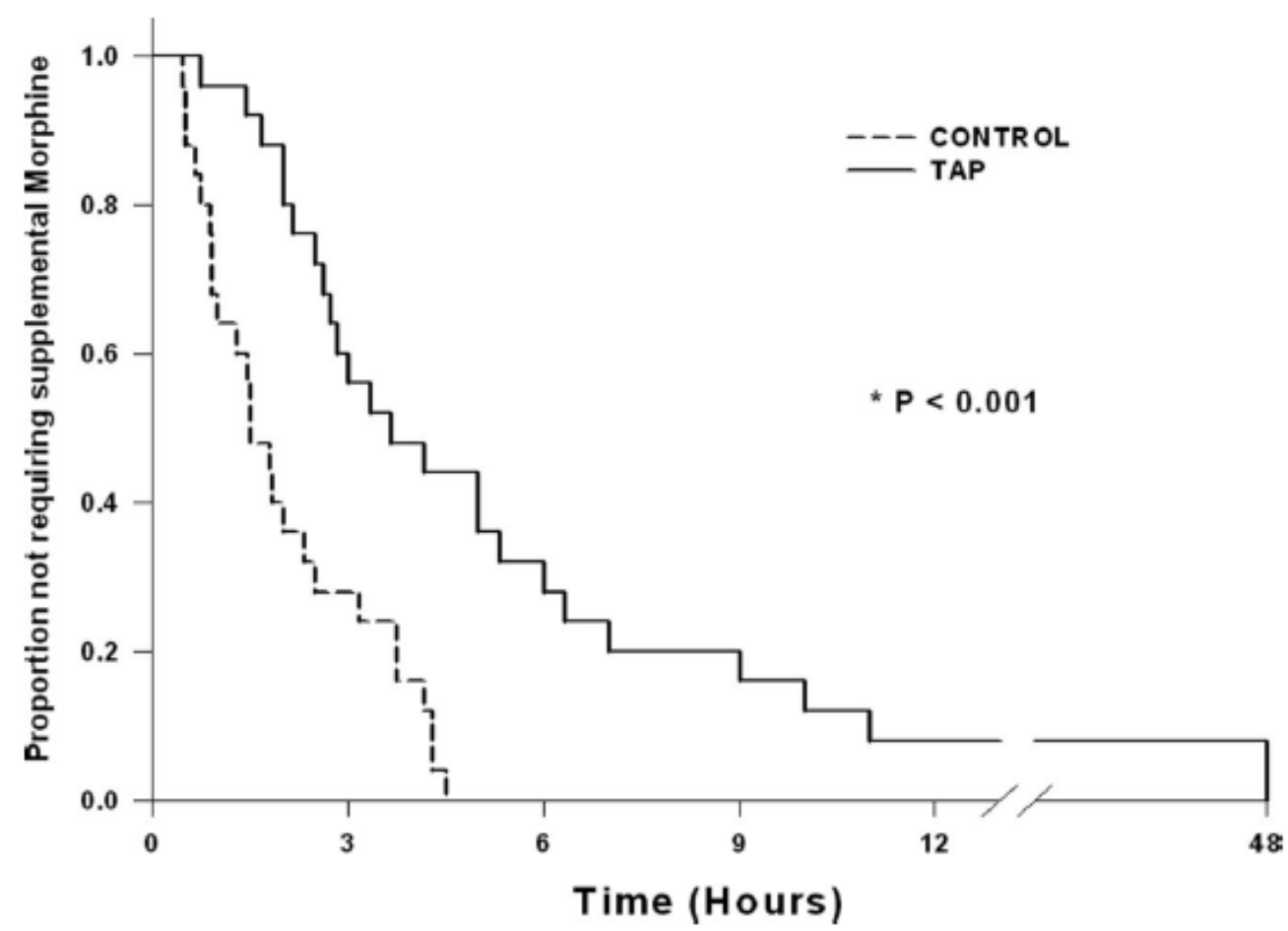

A Kaplan-Meier graph of the proportion of patients in each group over time who did not require supplemental morphine (P 0.001, log rank test).

From: McDonnell et al: Anesth Analg 2008;106:186-191 
Figure 13: Morphine consumption post-caesarean with the TAP block versus control subjects

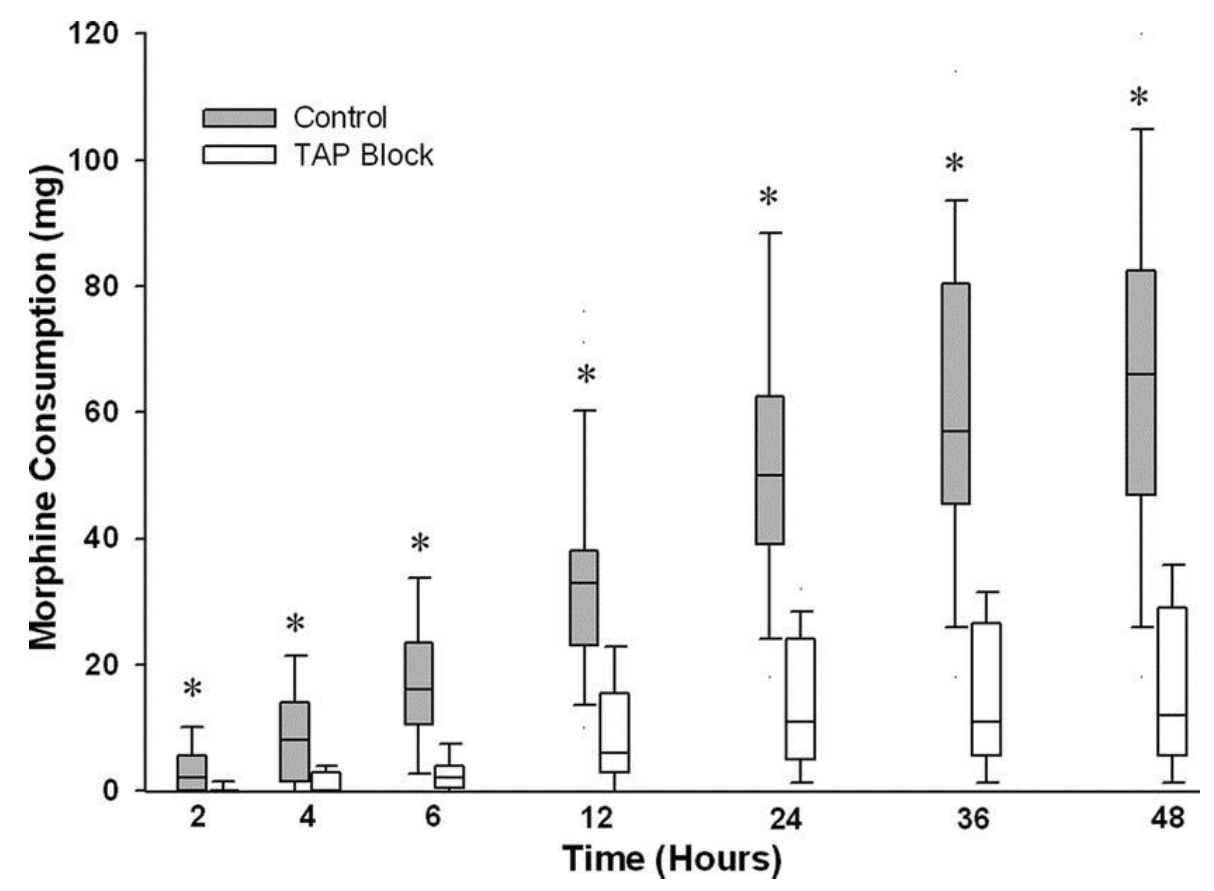

Post-operative cumulative morphine consumption (median, interquartile range, $10^{\text {th }}$ and $90^{\text {th }}$ percentiles) during the first 48 hours post-caesarean section in women receiving the transversus abdominis block. ${ }^{*} \mathrm{p}<0.05$.

From: McDonnell et al: Anesth Analg 2008;106:186-191

\subsection{Chronic post-caesarean pain}

Whilst acute post-caesarean pain has been widely studied, persisting pain after caesarean delivery has not. The incidence of chronic pain is now known to be high after some surgical procedures, with even relatively minor surgery such as gall bladder surgery and inguinal hernia repair leading to reported rates of chronic pain of between 10-20\% (Callesen, Bech et al. 1999; Perkins and Kehlet 2000; BayNielsen, Perkins et al. 2001; Nikolajsen, Sorensen et al. 2004; Bisgaard, Rosenberg et al. 2005). To date only one study has examined chronic post-caesarean pain. Nikolajsen et al studied 220 patients by written, posted questionnaire (Nikolajsen, Sorensen et al. 2004). At the 3 month review, pain had resolved in the majority of women but surprisingly, $18.6 \%$ of women were still experiencing abdominal scar pain. The mean follow-up time was 10.2 months, at which point pain was still present in $12.3 \%$ of patients, $5.9 \%$ experiencing daily pain. Patients were more likely to have persistent pain if they had their caesarean performed under general 
anaesthesia, had greater recall of severe post-operative pain or had pain problems elsewhere. The authors question the role of central sensitization in the development of chronic post-caesarean pain, as well as genetic and psychosocial susceptibility to pain.

\subsection{Conclusions}

A number of options are available for post-caesarean analgesia. Currently, opioids form the foundation of most techniques, with patient controlled methods being preferred by mothers. There has been considerable work performed on intrathecal morphine, and to a lesser extent on epidural morphine, while patient controlled intravenous morphine is commonly used as a comparator in many studies. The choice of opioid is likely to be subject to a number of local and resource considerations. Oral opioids have not been the subject of an appropriately powered and designed clinical study, although audit data and data from relatively low quality studies would suggest that it is an acceptable route of administration with a potentially favourable side effect profile. In addition to opioids, neuraxial clonidine, and to some extent epidural neostigmine, enhance post-operative analgesia with few untoward side effects. NSAIDs and paracetamol add additional benefit but are not appropriate in a number of obstetric situations. Nerve blocks have demonstrated analgesic benefits post-caesarean and of the currently available techniques, the TAP block appears the most promising but requires further validation. Finally, while acute post-caesarean pain has been extensively studied, little work has focused on chronic post-caesarean pain and this should be the subject of future studies. 


\section{Chapter Two: Primary Oral Analgesia Post- \\ Caesarean Delivery}

\subsection{Introduction}

Opioids, administered by the intravenous or neuraxial route, form the foundation of most multimodal analgesic regimens post-caesarean delivery (Pan 2006). The side effects of opioid use depend on the route of administration as well as the specific opioid in use (Chaney 1995). Oral non-opioid medications are frequently used to supplement opioid analgesia in the post-caesarean period (Lavand'homme 2006; Pan 2006). Paracetamol in combination with NSAIDs form the foundation of these adjunctive techniques. Oral opioids are commonly used as step down or supplemental agents post-caesarean. In this situation they are associated with good maternal acceptability, likely due to familiarity with this route of administration. However they are not commonly used as the primary analgesic approach on the first or second day post-caesarean. This may be because of a perceived lack of efficacy compared with alternative routes of administration as well as potential concerns in regards to respiratory depression when combined with other neuraxial opioids (Kato, Shimamoto et al. 2008). Recently, evidence has emerged that using oral opioids as the primary route of administration for post-caesarean analgesia may have benefits such as ease of administration, superior pain relief, a better side effect profile and improved maternal satisfaction compared with intravenous administration. The evidence come from from audit data in centres that use Oramorph as well as three clinical studies (Holt 2000; Jakobi, Weiner et al. 2000; Antrobus 2001; Scott, Cameron et al. 2001; Jakobi, Solt et al. 2002; Davis, Esposito et al. 2006). These data only provide limited information. Whilst the audit data appears promising, there have not been comparison groups with which to compare regimens. The clinical studies that have been performed can be criticized for the lack of blinding, inadequate assessment of post-operative pain and poor choices of regimens among comparison groups.

Given the possible benefits of an oral opioid based regimen, a well designed clinical trial that compared oral opioid administration to a commonly used and highly validated intrathecal opioid regimen was planned. 


\subsubsection{Hypothesis}

2.1.1.1 Null Hypothesis: That there is no difference in the post-caesarean pain scores between a multimodal regimen based on intrathecal morphine and a multimodal regimen based on oral oxycodone.

2.1.1.2 Alternative Hypothesis: The alternative hypothesis is that a multimodal regimen based on intrathecal morphine shows superiority by resulting in significantly lower pain scores than one based on oral oxycodone.

\subsubsection{Aims}

1. To compare the quality of post-operative pain relief provided by a standard multimodal analgesic regimen based on intrathecal morphine with that provided by one based on regular oral administration of oxycodone, for women receiving regional anaesthesia for caesarean delivery.

2. To evaluate the side effects of a regular fixed dose oral opioid based regimen.

3. To evaluate the satisfaction with such an approach in women postcaesarean.

\subsection{Methods}

The study was performed at King Edward Memorial Hospital for Women in Perth, Western Australia. Prior to the study commencing, Institutional Ethics Committee approval was received from the Women's and Children's Health Service, Department of Health, Government of Western Australia (Approval date $5^{\text {th }}$ September 2006, registration number 1297/EW, reference number EC06-57) and written informed consent was obtained from all participants prior to their involvement. The trial was registered with the Australian New Zealand Clinical Trials Registry (Registration number ACTRN12606000506594, www.anzctr.org.au). Women were recruited from the antenatal clinics, the pre-admission clinics and the antenatal and assessment wards. A letter introducing the study and containing the 
patient information sheet was sent to eligible women at least one week prior to their pre-admission appointment.

\subsubsection{Study design}

The study was designed as a randomised, double-blind, double-dummy, placebo controlled trial of intrathecal morphine versus oral oxycodone. The study was designed as a superiority trial of intrathecal morphine over oral oxycodone.

\subsubsection{Study Endpoints}

\subsubsection{Primary endpoint:}

1. The efficacy of the analgesic regimen, as determined by the area under the curve for pain scores over 0-24 hours.

\subsubsection{Secondary endpoints:}

1. The pain score with movement (sitting erect from the supine position) at 6,12 , 18 and 24 hours post-operatively.

2. The pain score at rest at $6,12,18$ and 24 hours post-operatively.

3. The incidence of nausea and vomiting

4. The severity of nausea

5. The incidence and severity of pruritus

6 . The severity of maternal sedation

7. The maternal satisfaction with the analgesic regimen

8. The incidence of additional supplementation with oral oxycodone and oral tramadol

9. The Quality of Recovery Score (Myles, Hunt et al. 1999)

10. The Modified Brief Pain Inventory (Mendoza, Chen et al. 2004)

11. The opioid-Symptom Distress Scale (Apfelbaum, Gan et al. 2004)

\subsubsection{Participants}

\subsubsection{Inclusion criteria}

1. American Society of Anesthesiologists (ASA) 1 or 2

2. Planned elective caesarean delivery under combined spinal epidural (CSE) anaesthesia

3. Aged 18 years and over 


\subsubsection{Exclusion criteria}

1. Pre-operative use of opioid medication

2. Contraindication to combined spinal epidural anaesthesia

3. Contraindication to diclofenac or tramadol

4. Known intolerance or allergy to oxycodone, morphine or diclofenac

5. Pre-operative pruritus or nausea

6. Failure to identify the subarachnoid space at the time of combined spinal epidural anaesthesia

7. Inadvertent dural puncture with the epidural needle

8. Conversion of spinal anaesthesia to general anaesthesia

Women undergoing caesarean delivery under CSE anaesthesia rather than single shot spinal anaesthesia were recruited for logistical reasons. In this hospital women are anaesthetised for their caesarean in a room adjacent to the operating theatre. Not infrequently there are delays in the commencement of surgery as a consequence of either delays in the operating theatre or more urgent cases taking priority. Also, because of the teaching and training roles of the institution, the duration of surgery can be prolonged. The use of a CSE technique allows for extension of the regional block through the use of the epidural catheter as the spinal anaesthesia regresses. Otherwise, with a single shot spinal anaesthetic technique, a delay to the surgical start time may require a second procedure to be performed, and if there is intraoperative pain due to block recession near the end of surgery, then there is a greater likelihood of general anaesthesia being required. In the study, no opioid or other non opioid analgesic drug was to be administered via the epidural catheter, the catheter being removed at the conclusion of surgery.

\subsubsection{Group assignment and study drugs}

Pre-operatively, subjects were randomised into one of two groups (Group $\mathrm{O}$ and Group I) using a random number generator prepared by the hospital pharmacy department. The hospital pharmacy prepared individual patient packs containing the study and placebo medications of identical appearance. By this means, all patients, staff and investigators were blinded to the group allocations. The intrathecal study drug was also prepared by the hospital pharmacy, after a three month quality assurance process to ensure sterility of the samples, and these intrathecal solutions 
were presented in $1 \mathrm{ml}$ syringes containing $1.0 \mathrm{ml}$ of the study solution, either normal saline (Group O) or $500 \mathrm{mcg}$ preservative free morphine sulphate (Group I). Of this solution, $0.2 \mathrm{ml}$ was to be administered intrathecally at the time of the establishment of spinal anaesthesia.

Patients in Group O received post-operative analgesia based on regular, fixed dosing with oral oxycodone. They received a $0.2 \mathrm{~mL}$ placebo intrathecal injection of sterile saline at the time of spinal anaesthesia and $20 \mathrm{mg}$ of sustained-release oral oxycodone immediately on arrival in the recovery room. This was followed by immediate-release oral oxycodone $10 \mathrm{mg}$ every $6 \mathrm{~h}$ post-operatively, in conjunction with oral paracetamol $1 \mathrm{~g}$.

Patients in Group I received preservative-free intrathecal morphine $100 \mathrm{mcg}(0.2$ $\mathrm{mL})$ at the time of spinal anaesthesia and matching oral placebo medication, instead of oxycodone, in conjunction with oral paracetamol $1 \mathrm{~g}$ at the same time intervals as group O (Table 1).

\subsubsection{Pre-operative preparation}

All patients received aspiration prophylaxis with oral ranitidine $300 \mathrm{mg}$ on the morning of surgery, were fasted from solid food from midnight and allowed clear oral fluids up until $3 \mathrm{~h}$ before surgery.

\subsubsection{Management of anaesthesia and analgesia}

In both groups CSE anaesthesia was achieved through a low lumbar interspace in the seated position using a 16-gauge Tuohy needle and 27 gauge pencil point spinal needle. Spinal anaesthesia was established with hyperbaric bupivacaine 11-12.5 mg (with the dose within this range being at the discretion of the attending anaesthetist), $15 \mathrm{mcg}$ fentanyl and $0.2 \mathrm{ml}$ of the intrathecal study solution. To ensure sterility and consistency of the volume of intrathecal study drug administered to the patient, a standardised approach was used. The intrathecal study solution was presented in a $1 \mathrm{ml}$ non sterile syringe from the pharmacy. This was transferred to a $1 \mathrm{ml}$ sterile syringe by the use of a sterile connector. A 22 gauge needle was then attached to the sterile $1 \mathrm{ml}$ syringe by the procedural anaesthetist and the needle was primed with study solution. A reference point on the syringe was then noted and 0.2 $\mathrm{ml}$ was added to the $3 \mathrm{ml}$ syringe containing the rest of the intrathecal medications. 
The combined spinal epidural procedure then continued in the usual fashion. After securing of the epidural catheter the patient was placed flat on the bed with a wedge underneath the right hip. Haemodynamic stability was maintained with a continuous infusion of phenylephrine, commencing at $50 \mathrm{mcg}$ per min, to maintain a blood pressure within 10-20\% of baseline. Cephazolin $2 \mathrm{~g}$ IV was administered predelivery for antibiotic prophylaxis against wound infection.

After establishment of adequate spinal anaesthesia, defined as a block to ice at the fourth thoracic (T4) dermatome, surgery was commenced and the neonate was delivered through a Pfannestiel incision. Intravenous dexamethasone $4 \mathrm{mg}$ was given as antiemetic prophylaxis immediately after delivery. All women received IV parecoxib $40 \mathrm{mg}$ at the conclusion of surgery. The epidural catheter was used only if surgery was delayed or if intraoperative pain experienced, in which case epidural lignocaine with adrenaline 1: 200000 was given, and in all patients it was removed at the conclusion of surgery. Other treatments for intraoperative pain that were allowed according to the protocol were inhaled nitrous oxide, intraperitoneal or intra-wound local anaesthetic infiltration or small doses of IV fentanyl or ketamine as appropriate.

\subsubsection{Post-operative management}

Patients were allowed free oral fluids after discharge from the recovery area and thereafter were permitted to eat solid food at the discretion of the attending midwife. Post-operatively, 8-hourly oral diclofenac $50 \mathrm{mg}$ was commenced at 12 hours after surgery. Supplemental post-operative analgesia was available as immediate release oral oxycodone, 10-15 mg 2-hourly on demand. If this proved insufficient (0-10 dynamic pain numerical rating score [NRS] > 6) then oral tramadol $100 \mathrm{mg}$ was also available. Post-operative pruritus was treated with IV ondansetron $4 \mathrm{mg}$ 6-hourly on demand or if this was ineffective with hourly IV naloxone $50 \mu \mathrm{g}$. Post-operative nausea and/or vomiting were treated with IV ondansetron or metoclopramide. 
Table 1 Intra and post-operative analgesia

Treatment regimen

Subarachnoid medication Hyperbaric bupivacaine 11-12.5 mg

Fentanyl $15 \mu \mathrm{g}$

Group I: morphine $100 \mu \mathrm{g}$; Group O: saline placebo

Intra-operative

Parecoxib $40 \mathrm{mg}$ i.v.

Recovery room

Paracetamol 1 g orally

Group I: oral placebo; Group O oxycodone SR 20 mg orally

Post-operative

Paracetamol 1 g orally

$6,12,18$ and $24 \mathrm{~h}$ post

Group I: placebo orally; Group O: oxycodone IR $10 \mathrm{mg}$ orally

recovery room medication

Diclofenac $50 \mathrm{mg}$ orally

8-hourly starting at least $12 \mathrm{~h}$

post-operatively

Rescue analgesia

Oxycodone IR 10-15 mg orally 2-hourly as required

(Dynamic NRS $>6$ )

Tramadol $100 \mathrm{mg}$ orally 4-hourly as required

$\mathrm{SR}=$ sustained release; $\mathrm{IR}=$ immediate release

Group $\mathrm{I}=$ intrathecal morphine; Group $\mathrm{O}=$ oral oxycodone 


\subsubsection{Assessments}

\section{$\underline{\text { Baseline }}$}

Baseline data was collected within 24 hours of the scheduled time of surgery, usually on the day of surgery. The data collected included:

1. Patient age

2. Weight

3. Gravida and parity

4. Gestation

5. The number of previous caesarean sections

6. Pre-operative quality of recovery (QoR) score were recorded.

Intra-operatively the data collected included:

1. The duration of surgery

2. The intrathecal drugs administered

3. The need for additional intraoperative analgesia.

The post-operative data collected included:

1. The time the oral medication was first given in recovery

2. NRS pain scores at rest and movement at $\mathrm{T}=6,12,18$ and 24 hours

3. NRS sedation scores at $\mathrm{T}=6,12,18$ and 24 hours

4. AUC for pain scores over 0-24 hours

At the 24 hour assessment by a blinded observer:

1. Presence of gastrointestinal upset, nausea or epigrastric pain

2. Maternal satisfaction with the analgesia provided (NRS 0-10)

3. Additional analgesia required (oral oxycodone and oral tramadol)

4. Global $24 \mathrm{~h}$ NRS nausea score

5. Global 24 h NRS sedation score

6. Global $24 \mathrm{~h}$ NRS pruritus score

7. Post-operative QoR score

8. Modified Brief Pain Inventory

9. Opioid-related symptom distress questionnaire

10. Antiemetic drugs administered

At the 48 hour assessment by a blinded observer:

1. Maternal satisfaction with the analgesia provided (NRS 0-10)

2. The occurrence of any post-operative urinary retention 
3. The occurrence of any respiratory depression (defined as a respiratory rate of less than 8 breaths per min, or a sedation score of 3 (difficult to rouse))

Post-operatively all women were monitored for respiratory depression by means of an hourly assessment of respiratory rate and sedation during the first $12 \mathrm{~h}$ postoperatively, and then at 4 hourly intervals until $48 \mathrm{~h}$.

\subsubsection{Sample Size Calculation}

The primary endpoint for the study was defined as the AUC for dynamic pain over the first 24 hours. Prior to this study there was only one previous study that had examined oral oxycodone in a treatment arm similar to this study (Davis, Esposito et al. 2006). For the purposes of clinical superiority of intrathecal morphine over oral oxycodone, a 30\% reduction in pain scores in the intrathecal morphine group was defined as a clinically significant reduction in pain scores. Prior to this study we had no data on the expected AUC pain scores, and hence the study had to be powered on the pain scores at individual time points. AUC is more representative of the overall pain experienced over a set time period, in comparison to pain scores at individual time points (Cappelleri, Bushmakin et al. 2009). The underlying assumption when using AUC pain measurements is that they should have greater sensitivity at detecting differences in the pain experienced. But, given the nature of measurement of AUC pain scores, what constitutes a clinically significant reduction in AUC pain scores has yet to be defined.

With this in mind, based on the data of Davis et al with an observed mean (SD) pain scores of $3.2(1.8)$ associated with the oral oxycodone regimen, and allowing for dropouts, a sample size of 120 (60 per group) was estimated. This gave an $80 \%$ power to detect a $30 \%$ difference in dynamic pain scores at the 12 hour postoperative assessment with an alpha of 0.05 (Power and Sample Size Program for Windows, PASS 2005, Kaysville Utah).

\subsubsection{Statistical Methods}

Descriptive statistics were summarised using median, interquartile range (IQR) and range and categorical outcomes were summarized using frequency distributions. The MBPI data were highly skewed so were analysed in discrete categories of score 0-3 (mild pain) or 4-10 (moderate to severe pain). The $\chi^{2}$ test or Fisher exact test 
was used for comparisons of categorical outcomes between groups. Univariate comparison of continuous outcomes between groups was performed using MannWhitney tests.

\section{$\underline{\text { AUC of pain scores }}$}

The AUC summaries were obtained using the linear trapezoidal rule form time 0 until the last pain intensity measured (Akhtar-Danesh 2001). The normality of the pain measurements and the baseline scores were tested using the Smirnov-

Kolmogorov test. The analysis of baseline pain scores was carried out using the ACNOVA with the initial pain recorded as a covariate. AUC values were transformed to natural logarithms to correct non-normality and weighted in the regression analysis to account for missing data at some time points. $P$ values $<0.05$ were considered statistically significant. The analysis was performed using SPSS 15.0 for Windows (SPSS, Chicago, IL).

Definition of cumulative AUC (Akhtar-Danesh 2001):

$$
\operatorname{AUC}(0, \mathrm{t})=\sum_{\mathrm{i}=0}^{\mathrm{t}-1}\left(\mathrm{t}_{\mathrm{i}+1}-\mathrm{t}_{\mathrm{i}}\right)\left(\mathrm{C}_{\mathrm{i}+1}-\mathrm{C}_{\mathrm{i}}\right) / 2
$$

\subsubsection{Ethical issues}

The National Health and Medical Research Council (NHMRC) and the Australian Research Council (ARC) publish a joint national statement on Ethical Conduct in Human Research and this was most recently updated in 2007 (National Health and Medical Research Council, Australian Research Council et al. 2009). This document provides a framework for researchers as well as reviewers upon which to identify and assess the ethical issues associated with research proposals. Of particular relevance are the guidelines on the Values and Principles of Ethical Conduct in human research. These guidelines are based around the four interrelated themes of justification, justice, beneficence and respect. Also of relevance in this document are the guidelines on the assessment and management of potential risks (in this case post-operative pain) and benefits (improved post-operative pain and side effect profile) in research.

There were two major ethical issues that were identified prior to the submission of this project to the Institutional Ethics Committee. The issues were that we 
proposed the introduction of a relatively untested method of post-operative pain relief and that this method of pain relief was a significant deviation from the usual method of post-caesarean section pain relief in this institution. These two issues as well as other issues related to the four interrelated themes are discussed in more detail below.

\subsubsection{Justification:}

This project underwent significant peer review as part of the grant application process prior to obtaining ethical approval. In this regard, in accordance with guideline 1.2 of the NHMRC statement, its research merit was not necessarily subject to the judgement of those ethically reviewing the work at the local institutional level. The project had the potential to benefit a large number of patients, both in and outside of Australia, which is also relevant in terms of the priorities of the NHMRC. In terms of the integrity of the project, this is related to both the project itself as well as the team conducting the research. In this regard the research was being performed by a research unit with a long history of performing high quality clinical research.

\subsubsection{Justice:}

In regards to the NHMRC guidelines on Justice in Research the overriding theme is one of fairness. The inclusion and exclusion criteria are such that most women presenting for elective caesarean section are eligible for recruitment into the study, improving both the internal and external validity of the study. At the time of the study it was the only study being performed on this patient population in this institution, meaning that there was no unfair burden of participation being placed upon this group of women. The results of this study have been published in the international literature as well as being presented at major scientific meetings which has given fair access to the results and to the benefits of the research to the wider community.

\subsubsection{Beneficence:}

Beneficence is the process of "assessing and taking account of the risk of harm and potential benefits....to participants" and "in being sensitive to the welfare and interests of people involved in their research". In relation to the conduct of this study, the core value of beneficence is especially important in that an as yet untested 
technique for post-operative pain management was being studied. Given that postoperative pain, by definition, is unpleasant and has the potential to cause harm, it was important to design the study so that if the post-operative pain was not well controlled, easily accessible and rapidly acting alternatives would be provided.

To achieve this two strategies were employed. Firstly, multimodal analgesia was used as background pain relief by means of an anti-inflammatory analgesic (parecoxib in the operating theatre and diclofenac post-operatively) and regular paracetamol. In addition to this, if participants were not experiencing satisfactory pain relief (as defined by both pain scores recorded and individual patient expectations), then a series of escalating analgesic options were made immediately available, with close support being provided from the research team and the acute pain service.

As part of the consent process for this study and in the patient information sheet it was made clear that patients may not benefit from being part of the study and that potentially they had a risk of having pain relief that was inferior to the well studied control technique (intrathecal morphine). It was made clear to patients the options that were available to them should their pain relief not be satisfactory and that they could also withdraw from the study at any point with no detriment to the care they were provided.

\subsubsection{Respect:}

Respect for human beings in research is the final core value in the NHMRC guidelines and is intrinsically linked to the values of justification, justice and beneficence. In relation to this study, respect is important in maintaining the privacy and confidentiality of the trial participants and also in ensuring that potential participants are empowered to make their own decisions in regards to participation in the research. In this regard a number of procedures are in place. All patient information is kept in a locked, secure environment within the hospital. Data is deidentified before being entered electronically into the database software.

Information about the trial is sent to eligible patients when their caesarean section is booked. This information contains a covering letter briefly introducing the trial and explaining that participation is entirely voluntary and that non participation will not in any way affect the care that is provided to them. Also included is the patient 
information sheet for the trial which contains contact numbers for the investigators should they have any questions.

When patients are next assessed prior to surgery (in the preadmission clinic) they are asked whether they have received the mail out and whether being involved in the study is of interest to them. Further detailed discussions are held and it is reinforced to patients that participation is voluntary and that they can withdraw from the study at any time if they wish with no impact on their care.

\subsubsection{Research Governance:}

Section 5 of the NHMRC Guidelines on Human Research as well as the Australian Code for the Responsible Conduct of Research describe the processes of research governance that should be in place for the ethical conduct of research. The principles of research governance are closely related and intertwined with the values described above. In addition, they provide guidelines that govern the ethical review process, the handling of complaints, the declaration and management of conflicts of interest and the overall institutional responsibilities for research conducted. The project and unit have no conflicts of interest in the research. The researchers send regular updates to the local ethics committee and any changes to the trial protocol are first put to the committee for approval. A complaints procedure for actual or potential participants is in place and details on how to access this are included in the original patient information sheet.

\subsubsection{Funding sources and budget}

The major costs associated with the conduct of this study related to the research personnel required for patient recruitment and data entry, as well as the pharmacy costs associated with the medications involved in the study. It was anticipated from previous studies performed at this institution that for each patient recruited who completes the study, approximately six hours of research nurse time would be required. This includes time for the screening for eligible patients, the sending out of patient information sheets, data collection and entry and liaison with pharmacy.

The pharmacy costs associated with this study included a set up fee which involved a quality assurance process to ensure that the intrathecal morphine prepared by the pharmacy was sterile and safe for intrathecal administration. The cost per patient 
included the expenses associated with the creation of dummy/placebo medication as well as the encapsulation of the medications to ensure appropriate blinding.

This budget can be heavily criticised in light of a number of lessons learnt during the Master of Clinical Research lecture series. The only direct staff costs that were accounted for are the time costs associated with the research nurses who are employed for data collection and analysis. No allowance was made for the research fellow(s) who will be involved in patient recruitment and staff education or for the statistical analysis of the data gathered. The travel costs associated with the presentation of the results of the study were also not included in the budget.

In defense of the budget presented, in terms of the priorities for research funding with the NHMRC, while having potential impact on a large number of Australian women, this project would be unlikely to rate highly in the grant review process. Traditional research agencies are unlikely to fund an expensive project of this nature. Agencies which are more closely involved with funding anaesthesia research, such as the Australian Society of Anaesthetists and the Australian and New Zealand College of Anaesthetists (ANZCA) have limited funds available and hence a balance has to be obtained to increase the likelihood of a successful grant. This does raise an issue which was reinforced during the lecture series. For a research unit and project to be successful funding for all members of staff involved is theoretically required. Research is less likely to be successful if the funding allocated to it is not sufficient. For the research unit in which this project was conducted, the funds received for research projects are kept in a general pool to pay for the costs of all staff involved. Further income is also generated through various teaching commitments through the department and this enables the research unit to run at a modest surplus each year.

Funding for the study, based on the budget presented below, was sought through two agencies. Funding was awarded by ANZCA for the entire amount displayed below with the requirement that it be completed in time to be presented at a plenary session at the Annual Scientific Meeting of the College in May 2008. 
Table 2: Initial budget

Personnel Research Midwife 12 hrs per week at $\$ 29.75$ per hour $\$ 22277$ plus $20 \%$ on costs

Pharmacy Set up and close out costs

Pharmacy Costs per patient $(\$ 50 \times 120)$

Stationary

For completeness, a budget has been generated based on the budget calculator in use at the University of Western Australia. This budget includes time for a research fellow, statistician and research nurse, pharmacy costs and 15\% indirect costs as per the UWA guidelines.

Table 3: Revised budget

\section{Budget Costing}

(should be quoted exclusive of GST)

\begin{tabular}{|c|c|c|}
\hline \multirow{3}{*}{$\begin{array}{c}\text { PeopleSoft } \\
\text { Account } \\
\text { Code }\end{array}$} & Year & 1 \\
\hline & Source of Funds & Funding Agency \\
\hline & Direct Costs & \\
\hline 500 & Academic Personnel (Salaries + On-Costs) & $\$ 26,532$ \\
\hline 520 & General Personnel ( Salaries + On-Costs) & $\$ 58,876$ \\
\hline \multirow[t]{2}{*}{$530 / 532$} & \multirow[t]{2}{*}{ Personnel (eg Stipends, Consultants) } & $\$ 0$ \\
\hline & & $\$ 85,408$ \\
\hline 854 & Equipment & $\$ 6,000$ \\
\hline 756 & Maintenance & $\$ 0$ \\
\hline 600 & Travel & $\$ 2,000$ \\
\hline \multirow[t]{2}{*}{640} & Animal Costs & $\$ 0$ \\
\hline & Other & $\$ 1,300$ \\
\hline \multirow[t]{2}{*}{497} & $15 \%$ Indirect Costs & $\$ 14,206$ \\
\hline & Total Costs & $\$ 108,915$ \\
\hline
\end{tabular}




\subsection{Results}

\subsubsection{Study Population}

One hundred and twenty patients were recruited to the study. Nine patients withdrew their consent to participate after commencement and were hence excluded from analyses (Figure 14). Of the nine patients excluded from the analyses, three withdrew consent for the study shortly after receiving the intrathecal study solution (Group $\mathrm{O} n=2$, Group $\mathrm{I} n=1$ ) and one patient required conversion to general anaesthesia (Group O). Four patients (Group I n=3, Group O n=1) withdrew their consent because of uncontrolled pain in the recovery room and one patient (Group O) withdrew within the first six hours because of pain.

One hundred and eleven patients with complete data (group $\mathrm{O} n=55$, group I $\mathrm{n}=56$ ) were included in the analysis. There were no major protocol violations among this cohort. The patients excluded did not differ significantly from the analysed study patients with respect to age, weight, height, QoR score, parity, ASA status or group randomisation. However, the pre-operative symptom distress scale (an evaluation of anxiety and concerns) of all subjects who withdrew from the study was significantly higher than those who remained in the study (median score 6 (IQR 4-10) in withdrawn subjects, median score 3 (IQR 1-5) in those who remained in the study, $P=0.019)$.

\subsubsection{Baseline demographics}

The characteristics of the two patient groups were similar, as shown in Table 4 below. There were no significant differences in any of the baseline characteristics between groups. 
Table 4: Baseline characteristics

\begin{tabular}{lllr}
\hline & $\begin{array}{l}\text { Group I } \\
\text { (intrathecal morphine) } \\
(\mathbf{n = 5 6 )}\end{array}$ & $\begin{array}{l}\text { Group O } \\
\text { (oral oxycodone) } \\
(\mathbf{n = 5 6 )}\end{array}$ & p-value \\
\hline Age (y) & $32(28-37 ; 20-44)$ & $31(26-36 ; 20-42)$ & 0.311 \\
Weight (kg) & $86(73-107 ; 58-153)$ & $87(76-107 ; 51-157)$ & 0.864 \\
Height (cm) & $165(162-170 ; 152-183)$ & $165(158-169 ; 152-183)$ & 0.666 \\
Parity & $2(1-2 ; 0-4)$ & $1(1-2 ; 0-4)$ & 0.345 \\
ASA=2 & $21(38 \%)$ & $24(43 \%)$ & 0.561 \\
Previous CS & $1(1-2 ; 0-4)$ & $1(0-1 ; 0-3)$ & 0.194 \\
Pre-op QOR score & $17(15-18 ; 10-18)$ & $16(15-17 ; 9-18)$ & 0.621 \\
(range 0-18) & $3(2-5 ; 0-16)$ & $3(1-5 ; 0-14)$ & 0.684 \\
$\begin{array}{l}\text { Pre-op SDQ } \\
\text { (range 0-36) }\end{array}$ & & & \\
\hline
\end{tabular}

$\mathrm{N} \%$ or median, (IQR; R) are shown, as appropriate. 
Figure 14: Participant flow through the trial

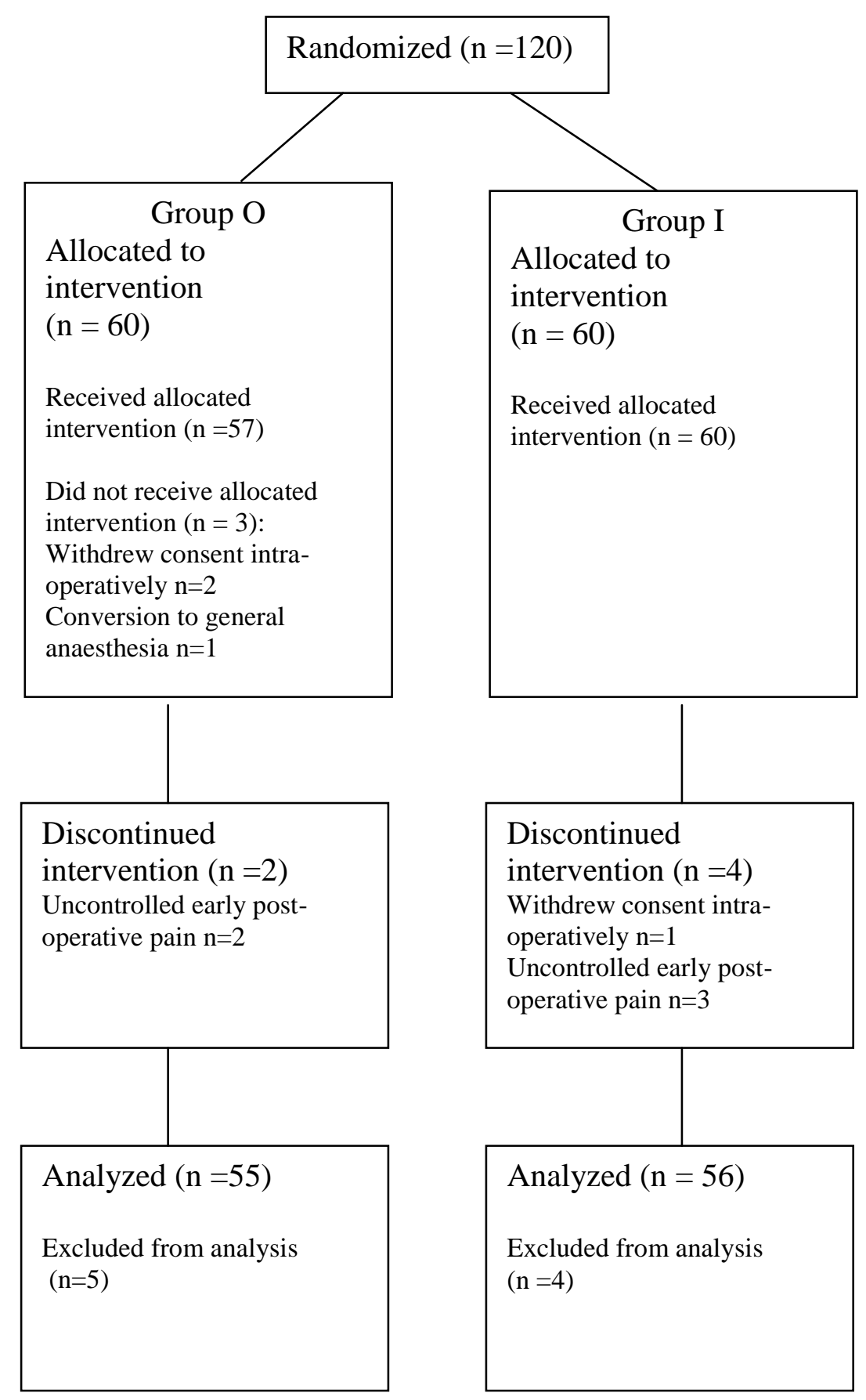




\subsubsection{Primary and Secondary Endpoints}

\subsubsection{Primary Endpoint}

The 0-24 AUC pain scores with movement did not differ significantly between groups (63[IQR 37-96] vs. 71[IQR 42-99] in Groups I and O respectively, $\mathrm{P}=0.319$ ) (Figure 15, Table 5). The AUC pain score did not differ significantly with simultaneous adjustment for the use of post-operative oxycodone ( $P$ values $=0.53)$. Based on a post hoc power analysis, the study had $95 \%$ power to detect a $30 \%$ difference in 24 -h AUC pain scores between groups and $86 \%$ power to show a $25 \%$ difference between groups.

Figure 15: AUC scores for pain with rest and movement and sedation scores

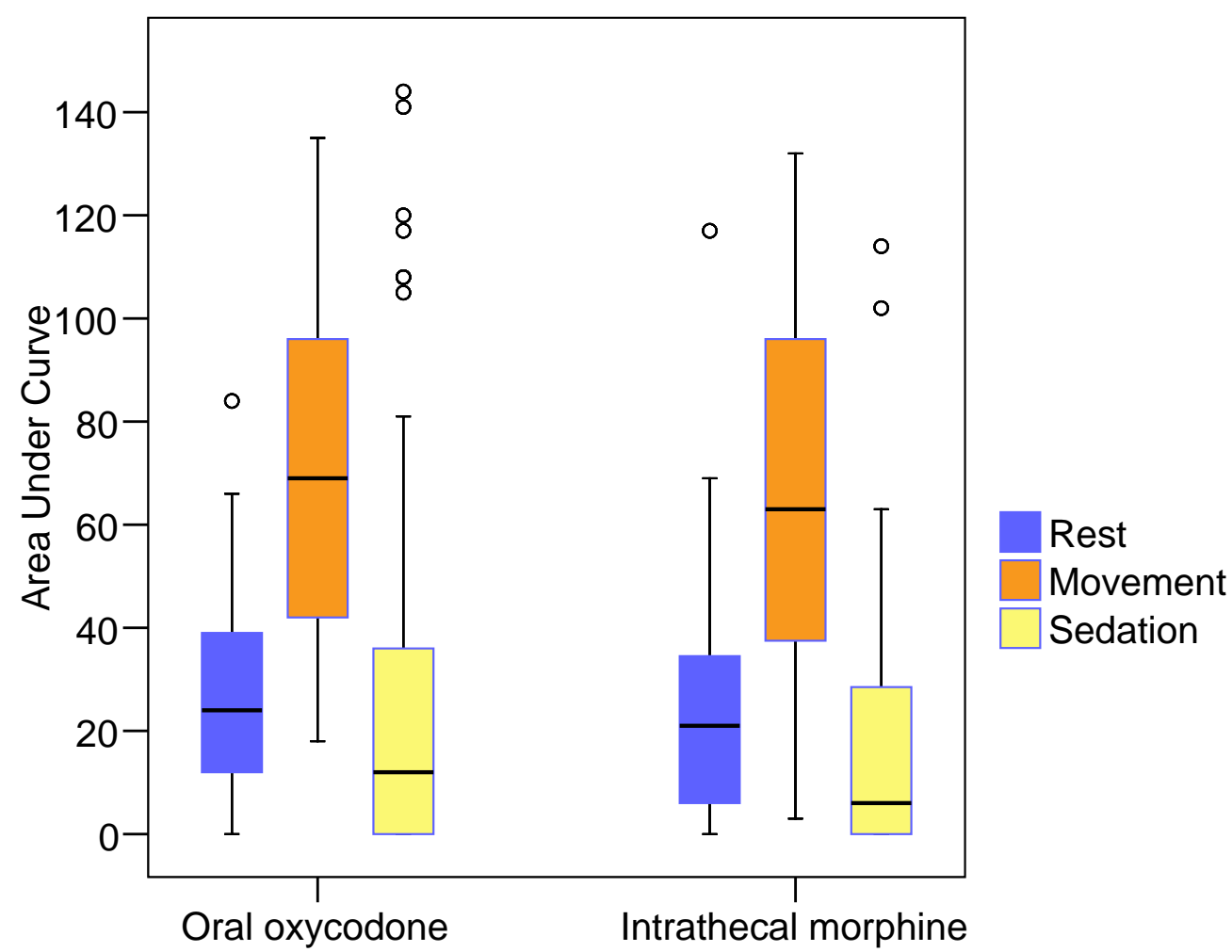

Note: Two cases in Group $\mathrm{O}$ had 3 missing datapoints; 1 case had missing data at 12, 18 and 24 hours for rest, movement and sedation and 1 case in Group $\mathrm{O}$ had missing movement data for timepoints 6, 12, and 18 hours. These 2 cases did not have AUC calculated for relevant areas.

In Group O, 49 cases had complete data for rest AUC, 48 cases for movement AUC and 49 cases for sedation AUC. In Group I, 52 cases has complete data for rest and movement AUC and 51 cases for sedation AUC. 


\subsubsection{Secondary Endpoints}

\subsection{VAS, AUC scores for pain and sedation}

The 0-24 h AUC scores for pain at rest and sedation did not differ significantly between groups (Figure 15, Table 5). VAS pain scores at rest were low in both groups at all time points (Figure 16, Table 5), although at $12 \mathrm{~h}$ the median pain score at rest in group $\mathrm{O}$ was higher than that in group I (2 [IQR 1-3] vs. 1 [IQR 02]; $P=0.03)$. There was no significant difference between groups for pain scores with movement at any time point, although there was a trend towards higher early post-operative pain scores in Group O, with a median pain score of 5[IQR 3-7] vs $4[\mathrm{IQR} 3-6](\mathrm{P}=0.062)$ in Groups $\mathrm{O}$ and I respectively (Figure 17). Sedation scores were low and similar in both groups and not significantly different, although there was a trend towards more sedation at $18 \mathrm{~h}$ in Group $\mathrm{O}(\mathrm{P}=0.057)$ (Table 5, Figure 18).

Figure 16: VAS pain scores with rest

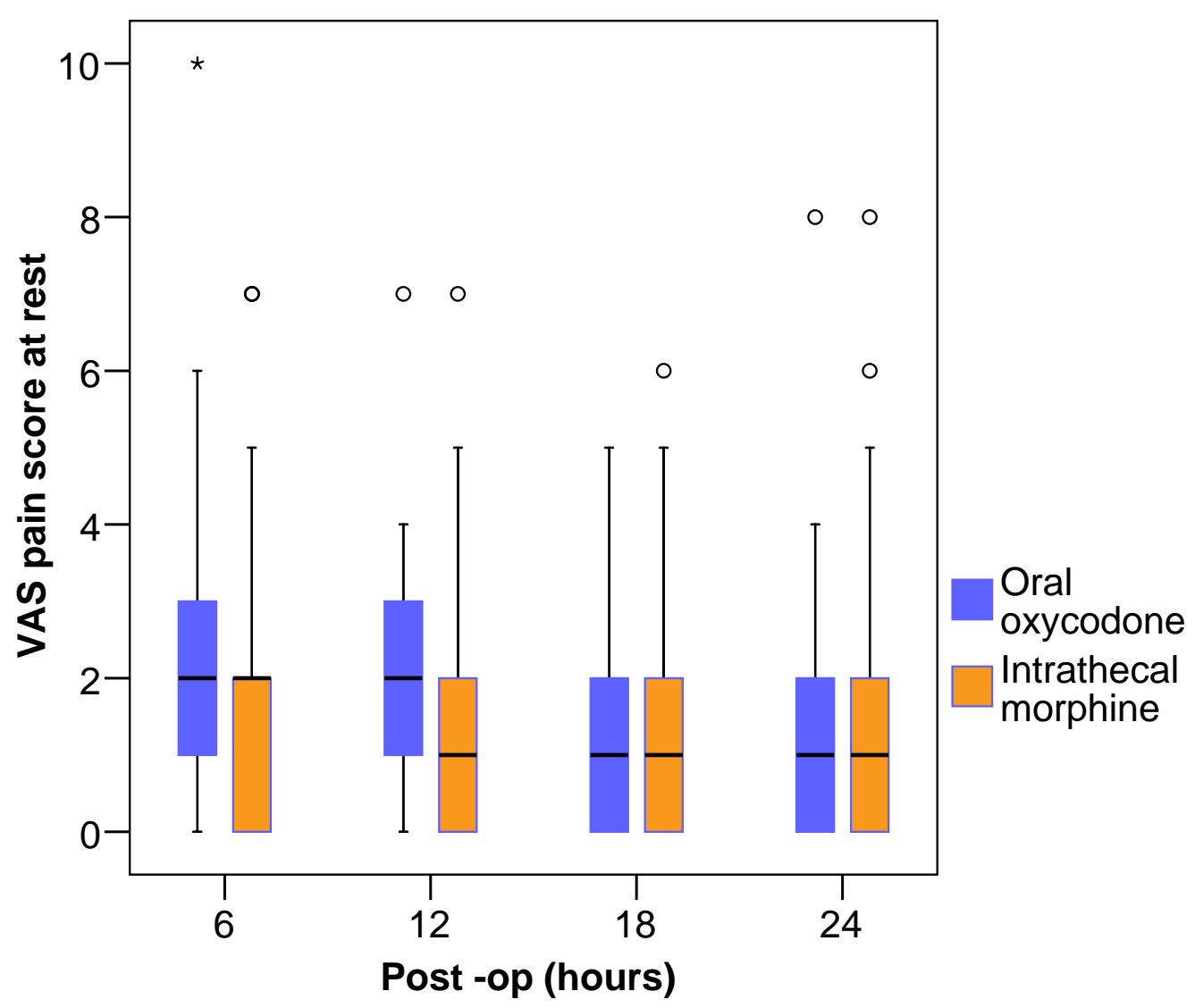


Figure 17: VAS Pain scores with movement

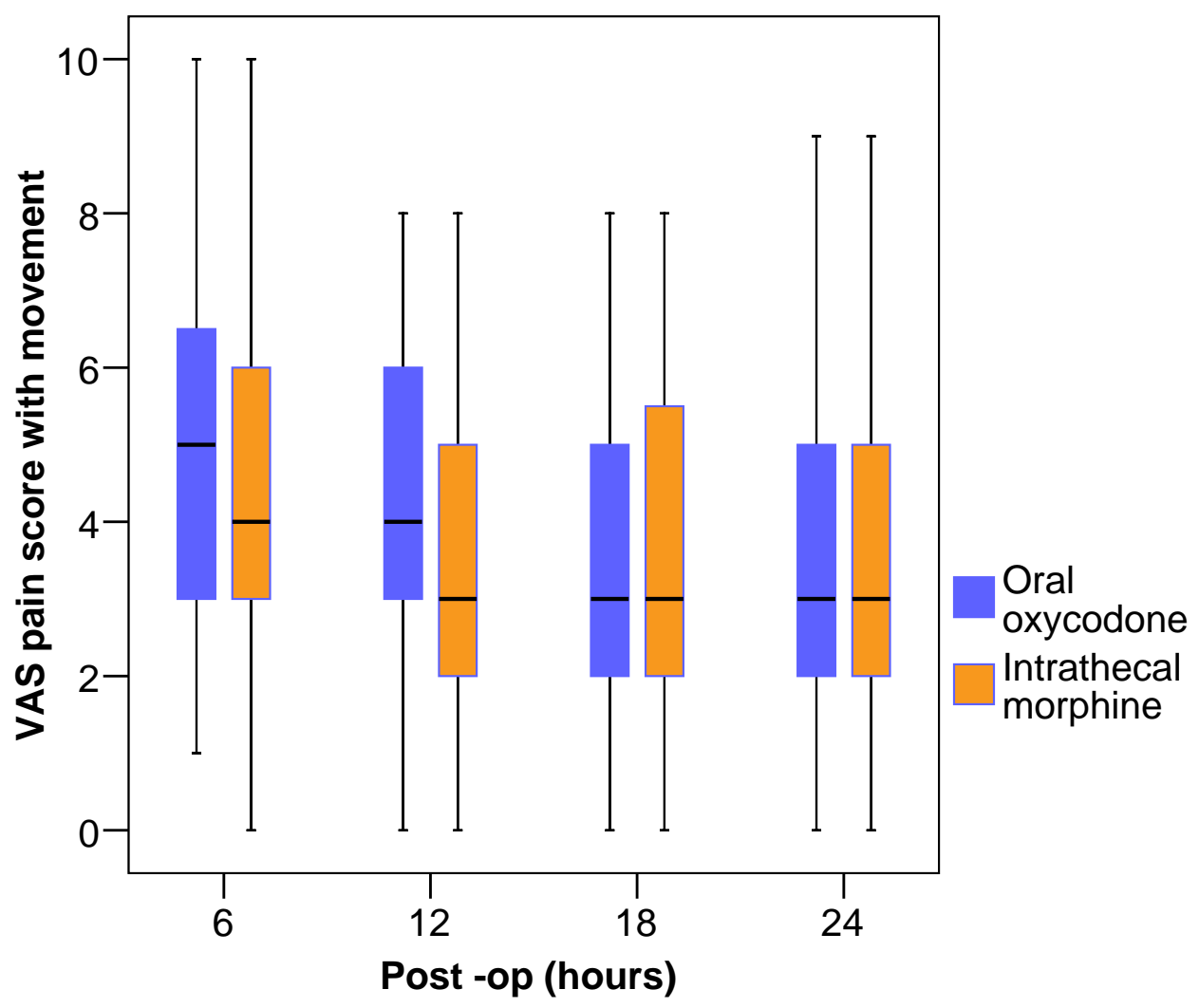

Figure 18: VAS sedation scores

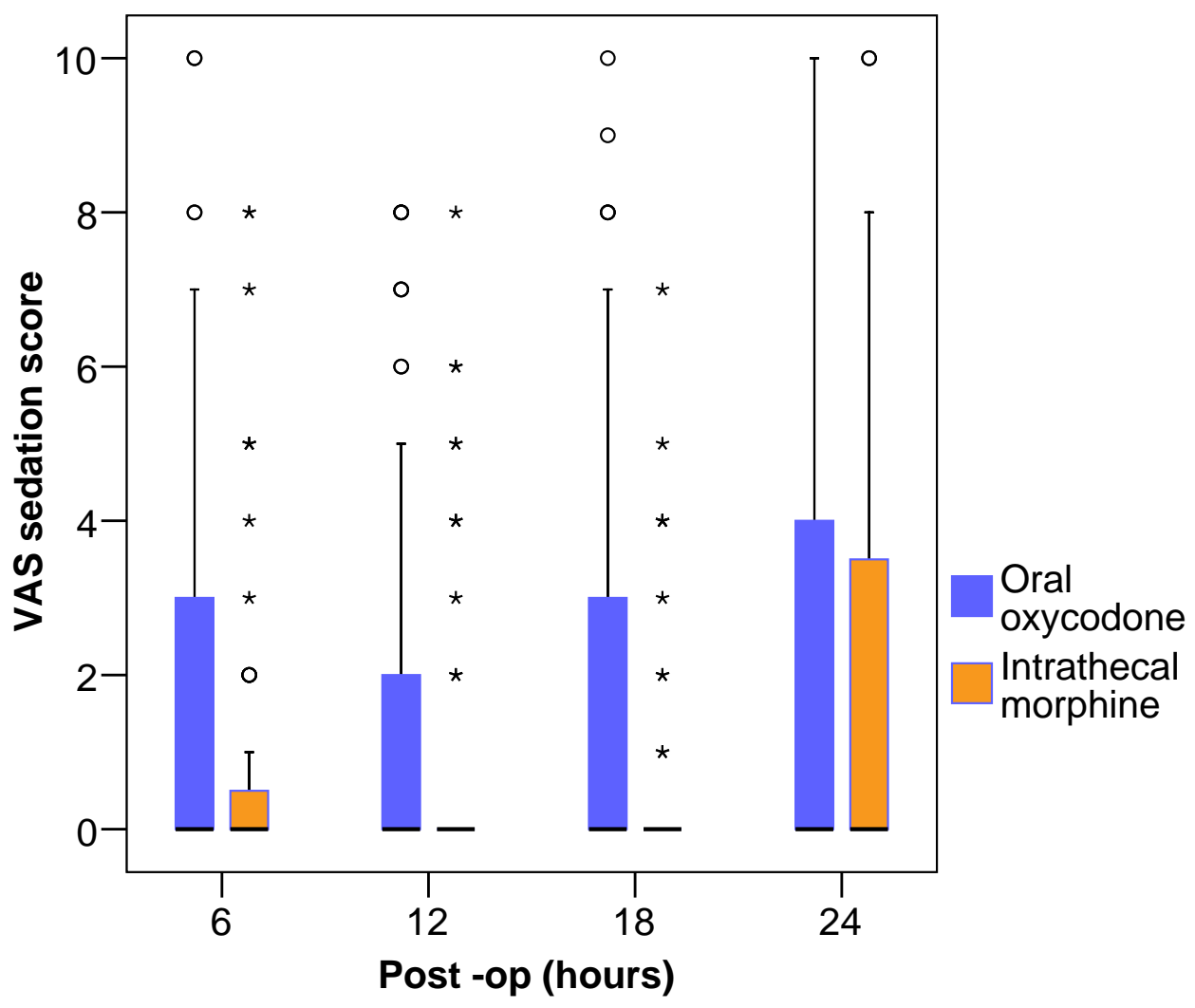


Table 5: VAS and AUC results for pain and sedation

\begin{tabular}{|c|c|c|c|}
\hline & $\begin{array}{l}\text { Group I } \\
\text { (intrathecal morphine) } \\
(n=55)\end{array}$ & $\begin{array}{l}\text { Group } O \\
\text { (oral oxycodone) } \\
(n=56)\end{array}$ & p-value \\
\hline \multicolumn{4}{|l|}{ VAS pain scores } \\
\hline \multicolumn{4}{|l|}{ At rest } \\
\hline 6 hours & $2(0-3 ; 0-7)$ & $2(1-3 ; 0-10)$ & 0.178 \\
\hline 12 hours & $1(0-2 ; 0-7)$ & $2(1-3 ; 0-7)$ & 0.030 \\
\hline 18 hours & $1(0-2 ; 0-6)$ & $1(0-2 ; 0-5)$ & 0.589 \\
\hline 24 hours & $1(0-2 ; 0-8)$ & $1(0-2 ; 0-8)$ & 0.688 \\
\hline \multicolumn{4}{|l|}{ With movement } \\
\hline 6 hours & $4(3-6 ; 0-10)$ & $5(3-7 ; 1-10)$ & 0.062 \\
\hline 12 hours & $3(2-5 ; 0-8)$ & $4(3-6 ; 0-8)$ & 0.100 \\
\hline 18 hours & $3(2-6 ; 0-8)$ & $3(2-5 ; 0-8)$ & 0.852 \\
\hline 24 hours & $3(2-5 ; 0-9)$ & $3(2-5 ; 0-9)$ & 0.792 \\
\hline \multicolumn{4}{|l|}{ Sedation score } \\
\hline 6 hours & $0(0-1 ; 0-8)$ & $0(0-3 ; 0-7)$ & 0.309 \\
\hline 12 hours & $0(0-0 ; 0-8)$ & $0(0-3 ; 0-8)$ & 0.092 \\
\hline 18 hours & $0(0-3 ; 0-7)$ & $0(0-3 ; 0-10)$ & 0.057 \\
\hline 24 hours & $0(0-4 ; 0-10)$ & $0(0-4 ; 0-10)$ & 0.826 \\
\hline \multicolumn{4}{|l|}{ AUC to 24 hours } \\
\hline rest & $21(6-35 ; 0-117)$ & 24 (12-39; 0-84) & 0.306 \\
\hline movement & $63(37-96 ; 3-132)$ & $71(42-99 ; 18-135)$ & 0.319 \\
\hline sedation & $6(0-29 ; 0-114)$ & $12(0-42 ; 0-144)$ & 0.237 \\
\hline $\begin{array}{l}\text { Time to first on- } \\
\text { demand oxycodone } \\
\text { request (mins) }\end{array}$ & 160 (92-230; 23-1419) & $144(64-218 ; 20-1440)$ & 0.597 \\
\hline $\begin{array}{l}\text { Additional analgesia } \\
\text { (mg) }\end{array}$ & $35(63 \%)$ & $46(82 \%)$ & 0.034 \\
\hline $\begin{array}{l}\text { Oxycodone } \\
\left(\mathrm{n}_{\mathrm{I}}=32, \mathrm{n}_{\mathrm{O}}=44\right)\end{array}$ & $25(10-35 ; 5-70)$ & $25(10-44 ; 5-85)$ & 0.953 \\
\hline $\begin{array}{l}\text { Tramadol } \\
\left(\mathrm{n}_{\mathrm{I}}=19, \mathrm{n}_{\mathrm{O}}=29\right)\end{array}$ & $100(100-100 ; 50-300)$ & $100(100-200 ; 50-500)$ & 0.299 \\
\hline
\end{tabular}

Median, (IQR; R) are shown 


\subsection{Supplemental Analgesia}

There was no significant difference in the number of patients needing intraoperative supplementation, with 13 patients in group I (23\%) and 9 in group O (16\%) requiring supplementation ( $P=0.37)$. The median time to the first post-operative request for supplemental analgesia was 160[IQR 92-230] min for Group I and 144[IQR 62-218] min for Group O ( $\mathrm{P}=0.597)$. Overall additional analgesia was more likely to be requested by patients in Group O ( $82 \%$ vs $63 \%, \mathrm{P}=0.034)$. Of the women in Group $\mathrm{O}$ requesting additional supplementation, the median dose requirements of oxycodone were $25 \mathrm{mg}$ over 24 hours, although some women required considerably higher doses, with the range being 5-85 mg [IQR 10-35]. The median dose of oxycodone in Group I was the same at $25 \mathrm{mg}$, with the range also showing that some women required considerable amounts of additional analgesia (range 5-70 mg, IQR [10-44]) (Table 5). The pain scores for individual patients through the trial, commencing with their pain scores at rest and movement at the 6 h mark, are shown in Figure 19 and 20. 
Figure 19: Subject profile plots for pain scores at rest (NRS)

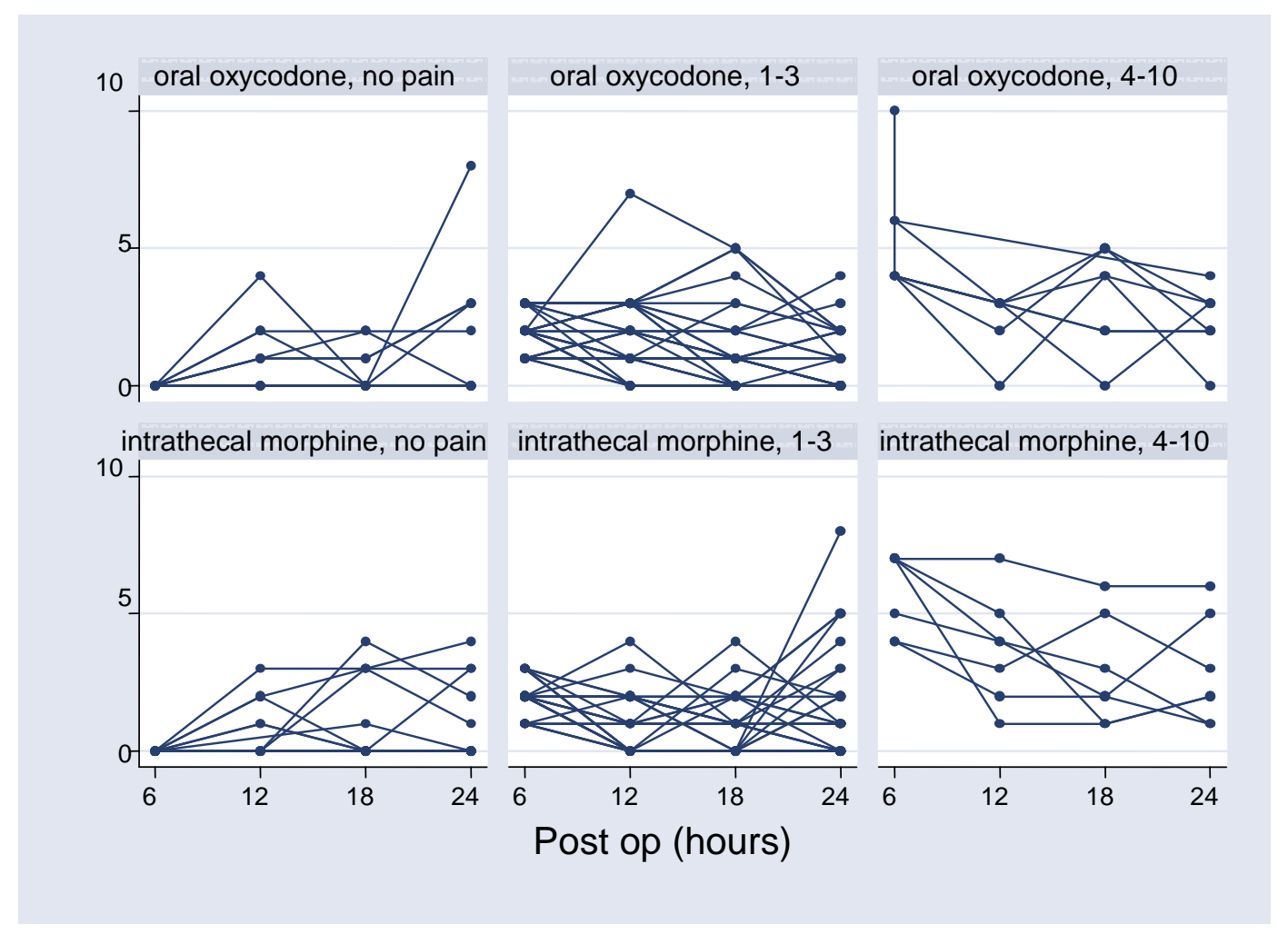

Note: 0 - no pain, 1-3-moderate pain, 4-10 -severe pain 
Figure 20: Subject profile plots for pain scores with movement (NRS)
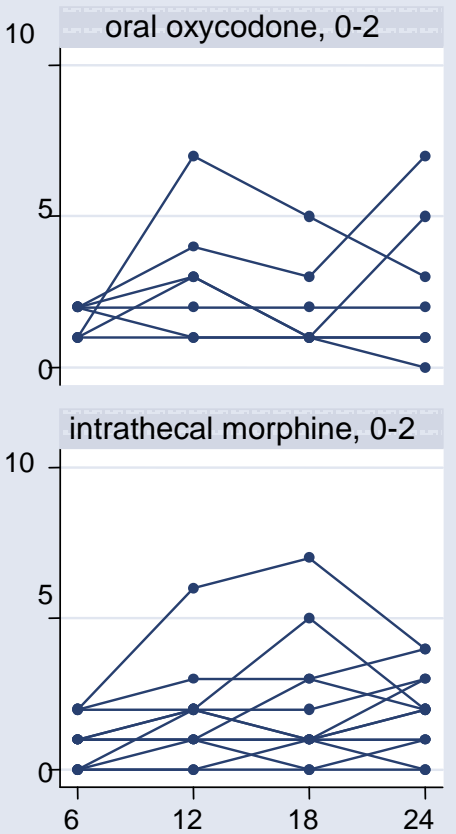

oral oxycodone, 3-6

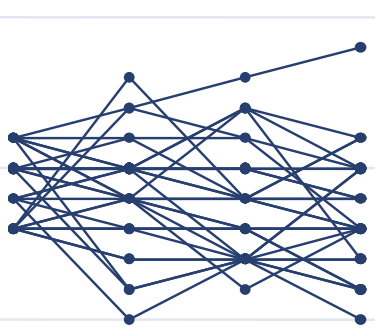

intrathecal morphine, 3-6

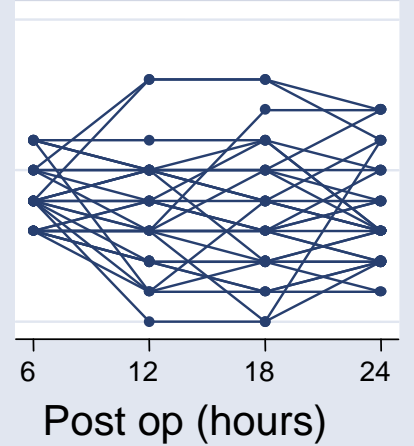

oral oxycodone, 7-10

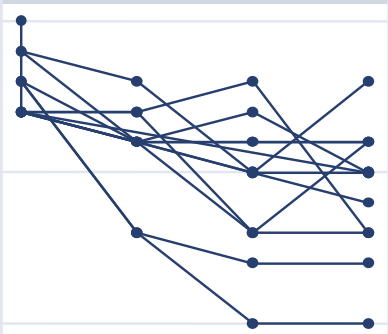

intrathecal morphine, 7-10
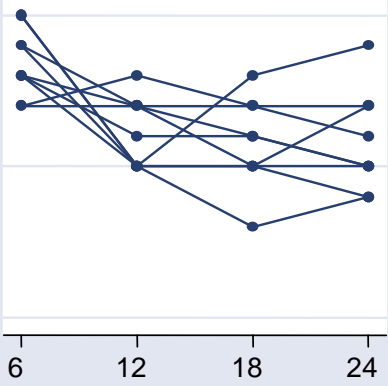

Note: 0-2 -mild pain, 3-6-mod pain, 4-10-severe pain 


\subsection{Modified Brief Pain Inventory Data}

Total scores for the MBPI did not differ between the groups. More patients in Group O than in Group I rated their worst pain experienced 4-10/10 during the first 24 h post-operatively ( $87 \%$ vs. $64 \% ; P=0.007$ ) (Table 6 ).

Table 6: Modified Brief Pain Inventory data

\begin{tabular}{|c|c|c|c|c|}
\hline & & Group I $(n=55)$ & Group $O(n=56)$ & p-value \\
\hline & & $\mathbf{N}(\%)$ & $\mathrm{N}(\%)$ & \\
\hline \multicolumn{5}{|l|}{ MBPI-SF } \\
\hline \multicolumn{2}{|l|}{ Any pain last $24 \mathrm{hr}$} & $33(59 \%)$ & $39(70 \%)$ & 0.234 \\
\hline \multirow[t]{2}{*}{ Worst pain $24 \mathrm{hr}$} & $0-3$ & $20(36 \%)$ & $7(13 \%)$ & 0.007 \\
\hline & $4-10$ & $36(64 \%)$ & $48(87 \%)$ & \\
\hline \multirow[t]{2}{*}{ Least pain $24 \mathrm{hr}$} & $0-3$ & $52(93 \%)$ & $53(96 \%)$ & 0.679 \\
\hline & $4-10$ & $4(7 \%)$ & $2(4 \%)$ & \\
\hline \multirow[t]{2}{*}{ Average pain $24 \mathrm{hr}$} & $0-3$ & $38(68 \%)$ & $40(73 \%)$ & 0.679 \\
\hline & $4-10$ & $18(32 \%)$ & $15(27 \%)$ & \\
\hline \multirow[t]{2}{*}{ Pain now } & $0-3$ & $48(86 \%)$ & $50(91 \%)$ & 0.557 \\
\hline & $4-10$ & $8(14 \%)$ & $5(9 \%)$ & \\
\hline \multirow[t]{2}{*}{$\begin{array}{l}\text { Relief after medication } \\
(\%)\end{array}$} & $0-30 \%$ & $-(-)$ & $3(6 \%)$ & 0.112 \\
\hline & $40-100 \%$ & $56(100 \%)$ & $50(94 \%)$ & \\
\hline \multicolumn{5}{|l|}{ Pain interfering with: } \\
\hline \multirow[t]{2}{*}{ Activity } & $0-3$ & $31(56 \%)$ & $26(46 \%)$ & 0.445 \\
\hline & $4-10$ & $24(44 \%)$ & $29(53 \%)$ & \\
\hline \multirow[t]{2}{*}{ Mood } & $0-3$ & $51(91 \%)$ & $48(87 \%)$ & 0.557 \\
\hline & $4-10$ & $5(9 \%)$ & $7(13 \%)$ & \\
\hline \multirow[t]{2}{*}{ Walking } & $0-3$ & $25(45 \%)$ & $28(53 \%)$ & 0.564 \\
\hline & $4-10$ & $30(55 \%)$ & $25(47 \%)$ & \\
\hline \multirow[t]{2}{*}{ Relations with people } & $0-3$ & $37(66 \%)$ & $31(57 \%)$ & 0.433 \\
\hline & $4-10$ & $19(34 \%)$ & $23(43 \%)$ & \\
\hline \multirow[t]{2}{*}{ Sleep } & $0-3$ & $42(75 \%)$ & $39(71 \%)$ & 0.673 \\
\hline & $4-10$ & $14(25 \%)$ & $16(29 \%)$ & \\
\hline \multirow[t]{2}{*}{ Coughing } & $0-3$ & $29(52 \%)$ & $27(49 \%)$ & 0.850 \\
\hline & $4-10$ & $27(48 \%)$ & $28(51 \%)$ & \\
\hline \multirow[t]{2}{*}{ Deep breathing } & $0-3$ & $48(86 \%)$ & $51(93 \%)$ & 0.360 \\
\hline & $4-10$ & $8(14 \%)$ & $4(7 \%)$ & \\
\hline \multirow[t]{2}{*}{ Concentration } & $0-3$ & $51(91 \%)$ & $46(84 \%)$ & 0.265 \\
\hline & $4-10$ & $5(9 \%)$ & $9(16 \%)$ & \\
\hline
\end{tabular}




\subsection{Side effects and satisfaction}

There was a significantly higher incidence of pruritus in group I (87\% vs. 56\%, P $=0.001)$ and this was also more severe (median score $4[\mathrm{IQR} 2-5]$ vs. $1[\mathrm{IQR} 0-4]$ in groups I and $\mathrm{O}$ respectively, $\mathrm{P}=0.001$ ) (Table 7). There were no significant differences between groups for the incidence of nausea, epigastric pain or urinary retention. No respiratory depression (based on clinical observation of conscious state and ventilation) was observed in either group. At the $24 \mathrm{~h}$ review patients in group I had higher satisfaction scores (10[IQR 8-10] vs. 8[IQR 7-10], P=0.010) but this advantage was no longer present by $48 \mathrm{~h}$.

Table 7: Outcomes during recovery

\begin{tabular}{|c|c|c|c|}
\hline & $\begin{array}{l}\text { Group I } \\
\text { (intrathecal morphine) } \\
(\mathrm{n}=55)\end{array}$ & $\begin{array}{l}\text { Group } O \\
\text { (oral oxycodone) } \\
(n=56)\end{array}$ & p-value \\
\hline \multicolumn{4}{|l|}{24 hours post-op } \\
\hline Nausea & $26(46 \%)$ & $19(34 \%)$ & 0.247 \\
\hline Epigastric pain & $6(11 \%)$ & $3(5 \%)$ & 0.490 \\
\hline $\begin{array}{l}\text { Satisfaction with } \\
\text { analgesia }\end{array}$ & $10(8-10 ; 3-10)$ & $8(7-10 ; 2-10)$ & 0.010 \\
\hline \multicolumn{4}{|l|}{ Global score $(0-10)$} \\
\hline nausea & $1(0-3 ; 0-8)$ & $0(0-4 ; 0-9)$ & 0.469 \\
\hline sedation & $2(0-5 ; 0-9)$ & $3(0-6 ; 0-10)$ & 0.141 \\
\hline pruritis & $4(2-5 ; 0-9)$ & $1(0-4 ; 0-9)$ & 0.001 \\
\hline QOR score (0-18) & $15(13-17 ; 10-18)$ & $15(13-16 ; 9-18)$ & 0.377 \\
\hline $\begin{array}{l}\text { Difference QOR } \\
\text { (Post - pre ) } \\
\text { *+ve score shows } \\
\text { improvement }\end{array}$ & $-1(-3-0 ;-7-4)$ & $-2(-4-0 ;-7-5)$ & 0.371 \\
\hline SDQ $(0-36)$ & $7(3-10 ; 0-19)$ & $6(3-12 ; 0-18)$ & 0.806 \\
\hline $\begin{array}{l}\text { Difference SDQ } \\
\text { (Post - pre ) } \\
\text { *-ve score shows } \\
\text { improvement }\end{array}$ & $2(1-7 ;-6-13)$ & $3(0-8 ;-8-14)$ & 0.730 \\
\hline \multicolumn{4}{|l|}{ Antiemetics } \\
\hline ondanestron & $21(38 \%)$ & $13(24 \%)$ & 0.150 \\
\hline naloxone & $6(11 \%)$ & $4(7 \%)$ & 0.742 \\
\hline other & $6(11 \%)$ & $2(4 \%)$ & 0.271 \\
\hline \multicolumn{4}{|l|}{48 hours post-op } \\
\hline $\begin{array}{l}\text { Satisfaction with } \\
\text { analgesia }(0-10)\end{array}$ & $9(8-10 ; 2-10)$ & $9(8-9 ; 0-10)$ & 0.887 \\
\hline Urinary retention & - & $1(2 \%)$ & 1.000 \\
\hline $\begin{array}{l}\text { Respiratory depression } \\
(\mathrm{RR}<8 \text { or sedation } \\
\text { score }=3)\end{array}$ & - & - & - \\
\hline
\end{tabular}

$\mathrm{N} \%$ or median, (IQR; R) are shown, as appropriate. 


\subsection{Discussion}

\subsubsection{Summary}

In this randomised controlled trial, a multimodal regimen of post-caesarean analgesia that consisted of regular oral non opioid medication in conjunction with oxycodone provided satisfactory analgesia. It resulted in post-operative analgesia over the first 24 hours after surgery that was of a similar level to that seen with 100 mcg of intrathecal morphine, although pain at rest at 12 hours post-operatively was significantly higher in the oxycodone group. The intrathecal morphine regimen was associated with higher $24 \mathrm{~h}$ maternal satisfaction, despite a higher incidence of pruritus, although this difference in maternal satisfaction was no longer present at the $48 \mathrm{~h}$ review. Supplementation of the primary analgesia regimen was high in both groups, although more women in the oxycodone group required additional supplementation. The incidence and severity of nausea was not significantly different, although the 24 hour NRS score for nausea, the global score for nausea and antiemetic use were all lower in the oxycodone group.

\subsubsection{Oral analgesia post-caesarean delivery}

Multimodal analgesic regimens are commonly recommended for post-caesarean analgesia (Lavand'homme 2006; Pan 2006). These regimens are generally based on an opioid in combination with paracetamol and anti-inflammatory drugs. The oral route of administration of opioids is commonly used in a step-down or supplementary role, but has received little attention in the literature as the primary route of administration post-caesarean. Prior to this thesis, the author was aware of only one previous randomised controlled trial in this context. In that study, oral oxycodone was associated with improved pain relief and maternal satisfaction when compared with intravenous morphine (Davis, Esposito et al. 2006). Two cohort studies have also been performed (Jakobi, Weiner et al. 2000; Jakobi, Solt et al. 2002), but the design of these studies limit the conclusions that can be drawn. They demonstrated the potential feasibility of delivering an oral regimen, which, when combined with published correspondence and audit data, highlighted the need for a properly designed randomised trial in this area.

In the post-operative setting, especially post-caesarean delivery, early oral intake has raised concerns in regards to decreased bowel motility and increased post-operative 
nausea and vomiting (Jakobi, Weiner et al. 2000). Nevertheless early oral intake after caesarean delivery is acceptable to most women and has been associated with shorter lengths of hospital stay and earlier recovery of bowel function (Patolia, Hilliard et al. 2001). The oral route of administration avoids some of the issues associated with the rectal route, particularly the potential for unreliable drug absorption as well as the low patient acceptability of such an approach. In comparison to the intravenous route of administration, the oral route avoids some of the hazards such as the risk of device programming errors, device malfunctions and the rapid fluctuation in plasma concentrations that can occur with intravenous techniques. The advantages over the use of epidural and intrathecal opioids are the potential for decreased post-operative pruritus and nausea or vomiting and also the potential advantages for the mother of not having an epidural catheter in situ postoperatively.

In terms of the possible choices of oral opioid, oral oxycodone was chosen rather than oral morphine for this study. This was because it is the most popular oral opioid in Australia in this setting (Seaton, Reeves et al. 2007), and hence is more familiar to anaesthetists and other post natal staff. In Australia the cost of these two drug formulations is similar but oxycodone has more rapid and reliable absorption and kinetics, as well as having an established role in post-operative pain management (Riley, Eisenberg et al. 2008). The breast milk transfer of oxycodone is low and considered compatible with breast feeding, especially during the first day or two after delivery, when milk production is low (Seaton, Reeves et al. 2007). Oral morphine has not been adequately investigated in post-caesarean analgesia and its effect on the neonate has not been evaluated. Nevertheless, maternal plasma concentrations of opioid are likely to be significantly higher than with neuraxial opioid administration, which may need consideration in individual cases, especially when doses of more than $90 \mathrm{mg}$ per $24 \mathrm{~h}$ are used for several days (Seaton, Reeves et al. 2007).

The results from this study suggest that an oral opioid based regimen is acceptable to women post-caesarean delivery and adds a well designed clinical trial to the research on this topic. In contrast to the results of Davis et al, we did not show that an oral regimen resulted in improved post-operative pain relief or satisfaction. We believe that this was due to our choice of control group. Davis et al chose patient controlled IV morphine as their control group. This method is associated with a 
higher incidence of side effects and decreased satisfaction when compared with the neuraxial route of administration (Paech, Moore et al. 1994; Ngan Kee, Lam et al. 1997). In contrast, intrathecal morphine, despite not having the advantage of being a patient controlled technique, appears to result in more consistently effective analgesia and despite a significant level of pruritus, is generally associated with high levels of patient satisfaction (Sarvela, Halonen et al. 2002).

Interestingly, this is the first study that the author is aware of in which parecoxib has been used in women undergoing caesarean delivery. Parecoxib is in routine use for this indication in the institution in which this study was conducted and is currently the only intravenous COX-2 specific agent available in Australia. To date there has only been one post-caesarean study that has examined the use of COX-2 agents. This study investigated valdecoxib (an oral COX-2 agent and the active drug within parecoxib) but was stopped early as a consequence of emerging concerns in relation to the use of COX-2 agents and adverse cardiac events (Carvalho, Chu et al. 2006). We elected to continue to use parecoxib as it was administered as a single dose only to patients without significant risk factors for cardiac or cerebral events. It also has a prolonged analgesic effect (12-24 h) (Amabile and Spencer 2004) and a proven benefit when combined with intrathecal morphine in other situations (Niruthisard, Werawataganon et al. 2007). It may also reduce the risk of exacerbating post-partum blood loss (due to a lack of any affect on platelet function (Munsterhjelm, Niemi et al. 2006; Graff, Arabmotlagh et al. 2007)) or of causing hypertension compared with traditional NSAIDs. There is currently no data available on the safety of breast feeding and parecoxib, although a study is underway in the author's own institution to examine this further.

Previous studies have documented relatively high supplementation rates with additional opioid medication when intrathecal morphine is given. In the study by Duale et al, when $75 \mathrm{mcg}$ of intrathecal morphine was used, $88 \%$ of subjects required additional supplemental morphine (Duale, Frey et al. 2003). When Paech et al studied a $200 \mathrm{mcg}$ dose of intrathecal morphine, the supplementation rate was 76\% (Paech, Pavy et al. 2000). In this study, 63\% of the women in Group I requested additional opioid analgesia and whilst the interquartile range for the dose of additional oxycodone is relatively narrow $(10-35 \mathrm{mg})$, the overall range is 5-70 $\mathrm{mg}$, indicating that some women needed a comparatively high dose of additional opioid. Two points are worth noting from these findings. Firstly, the 
supplementation rate in this study is consistent with other studies that used a similar dose of intrathecal morphine, reinforcing the need to have additional opioid medication available for these women. Secondly, the findings are also consistent with those of Swart et al (Swart, Sewell et al. 1997). They found that when they administered 100 mcg of intrathecal morphine, followed by patient controlled IV morphine analgesia, over $50 \%$ of patients used less than $10 \mathrm{mg}$ of morphine in the first 24 hours. But in contrast more than 10\% required more than $40 \mathrm{mg}$ over the same period, demonstrating that whilst intrathecal morphine is effective for most individuals, it is relatively ineffective in a small but significant portion of the population (such as the patient in this study that required $70 \mathrm{mg}$ of supplemental oxycodone).

In this study, the oral oxycodone regimen produced satisfactory analgesia, confirming the efficacy of such an approach. However, at $24 \mathrm{~h}$ the maternal satisfaction was higher in the intrathecal group, which contrasts with previous studies using an oral regimen (although none have used intrathecal morphine as the control group) (Davis, Esposito et al. 2006). This was despite a favourable side effect profile for the oral oxycodone, especially in regards to a lower incidence of pruritus in the oxycodone group. A potential explanations for lower satisfaction in the oxycodone group may lie in the peaks and troughs in analgesic effect provided by an intermittent dosing method, in comparison to what is likely to be a more consistent level of analgesia with intrathecal morphine, which maintains analgesic cerebrospinal fluid concentrations for up to 24 hours (Chaney 1995). This is supported by data from some of the secondary endpoints in the study. Data from the Modified Brief Pain Inventory indicates that women in the oral oxycodone group were more likely to report their worst pain as being of a higher intensity (Table 6). This suggests that post-caesarean patients consider consistently good pain relief more important than side effects such as pruritus.

Opioid related side effects are partly determined by the dose and route of opioid administration (Fishman, Condon et al. 2004). In this study the major side effects examined were pruritus, nausea and sedation. A dose of $100 \mathrm{mcg}$ of intrathecal morphine was chosen in this study as it appears to provide the optimal balance between analgesia and side effects (Palmer, Emerson et al. 1999). Increasing the dose above $100 \mathrm{mcg}$ appears to have little benefits in terms of the analgesia provided, but dramatically increases the incidence of pruritus (Figure 2). From 
previous studies it would be expected that at this dose, $43 \%$ of women would experience pruritus, 12\% vomiting and 10\% nausea (Dahl, Jeppesen et al. 1999).

In this study, we had a considerably higher incidence of pruritus of $87 \%$ in the intrathecal morphine group versus $56 \%$ in the oral oxycodone group, which was also more severe in the intrathecal morphine group (Table 7). The incidence in the intrathecal morphine group is higher than that expected from Dahl et al, which was a meta-analysis of trials using intrathecal morphine. The incidence of pruirtus recorded is very dependent on the methods of evaluation and whether direct questioning is used. Our high incidence is consistent with other studies (refs) and may reflect that patients were asked if they had "any" pruritus in the preceding 24 hours, with no differentiation for the time that the pruritus occurred. Given that intrathecal fentanyl, which was administered in combination with intrathecal bupivacaine for the operative procedure, can cause early post-operative pruritus (Gulhas, Erdil et al. 2007), the figure may document the incidence of pruritus resulting from both opioids. This may also explain why there was still a high incidence of pruritus in the oxycodone group, even though it was clinically and statistically significantly lower.

The exact mechanisms of opioid induced pruritus have not been fully elucidated, but pruritus generally results from activation of mu-opioid and 5-hydoxytryptamine ${ }_{3}$ receptors as well as non-nociceptive neurons in the medulla and dorsal horn of the spinal cord, particularly in the trigeminal nerve distribution (Gurkan and Toker 2002). The lower incidence of pruritus in the oral oxycodone group was an expected finding because intrathecal opioids are more commonly associated with pruritus. This may be because of lower opioid concentrations in the central nervous systems structures involved in the generation of this symptom. It is possible that a lower dose of intrathecal morphine might have been associated with a reduced incidence of side effects, although the high rate of supplementation in the intrathecal morphine group would suggest that the benefits of lower pruritus would be mitigated by potentially higher rates of analgesia supplementation.

The incidence of nausea in this study is higher than that predicted by Dahl et al, who, in their systematic review of trials, suggested that the incidence of nausea would be $16 \%$ with a $100 \mathrm{mcg}$ dose of intrathecal morphine and $5 \%$ in control subjects, with the incidence of vomiting being $21 \%$ with $100 \mathrm{mcg}$ of intrathecal 
morphine and 9\% in control subjects (Dahl, Jeppesen et al. 1999). While we did not collect data on the incidence of vomiting in this study, the incidence of nausea was $46 \%$ in the intrathecal morphine group and $34 \%$ in the oral oxycodone group.

It is unclear as to the reasons why the incidence of nausea was higher in our study overall in comparison to the work by Dahl et al., except that the latter is likely to be an underestimate as a result of pooling data from trials, many of which did not investigate either pruritus or nausea symptoms in detail. For antiemetic prophylaxis, we chose to use intraoperative dexamethasone secondary to its proven efficacy and prolonged duration of action (Henzi, Walder et al. 2000). Recent work specific to the obstetric population receiving intrathecal morphine would suggest that it might not be the best choice of agent in this setting. In $2007 \mathrm{Wu}$ et al published the results of a study in which they compared prophylactic dexamethasone and droperidol, or their combination, for PONV prophylaxis in women receiving $200 \mathrm{mcg}$ of intrathecal morphine for post-caesarean analgesia (Wu, Lo et al. 2007). In their study they found that dexamethasone was relatively ineffective for early $(0-6 \mathrm{~h})$ post-operative nausea and vomiting, a finding supported by work from Nortcliffe et al in 2003 (Nortcliffe, Shah et al. 2003). This is in contrast to the results seen for epidural morphine, perhaps because these symptoms occur later with epidural morphine compared with intrathecal morphine (Wu, Lo et al. 2007).

Dexamethasone has a prolonged biological half life of 36-72 h (Tan, Liu et al. 2001) and for chemotherapy induced nausea and vomiting it provides better control of delayed emesis (beyond $24 \mathrm{~h}$ ) (Tavorath and Hesketh 1996), which is another potential explanation as to why it might not have resulted in effective prophylaxis in our study.

Sedation and respiratory depression is a concern when using opioids for postoperative analgesia. Respiratory depression is of particular concern with intrathecal morphine, although the actual incidence in the obstetric population appears to be very low (Carvalho 2008; Kato, Shimamoto et al. 2008). Because of the low lipid solubility of morphine, when administered intrathecally it can spread rostrally to brainstem control centres and contribute to respiratory depression (Bromage, Camporesi et al. 1982; Chaney 1995). This is most likely to occur within the first 618 hours post-operatively (Carvalho 2008). The obstetric population may be protected from opioid-induced respiratory depression by the protective effects of the respiratory stimulation that occurs in pregnancy secondary due to progesterone 
(Chaney 1995), as well as the lower age of obstetric patients when compared to other patient populations who get intrathecal morphine (for example the elderly orthopaedic population who are likely to be exhibit more sensitivity to opiates).

In this study sedation scores were low, with a median score of 0 at all time points in both groups. There were no documented cases of respiratory depression in the initial 24 hour post-operative period. There was a non-significant trend towards more sedation in the oxycodone group at 18 hours $(\mathrm{P}=0.057)$ and both the global scores for sedation and the AUC for sedation were higher (but not statistically significant) in the oral oxycodone group, which is best demonstrated in the box and whisker blocks for the VAS sedation scores (Figure 18). Of note from the graphical representation of the VAS sedation scores, it is apparent that sedation appeared to occur equally in both the oral oxycodone and intrathecal morphine groups at the 24 hour mark, whereas there was minimal sedation over the preceding period in the intrathecal morphine group but relatively consistent levels in the oxycodone group. A possible explanation for this is the accumulating plasma opioid levels as a result of oral oxycodone, given that increased sedation at 24 hours in the intrathecal morphine group would be consistent with the use of oral opioid analgesia as the efficacy of intrathecal morphine diminishes. 


\subsubsection{Limitations}

This study has a number of limitations. Firstly, the study was designed and therefore powered as a superiority trial. For comparative treatment studies there are essentially three major designs; a superiority trial, in which the hypothesis is that one treatment is superior to another; an equivalence trial, in which the hypothesis is that one treatment provides an equivalent effect to another; and a non inferiority trial, in which the hypothesis is that one treatment is no worse than another (Wang D, Nitsch D et al. 2006).

A superiority study design was chosen, with intrathecal morphine as the "superior" comparative agent for a number of reasons. Both equivalence and non inferiority study designs require a much larger sample size, partly because the Type I and Type II errors are normally set at lower levels, which would have made the trial unfeasible within our unit and budget. Non inferiority trials are very sensitive to sample size and trials that have a large number of dropouts can potentially favour a result showing "no difference" (Wang D, Nitsch D et al. 2006). All three study designs require the definition of a clinically relevant difference in treatment, with which to define either superiority, equivalence or non-inferiority (Pater 2004; Wang D, Nitsch D et al. 2006). Most studies of post-operative analgesia use a 30\% difference in analgesia as the definition of superiority, but there is very limited information about what might be considered "equivalent" or "non inferior" pain relief between two regimens. The use of a superiority trial design limits the conclusions that we can draw from the results. We are not able to conclude that oral oxycodone was equivalent in terms of the analgesia provided, rather, we can only conclude that intrathecal morphine was not superior to oral oxycodone.

It is possible that the fixed dose regimen of oral oxycodone that we chose in this study was not optimal. In this study the dose of oxycodone was based on that used by Davis et al. They administered $50 \mathrm{mg}$ in total over the first 12 post-operative hours, followed subsequently by on demand dosing (Davis, Esposito et al. 2006). We administered $60 \mathrm{mg}$ in total over 24 hours, but allowed generous supplementation with on demand doses. It may be that different combinations of slow and immediate release oxycodone will improve early post-operative pain relief, and decreasing the interval between the fixed doses could also prove of benefit. 
For the management of pruritus we elected to use IV ondansetron as the first line therapy and since this study a recent systematic review has confirmed its efficacy in this regard (Bonnet, Marret et al. 2008). Unfortunately, when it came to the data entry process, we were unable to differentiate those patients who had been administered ondansetron for post-operative nausea and vomiting management from those who had received ondansetron to treat pruritus. Whilst we are still able to compare the incidence of pruritus between the two populations, it is not possible to calculate the number of patients in each group that had pruritus severe enough to require treatment (or patients with post-operative nausea and vomiting severe enough to require treatment).

Ideally we would like to report the results in accordance with the CONSORT statement on the reporting of randomised trials (Moher, Schulz et al. 2001). Unfortunately, prior to this study commencing, we did not keep accurate records of the number of patients that were screened for inclusion in the study. Whilst we can fully report the participant flow through the trial once recruited, we are unable to provide details as to the number of subjects that were approached, the number that refused and the number that met an exclusion criteria and could therefore not be recruited. This was a function of the system determining how our pre-admission clinics run. All the elective caesarean patients are seen at one clinic, attended by between 2-3 anaesthetists. Not all anaesthetists were familiar enough with the study to seek recruitment so usually only one anaesthetist at the clinic was able to recruit. This person would often opt to review those women who met the inclusion criteria. The absence of this data on screening and enrolment has the potential to limit the external validity of the study, although it is considered that the study population is likely to represent the patient population undergoing elective caesarean delivery at our institution.

In terms of our handling of patients who withdrew from the study, we were limited by the manner in which they were withdrawn. For this study when a patient withdrew from participation they also withdrew their consent for further participation. This meant that we were unable to perform an intention to treat analysis because data collection stopped at the point of withdrawal, which in some cases was prior to an active therapy. This was unlikely to have influenced the results, as there were identical numbers of withdrawals in each group and their demographic details were similar to the patients that remained in the study. In the 
future we could include a dropout protocol, such that patients who withdrew were placed onto another standardized method of analgesia (such as a patient controlled intravenous or epidural analgesia) and data could then be collected and analysed on an intention to treat basis.

The study is also limited by the lack of precision in some of the instruments that are used for data collection, particularly in regards to post-operative analgesia and satisfaction. Given that pain is a multidimensional experience, as well as having multiple potential sources post-caesarean delivery, it would be beneficial to develop a specific tool to further examine the various sources of post-caesarean pain, and then to validate such a model. This is discussed in the areas for potential future research below. In regards to patient satisfaction, we are limited in that while we found that the maternal satisfaction score was significantly lower in the oxycodone group, we can only generate theories as to why this may be the case. More detailed validated tools to assess satisfaction with post-operative pain relief specifically are not available but it would have been interesting to obtain comment as to the specific reasons for the lower satisfaction in the oxycodone group. 


\subsubsection{Future studies}

There are a number of potential future studies that could be performed in relation to the result of this study. Firstly, in relation to the oral oxycodone regimen, there is much scope to further refine this. There was a high rate of supplementation with additional analgesia in the oxycodone group $(82 \%)$, with a median dose of additional oxycodone being $25 \mathrm{mg}$. The analgesic efficacy of the regimen may be improved by increasing the dose of the regular post-operative oxycodone administered. Other potential refinements could include shortening the dose interval for the fixed dose component, or increasing the dose administered in recovery, given the trend towards higher pain scores with movement at the $6 \mathrm{~h}$ mark and the higher rest pain scores at the $12 \mathrm{~h}$ mark in the oxycodone group. It would also be interesting to perform a study that again compared both intrathecal morphine and oral oxycodone, but also looked at the combination of intrathecal morphine and fixed dose oral oxycodone, to see if there were any additional benefits to adding intrathecal morphine to a fixed dose oral regimen, given the high supplementation rates and variability in analgesia from intrathecal morphine. The addition of a regular dose of oxycodone might improve pain relief but at the possible expense of higher levels of sedation and risk of respiratory depression.

From the results of our study it is not clear why the maternal satisfaction was lower in the oxycodone group, despite an improved side effect profile. Future studies should more closely examine the reasons for maternal satisfaction with their postoperative analgesia. In this regard, women who are not completely satisfied with their analgesia should be asked to further expand on their reasons. This is important, especially in relation to meeting the needs of future patients and having a consumer based focus. On a similar theme, the questionnaires currently used to measure post-operative pain could be described as somewhat imprecise. Given the multifactorial nature of post-caesarean pain, as discussed in the literature review, it might be useful to examine the various components of post-caesarean pain. This could define whether one particular component was being inadequately treated. Thus another future study would involve the development and validation of a specific post-caesarean pain questionnaire.

The economic implications of an oral approach to post-caesarean analgesia should also be a component of future studies. Given the relative constraints on health care budgets in recent years, the generally increasing costs associated with more modern, 
high technology approaches to health care and the large number of caesareans being performed annually, an oral analgesia regimen, if shown to have considerable cost savings, would be an attractive proposition for health care providers.

In this study the effects of the oral regimen on the neonate were not examined. For such an approach to have widespread acceptability the clinical effects on the neonate should also be analysed. In this regard future studies should include neonatal assessments as part of the overall data collected.

Future studies could also attempt to truly create a patient controlled oral analgesia regimen. The regimen used in this study is best described as fixed dose with ondemand supplementation. Correspondence from one UK centre has described their development of locked cupboards in the patients' rooms from which the patient accesses their own opioid medication, as required (Scott, Cameron et al. 2001). This approach would likely decrease staff workload considerably and potentially improve maternal satisfaction because the woman would no longer be reliant on waiting for a staff member to fetch their supplemental analgesia when requested. It would however involve considerable logistical and administrative work, as documented previously, before being able to be introduced more widely.

Finally, the literature review highlighted the lack of data on the risk factors and incidence of chronic post-caesarean pain. Given the relatively high proportion of patients affected by chronic pain after other surgical procedures as well as the high number of caesareans being performed, there is a need for high quality data in this area. 


\subsection{Conclusions}

In conclusion, this study has shown that a multimodal analgesic regimen based on intrathecal morphine was not superior to one based on oral oxycodone for postcaesarean analgesia. The oral oxycodone regimen conferred generally comparable analgesic efficacy to intrathecal morphine with a lower incidence of pruritus. The intrathecal morphine based regimen was associated with reduced pain scores in the early post-operative period as well as a decreased need for rescue analgesia and higher maternal satisfaction scores. It is unclear why maternal satisfaction was higher in the intrathecal morphine group, although we speculate it was related to more consistent levels of analgesia. A multimodal analgesic regimen that includes oxycodone, paracetamol, parecoxib and diclofenac appears to be an acceptable approach to post-caesarean analgesia. Further refinements to this technique may improve efficacy and the maternal satisfaction. Future studies should look at refining the analgesic technique as well as the methods for assessment, particular of post-caesarean pain and satisfaction. An assessment of the potential economic benefits would appear to be warranted. 


\section{Appendix 1: Copy of Ethical Approval}

Dr Nolan McDonnell

Obstetric Anaesthesia Research Fellow

Department of Anaesthesia and Pain Medicine

King Edward Memorial Hospital

374 Bagot Road

SUBIACO WA 6008

Dear Dr McDonnell

\section{REGISTRATION NUMBER: 1297/EW}

TITLE:

\section{REFERENCE NUMBER:}

\section{MEETING DATE:}

Oral analgesia compared with intrathecal morphine for pain after caesarean delivery: A randomised controlled trial

\section{EC06-57}

\section{September 2006}

The Ethics Committee has recommended approval be given for you to undertake the abovenamed research study. This recommendation has been ratified by King Edward Memorial Hospital.

The Ethics Committee does however wish to be informed immediately of:

I. any untoward effects experienced by any participant in the trial where the effects in degree or nature were not anticipated by the reserchers, and steps taken to deal with these,

II.

substantial changes in the research protocol together with an indication of ethical implications, and

III. other unforseen events.

The Ethics Committee has been charged with the responsibility of keeping the progress of all approved research under surveillance. A copy of the final result progress of committee upon completion of the research or if the must be forwarded to the Committee upou are asked to submit a research is not completed within twelve months you are asked to

The status of the project (completed/in progress/abandoned/not commenced). In the event that a project does not commence within 12 months of being approved by the Ethics Committee the study must be resubmitted to the Committee for approval.

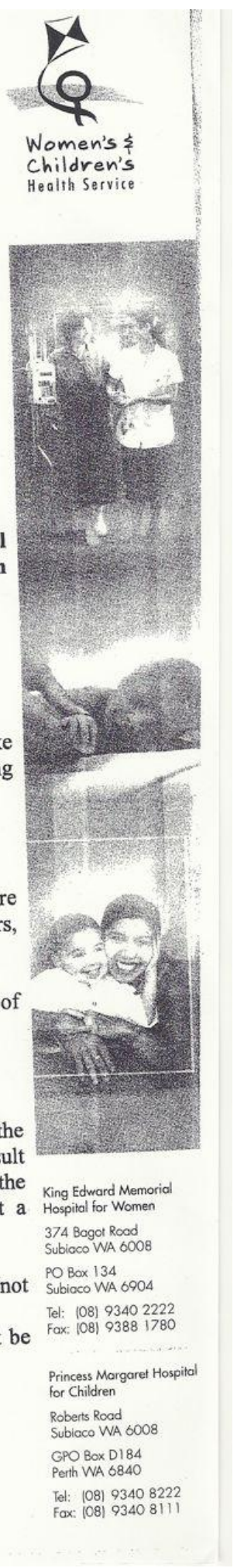


b) Compliance with conditions of ethical approval, including security of records and procedures for consent.

c) Compliance with any special conditions stated by the Ethics Committee as a condition of approval.

d) Results from the study to date, including outcome.

Please note that approval for studies is for three years and the research should be commenced and completed within that period of time. Projects must be resubmitted if an extension of time is required. In the event that a project does not commence within 12 months of being approved by the Ethics Committee the study must be resubmitted to the Committee for approval.

\section{Please quote the above registration number on all correspondence.}

Yours sincerely

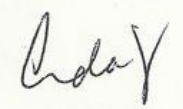

Dr Amanda Frazer

Executive Director

Women's and Newborn Health Service, KEMH

11 September 2006

- The Ethics Committee is constituted, and operates in accordance with the National Health and Medical Research Council's National Statement on Ethical Conduct in Research Involving Humans 


\section{Appendix 2: Patient Information Sheet}

\section{Patient Information Sheet}

\section{Oral opioid analgesia compared with intrathecal morphine for pain relief after caesarean delivery: A randomised controlled trial.}

Principal Investigators:

Dr Nolan McDonnell

Professor Michael Paech

Dr Dorota Doherty

\section{Introduction:}

The Department of Anaesthesia and Pain Medicine at King Edward Memorial Hospital is conducting a study looking at pain relief following caesarean section. Once you have read this information sheet, you should decide whether you wish to take part in the study. Please feel free to discuss this with others, including your partner and family.

You have agreed to have a spinal - epidural anaesthetic for your caesarean section. This is the most commonly used approach for elective caesarean section at King Edward Memorial Hospital.

\section{Study Background:}

There are several methods of pain relief available after caesarean section. All have in common the need to use opioid drugs (like morphine or oxycodone). In addition paracetamol (Panadol) and anti-inflammatory drugs (eg Voltaren) are often used.

The opioid medication can be given to you via a number of different routes. It may be given at the time of your spinal anaesthetic to provide a long-lasting effect, including after the anaesthetic "block' has worn off or via an epidural catheter.

These methods generally provide very effective pain relief but are associated with typical opioid side effects such as nausea, vomiting, itching and drowsiness. Occasionally these side effects interfere with your ability to care for your baby or make your postoperative course somewhat unpleasant.

A new approach to pain relief after caesarean section is to use opioid medications given by mouth more effectively than in the past. A recent study suggested that very good pain relief is possible. The potential advantages are that you do not need to be attached to devices to administer the opioid medication and that the risk of side effects may be less.

In this study we plan to assess the effectiveness of an approach using regular doses of a commonly available oral opioid pain medication called oxycodone, by comparing it with the effects of spinal morphine given at the time of your spinal anaesthetic. 


\section{What does the study involve?}

You will be looked after for your caesarean section in the usual way with a standard spinal - epidural anaesthetic. After your operation pain relief will be provided in one of two different ways and you will be randomly assigned to one of two groups. This means the method of pain relief you have will be determined using a system like the toss of a coin and neither you or your anaesthetist will be able to decide which method of the methods you receive. One group of women will receive morphine administered with their spinal anaesthetic as their primary form of pain relief. The second group will receive regular oral oxycodone capsules as their primary form of pain relief. In both groups this pain relief will be supplemented with regular paracetamol and diclofenac tablets and further oral medications are available if at any time you are not satisfied with the quality of your pain relief.

After the surgery we will measure your pain control, how you are feeling and your recovery from your surgery. The study medications will be stopped 24 hours after your surgery and all patients will then be looked after in the usual way, with oral painrelieving drugs continued for as long as you need.

To ensure the study is valid and not affected by personal bias neither you nor the doctors and nurses looking after you will know what group you are in (this is called blinding). Thus both groups of patients will receive regular oral medications after surgery (every 6 hours), but one group that did not get spinal morphine will also receive regular oral oxycodone, while the other group that did get spinal morphine will receive a placebo tablet that will look identical (the control group). Remember that you are still allowed to ask for other tablet pain-relieving medication at any time.

You will also be visited by the research team at 24 and 48 hours after your surgery. You will be asked about your experience and to again complete brief questionnaires, which you will have completed before the caesarean. These visits should take no longer than five minutes.

\section{Benefits and Risks:}

There is no guarantee that you will benefit from this study. We expect very good pain relief from spinal morphine and hope to find equally good relief (and possibly fewer side effects) from the regular oxycodone method.

The risks associated with participating in this study are very small. Spinal morphine is a commonly used and extremely safe way of getting good pain relief after caesarean section and it will be used at the usual dose and in the usual way. It is possible that you may not experience the same quality of pain relief with oral oxycodone or that side effects are more in one or other group.. The common side effects of opioids are itch (incidence up to 4 in 5 patients), sickness (incidence up to 2 in 5 patients) and drowsiness. Rare effects are unpleasant feelings and hallucinations (incidence up to 1 in 20 patients) and depression of breathing (incidence up to 1 in 250 patients). Everyone in the study will be given a drug to prevent sickness after the operation, will receive treatment for these effects in the usual way and will be monitored in the usual way to detect problems. 


\section{Alternatives:}

There are other alternatives to pain relief after caesarean section. These include epidural opioid medications and intravenous opioids. You may also be given spinal morphine but not be involved in the study if you desire.

\section{Withdrawal from the study:}

Your participation in this study is entirely voluntary. You may withdraw from the study at any time without explanation, and in this case your future treatment will not be affected.

\section{Further information:}

The information collected in this study will be kept confidential, the results of the study may be published but names will not be used.

If you have any questions about the study please contact a Research Nurse (pager 3433 at King Edward Memorial Hospital for Women 9340 2222); or Dr Nolan McDonnell (pager 3226) of Professor Michael Paech (pager 3223).

If you have any complaints about the conduct of this study please contact the Executive Director of Clinical Services, Dr Amanda Frazer on 93408222. 


\section{Appendix 3: Abstract of presentation at the 2008 NZSA/ASA Scientific Congress, Wellington, New Zealand}

Background: Primary post caesarean analgesia based on oral opioid has not been adequately studied. This approach appears to offer benefits in relation to its side effect profile and the avoidance of neuraxial complications.

Methods: In this double-blind, double-dummy, placebo-controlled clinical trial, 120 women were randomised to receive either $20 \mathrm{mg}$ of sustained release oral oxycodone in the recovery room followed by $10 \mathrm{mg}$ of immediate release oxycodone 6 hourly for the first 24 hours (Group O) or $100 \mathrm{mcg}$ of intrathecal morphine at the time of spinal anaesthesia (Group I). Both groups received regular diclofenac and paracetamol post operatively and standardized supplemental analgesia.

Results: 111 women completed the study. Time to first analgesic request was similar between groups $(p=0.597)$. The area under the curve for pain scores to 24 hours did not differ significantly between Group $\mathrm{O}$ and Group I for rest $(\mathrm{p}=0.465)$ or movement pain $(\mathrm{p}=0.533)$.(Table). Additional post operative analgesia was required more often in Group O $(82 \%$ vs. $63 \%, \mathrm{p}=0.034)$, this group also having higher rest pain scores at 12 hours $(\mathrm{p}=0.030)$. Pruritus was more common in Group I $(p=0.001)$. At 24 hours maternal satisfaction with the analgesic regimen was lower in Group $O$ $(\mathrm{p}=0.010)$ but this was not seen at 48 hours $(\mathrm{p}=0.887)$.

Conclusion: Oral oxycodone produced comparable post operative pain relief to intrathecal morphine with a lower incidence of pruritus, but was associated with a lower satisfaction score.

\section{Table}

\begin{tabular}{|c|c|c|c|}
\hline & Group I $(n=55)$ & Group $O(n=56)$ & p-value \\
\hline \multicolumn{4}{|l|}{ VAS pain scores } \\
\hline \multicolumn{4}{|l|}{ At rest } \\
\hline 6 hours & $2(0-3 ; 0-7)$ & $2(1-3 ; 0-10)$ & 0.178 \\
\hline 12 hours & $1(0-2 ; 0-7)$ & $2(1-3 ; 0-7)$ & 0.030 \\
\hline 18 hours & $1(0-2 ; 0-6)$ & $1(0-2 ; 0-5)$ & 0.589 \\
\hline 24 hours & $1(0-2 ; 0-8)$ & $1(0-2 ; 0-8)$ & 0.688 \\
\hline \multicolumn{4}{|l|}{ With movement } \\
\hline 6 hours & $4(3-6 ; 0-10)$ & $5(3-7 ; 1-10)$ & 0.062 \\
\hline 12 hours & $3(2-5 ; 0-8)$ & $4(3-6 ; 0-8)$ & 0.100 \\
\hline 18 hours & $3(2-6 ; 0-8)$ & $3(2-5 ; 0-8)$ & 0.852 \\
\hline 24 hours & $3(2-5 ; 0-9)$ & $3(2-5 ; 0-9)$ & 0.792 \\
\hline \multicolumn{4}{|c|}{ AUC for pain scores to 24 hours } \\
\hline rest & $21(6-35 ; 0-117)$ & $24(12-39 ; 0-84)$ & 0.306 \\
\hline movement & $63(37-96 ; 3-132)$ & $71(42-99 ; 18-135)$ & 0.319 \\
\hline sedation & $6(0-29 ; 0-114)$ & $12(0-42 ; 0-144)$ & 0.237 \\
\hline \multicolumn{4}{|l|}{24 hours post-op } \\
\hline Nausea & $26(46 \%)$ & $19(34 \%)$ & 0.247 \\
\hline Satisfaction & $10(8-10 ; 3-10)$ & $8(7-10 ; 2-10)$ & 0.010 \\
\hline $\begin{array}{l}\text { Additional } \\
\text { analgesia }\end{array}$ & $35(63 \%)$ & $46(82 \%)$ & 0.034 \\
\hline Time to first & 160 (92-230; 23- & 144 (64-218; 20- & 0.597 \\
\hline $\begin{array}{l}\text { analgesia } \\
\text { (mins) }\end{array}$ & 1419) & 1440) & \\
\hline Pruritus & $4(2-5 ; 0-9)$ & $1(0-4 ; 0-9)$ & 0.001 \\
\hline
\end{tabular}




\section{Bibliography}

Abouleish, E., N. Rawal, et al. (1991). "The addition of $0.2 \mathrm{mg}$ subarachnoid morphine to hyperbaric bupivacaine for cesarean delivery: a prospective study of 856 cases." Reg Anesth 16(3): 137-40.

Akhtar-Danesh, N. (2001). "A review of statistical methods for analysing pain measurements." Eur J Pain 5(4): 457-63.

Alhashemi, J. A., Q. A. Alotaibi, et al. (2006). "Intravenous acetaminophen vs oral ibuprofen in combination with morphine PCIA after Cesarean delivery: [L'acetaminophene intraveineux vs l'ibuprofene par voie orale comme adjuvant de la morphine AICP apres une cesarienne]." Can J Anaesth 53(12): 1200-6.

Amabile, C. M. and A. P. Spencer (2004). "Parecoxib for parenteral analgesia in postsurgical patients." Ann Pharmacother 38(5): 882-6.

Anderson, M. (2006). "Diclofenac and post-partum haemorrhage." Int J Obstet Anesth 15(4): 341-2.

Antrobus, H. (2001). "Patient-controlled oramorph--the future?" Anaesthesia 56(2): 195.

Apfelbaum, J. L., T. J. Gan, et al. (2004). "Reliability and validity of the perioperative opioid-related symptom distress scale." Anesth Analg 99(3): 699-709.

Bamigboye, A. and G. Hofmeyr "Local anaesthetic wound infiltration and abdominal nerves block during caesarean section for postoperative pain relief " Cochrane Database of Systematic Reviews 2009, Issue 3. Art. No.: CD006954. DOI: 10.1002/14651858.CD006954.pub2.

Banks, S. and T. Pavy (2001). "A portable, disposable device for patient-controlled epidural analgesia following Caesarean section: evaluation by patients and nurses." Aust N Z J Obstet Gynaecol 41(4): 372-5.

Bay-Nielsen, M., F. M. Perkins, et al. (2001). "Pain and functional impairment 1 year after inguinal herniorrhaphy: a nationwide questionnaire study." Ann Surg 233(1): $1-7$.

Bell, E. A., B. P. Jones, et al. (2002). "Iliohypogastric-ilioinguinal peripheral nerve block for post-Cesarean delivery analgesia decreases morphine use but not opioid-related side effects." Can J Anaesth 49(7): 694-700.

Belzarena, S. D. (1992). "Clinical effects of intrathecally administered fentanyl in patients undergoing cesarean section." Anesth Analg 74(5): 653-7. 
Bisgaard, T., J. Rosenberg, et al. (2005). "From acute to chronic pain after laparoscopic cholecystectomy: a prospective follow-up analysis." Scand I Gastroenterol 40(11): 1358-64.

Bloor, G. K., M. Sinden, et al. (1999). "An audit of single dose epidural diamorphine during elective caesarean section at a district general hospital." Int J Obstet Anesth 8(1): 11-6.

Bloor, G. K., M. Thompson, et al. (2000). "A randomised, double-blind comparison of subarachnoid and epidural diamorphine for elective caesarean section using a combined spinal-epidural technique." Int J Obstet Anesth 9(4): 2337.

Bonnet, F., V. B. Buisson, et al. (1990). "Effects of oral and subarachnoid clonidine on spinal anesthesia with bupivacaine." Reg Anesth 15(4): 211-4.

Bonnet, M. P., E. Marret, et al. (2008). "Effect of prophylactic 5-HT3 receptor antagonists on pruritus induced by neuraxial opioids: a quantitative systematic review." Br J Anaesth 101(3): 311-9.

Bromage, P. R., E. M. Camporesi, et al. (1982). "Rostral spread of epidural morphine." Anesthesiology 56(6): 431-6.

Callesen, T., K. Bech, et al. (1999). "Prospective study of chronic pain after groin hernia repair." Br J Surg 86(12): 1528-31.

Capogna, G., D. Celleno, et al. (1995). "Addition of clonidine to epidural morphine enhances postoperative analgesia after cesarean delivery." Reg Anesth 20(1): 57-61.

Cappelleri, J. C., A. G. Bushmakin, et al. (2009). "Pain Responder Analysis: Use of Area Under the Curve to Enhance Interpretation of Clinical Trial Results." Pain Pract.

Caranza, R., I. Jeyapalan, et al. (1999). "Central neuraxial opioid analgesia after caesarean section: comparison of epidural diamorphine and intrathecal morphine." Int J Obstet Anesth 8(2): 90-3.

Cardoso, M. M., J. C. Carvalho, et al. (1998). "Small doses of intrathecal morphine combined with systemic diclofenac for postoperative pain control after cesarean delivery." Anesth Analg 86(3): 538-41.

Carvalho, B. (2008). "Respiratory depression after neuraxial opioids in the obstetric setting." Anesth Analg 107(3): 956-61.

Carvalho, B., L. Chu, et al. (2006). "Valdecoxib for postoperative pain management after cesarean delivery: a randomized, double-blind, placebo-controlled study." Anesth Analg 103(3): 664-70. 
Carvalho, B., E. Riley, et al. (2005). "Single-dose, sustained-release epidural morphine in the management of postoperative pain after elective cesarean delivery: results of a multicenter randomized controlled study." Anesth Analg 100(4): 1150-8.

Carvalho, B., L. M. Roland, et al. (2007). "Single-dose, extended-release epidural morphine (DepoDur) compared to conventional epidural morphine for post-cesarean pain." Anesth Analg 105(1): 176-83.

Chaney, M. A. (1995). "Side effects of intrathecal and epidural opioids." Can J Anaesth 42(10): 891-903.

Chung, C. J., J. S. Kim, et al. (1998). "The efficacy of intrathecal neostigmine, intrathecal morphine, and their combination for post-cesarean section analgesia." Anesth Analg 87(2): 341-6.

Cohen, S., D. Amar, et al. (1993). "Postcesarean delivery epidural patient-controlled analgesia. Fentanyl or sufentanil?" Anesthesiology 78(3): 486-91.

Cohen, S., I. Lowenwirt, et al. (1998). "Bupivacaine 0.01\% and/or epinephrine 0.5 microg/ml improve epidural fentanyl analgesia after cesarean section." Anesthesiology 89(6): 1354-61.

Cooper, D. W., D. M. Ryall, et al. (1996). "Patient-controlled extradural analgesia with bupivacaine, fentanyl, or a mixture of both, after Caesarean section." Br J Anaesth 76(5): 611-5.

Cooper, D. W., U. Saleh, et al. (1999). "Patient-controlled analgesia: epidural fentanyl and i.v. morphine compared after caesarean section." Br J Anaesth 82(3): 366-70.

Dahl, J. B., I. S. Jeppesen, et al. (1999). "Intraoperative and postoperative analgesic efficacy and adverse effects of intrathecal opioids in patients undergoing cesarean section with spinal anesthesia: a qualitative and quantitative systematic review of randomized controlled trials." Anesthesiology 91(6): 1919-27.

Dahl, V., I. E. Hagen, et al. (2002). "High-dose diclofenac for postoperative analgesia after elective caesarean section in regional anaesthesia." Int J Obstet Anesth 11(2): 91-4.

Dahlgren, G., C. Hultstrand, et al. (1997). "Intrathecal sufentanil, fentanyl, or placebo added to bupivacaine for cesarean section." Anesth Analg 85(6): 1288-93. 
Davies, P. W., M. C. Vallejo, et al. (2005). "Oral herpes simplex reactivation after intrathecal morphine: a prospective randomized trial in an obstetric population." Anesth Analg 100(5): 1472-6.

Davis, K. M., M. A. Esposito, et al. (2006). "Oral analgesia compared with intravenous patient-controlled analgesia for pain after cesarean delivery: a randomized controlled trial." Am J Obstet Gynecol 194(4): 967-71.

Duale, C., C. Frey, et al. (2003). "Epidural versus intrathecal morphine for postoperative analgesia after Caesarean section." Br J Anaesth 91(5): 690-4.

Duthie, D. J. (1998). "Remifentanil and tramadol." Br J Anaesth 81(1): 51-7.

Eisenach, J. C., R. C. Coghill, et al. (2005). "Predictors of pain and analgesic use after cesarean section." Anesthesiology 102, Supp 1: Soap A-10.

Eisenach, J. C., M. De Kock, et al. (1996). "Alpha(2)-adrenergic agonists for regional anesthesia. A clinical review of clonidine (1984-1995)." Anesthesiology 85(3): 655-74.

Eisenach, J. C., S. C. Grice, et al. (1988). "Patient-controlled analgesia following cesarean section: a comparison with epidural and intramuscular narcotics." Anesthesiology 68(3): 444-8.

Fishman, S., J. Condon, et al. (2004). Common opioid-related side effects. Prinicples and practice of pain medicine. C. Warfield and Z. Bajwa, McGraw-Hill.

Fredman, B., A. Shapiro, et al. (2000). "The analgesic efficacy of patient-controlled ropivacaine instillation after Cesarean delivery." Anesth Analg 91(6): 143640.

Gadsden, J., S. Hart, et al. (2005). "Post-cesarean delivery analgesia." Anesth Analg 101(5 Suppl): S62-9.

George, M. J. (2006). "The site of action of epidurally administered opioids and its relevance to postoperative pain management." Anaesthesia 61(7): 659-64.

Ginosar, Y., M. O. Columb, et al. (2003). "The site of action of epidural fentanyl infusions in the presence of local anesthetics: a minimum local analgesic concentration infusion study in nulliparous labor." Anesth Analg 97(5): 1439-45.

Giovannelli, M., N. Bedforth, et al. (2008). "Survey of intrathecal opioid usage in the UK." Eur J Anaesthesiol 25(2): 118-22.

Goh, J. L., S. F. Evans, et al. (1996). "Patient-controlled epidural analgesia following caesarean delivery: a comparison of pethidine and fentanyl." Anaesth Intensive Care 24(1): 45-50. 
Graff, J., M. Arabmotlagh, et al. (2007). "Effects of parecoxib and dipyrone on platelet aggregation in patients undergoing meniscectomy: a double-blind, randomized, parallel-group study." Clin Ther 29(3): 438-47.

Granot, M., L. Lowenstein, et al. (2003). "Postcesarean section pain prediction by preoperative experimental pain assessment." Anesthesiology 98(6): 1422-6.

Grass, J. A., N. T. Sakima, et al. (1997). "A randomized, double-blind, doseresponse comparison of epidural fentanyl versus sufentanil analgesia after cesarean section." Anesth Analg 85(2): 365-71.

Gucev, G., G. M. Yasui, et al. (2008). "Bilateral ultrasound-guided continuous ilioinguinal-iliohypogastric block for pain relief after cesarean delivery." Anesth Analg 106(4): 1220-2.

Gulhas, N., F. A. Erdil, et al. (2007). "Lornoxicam and ondansetron for the prevention of intrathecal fentanyl-induced pruritus." J Anesth 21(2): 159-63.

Gurkan, Y. and K. Toker (2002). "Prophylactic ondansetron reduces the incidence of intrathecal fentanyl-induced pruritus." Anesth Analg 95(6): 1763-6.

Hawkins, J. L., L. M. Koonin, et al. (1997). "Anesthesia-related deaths during obstetric delivery in the United States, 1979-1990." Anesthesiology 86(2): 277-84.

Henzi, I., B. Walder, et al. (2000). "Dexamethasone for the prevention of postoperative nausea and vomiting: a quantitative systematic review." Anesth Analg 90(1): 186-94.

Holt, M. (2000). "Patient-controlled oramorph--the future?" Anaesthesia 55(9): 9334.

Huffnagle, H. J., M. C. Norris, et al. (1996). "Ilioinguinal iliohypogastric nerve blocks--before or after cesarean delivery under spinal anesthesia?" Anesth Analg 82(1): 8-12.

Hyllested, M., S. Jones, et al. (2002). "Comparative effect of paracetamol, NSAIDs or their combination in postoperative pain management: a qualitative review." Br J Anaesth 88(2): 199-214.

Ilett, K. F., M. J. Paech, et al. (2008). "Use of a sparse sampling study design to assess transfer of tramadol and its O-desmethyl metabolite into transitional breast milk." Br J Clin Pharmacol 65(5): 661-6.

Iwasaki, H., J. G. Collins, et al. (1991). "Low-dose clonidine enhances pregnancyinduced analgesia to visceral but not somatic stimuli in rats." Anesth Analg 72(3): 325-9. 
Jakobi, P., I. Solt, et al. (2002). "Over-the-counter oral analgesia for postcesarean pain." Am J Obstet Gynecol 187(4): 1066-9.

Jakobi, P., Z. Weiner, et al. (2000). "Oral analgesia in the treatment of post-cesarean pain." Eur J Obstet Gynecol Reprod Biol 93(1): 61-4.

Kato, R., H. Shimamoto, et al. (2008). "Delayed respiratory depression associated with $0.15 \mathrm{mg}$ intrathecal morphine for cesarean section: a review of 1915 cases." J Anesth 22(2): 112-6.

Kaya, F. N., S. Sahin, et al. (2004). "Epidural neostigmine produces analgesia but also sedation in women after cesarean delivery." Anesthesiology 100(2): 3815.

Kelly, M. C., U. A. Carabine, et al. (1998). "Intrathecal diamorphine for analgesia after caesarean section. A dose finding study and assessment of sideeffects." Anaesthesia 53(3): 231-7.

Lane, S., P. Evans, et al. (2005). "A comparison of intrathecal fentanyl and diamorphine as adjuncts in spinal anaesthesia for Caesarean section." Anaesthesia 60(5): 453-7.

Lavand'homme, P. (2006). "Postcesarean analgesia: effective strategies and association with chronic pain." Curr Opin Anaesthesiol 19(3): 244-8.

Lavand'homme, P. M., F. Roelants, et al. (2007). "Postoperative analgesic effects of continuous wound infiltration with diclofenac after elective cesarean delivery." Anesthesiology 106(6): 1220-5.

Mather, L. E. and M. J. Cousins (2003). "The site of action of epidural fentanyl: what can be learned by studying the difference between infusion and bolus administration? The importance of history, one hopes." Anesth Analg 97(5): 1211-3.

McDonnell, J. G., G. Curley, et al. (2008). "The analgesic efficacy of transversus abdominis plane block after cesarean delivery: a randomized controlled trial." Anesth Analg 106(1): 186-91.

McDonnell, J. G., B. D. O'Donnell, et al. (2007). "Transversus abdominis plane block: a cadaveric and radiological evaluation." Reg Anesth Pain Med 32(5): 399-404.

Mcdonnell, N., P. Kwei, et al. (2007). "A disposable device for patient-controlled intravenous analgesia: Evaluation by patients, nursing and medical staff." Acute Pain 9(2): 71-75. 
Mendoza, T. R., C. Chen, et al. (2004). "The utility and validity of the modified brief pain inventory in a multiple-dose postoperative analgesic trial." Clin J Pain 20(5): 357-62.

Moher, D., K. F. Schulz, et al. (2001). "The CONSORT statement: revised recommendations for improving the quality of reports of parallel-group randomised trials." Lancet 357(9263): 1191-4.

Monagle, J., A. Molnar, et al. (1998). "Oral medication for post-Caesarean analgesia." Aust N Z J Obstet Gynaecol 38(2): 169-71.

Moore, K. (1992). Clinically Orientated Anatomy, Williams and Wilkins.

Munishankar, B., P. Fettes, et al. (2008). "A double-blind randomised controlled trial of paracetamol, diclofenac or the combination for pain relief after caesarean section." Int J Obstet Anesth 17(1): 9-14.

Munsterhjelm, E., T. T. Niemi, et al. (2006). "Influence on platelet aggregation of i.v. parecoxib and acetaminophen in healthy volunteers." $\mathrm{Br}$ J Anaesth 97(2): 226-31.

Myles, P. S., J. O. Hunt, et al. (1999). "Development and psychometric testing of a quality of recovery score after general anesthesia and surgery in adults." Anesth Analg 88(1): 83-90.

National Health and Medical Research Council, Australian Research Council, et al. (2009). National Statement on Ethical Conduct in Human Research, Australian Government.

Ngan Kee, W. D., K. S. Khaw, et al. (1999). "Randomised double-blind comparison of morphine vs. a morphine-alfentanil combination for patient-controlled analgesia." Anaesthesia 54(7): 629-33.

Ngan Kee, W. D., K. K. Lam, et al. (1997). "Comparison of patient-controlled epidural analgesia with patient-controlled intravenous analgesia using pethidine or fentanyl." Anaesth Intensive Care 25(2): 126-32.

Nikolajsen, L., H. C. Sorensen, et al. (2004). "Chronic pain following Caesarean section." Acta Anaesthesiol Scand 48(1): 111-6.

Niruthisard, S., T. Werawataganon, et al. (2007). "Improving the analgesic efficacy of intrathecal morphine with parecoxib after total abdominal hysterectomy." Anesth Analg 105(3): 822-4.

Nortcliffe, S. A., J. Shah, et al. (2003). "Prevention of postoperative nausea and vomiting after spinal morphine for Caesarean section: comparison of cyclizine, dexamethasone and placebo." Br J Anaesth 90(5): 665-70. 
Paech, M. J., J. S. Moore, et al. (1994). "Meperidine for patient-controlled analgesia after cesarean section. Intravenous versus epidural administration." Anesthesiology 80(6): 1268-76.

Paech, M. J., T. J. Pavy, et al. (2000). "Postoperative intraspinal opioid analgesia after caesarean section; a randomised comparison of subarachnoid morphine and epidural pethidine." Int J Obstet Anesth 9(4): 238-45.

Paech, M. J., T. J. Pavy, et al. (2004). "Postcesarean analgesia with spinal morphine, clonidine, or their combination." Anesth Analg 98(5): 1460-6.

Palmer, C. M., S. Emerson, et al. (1999). "Dose-response relationship of intrathecal morphine for postcesarean analgesia." Anesthesiology 90(2): 437-44.

Palmer, C. M., W. M. Nogami, et al. (2000). "Postcesarean epidural morphine: a dose-response study." Anesth Analg 90(4): 887-91.

Pan, P. H. (2006). "Post cesarean delivery pain management: multimodal approach." Int J Obstet Anesth 15(3): 185-8.

Pan, P. H., R. Coghill, et al. (2006). "Multifactorial preoperative predictors for postcesarean section pain and analgesic requirement." Anesthesiology 104(3): 417-25.

Pater, C. (2004). "Equivalence and noninferiority trials - are they viable alternatives for registration of new drugs? (III)." Curr Control Trials Cardiovasc Med 5(1): 8 .

Patolia, D. S., R. L. Hilliard, et al. (2001). "Early feeding after cesarean: randomized trial." Obstet Gynecol 98(1): 113-6.

Pavy, T. J., D. R. Gambling, et al. (1995). "Rectal indomethacin potentiates spinal morphine analgesia after caesarean delivery." Anaesth Intensive Care 23(5): 555-9.

Pavy, T. J., M. J. Paech, et al. (2001). "The effect of intravenous ketorolac on opioid requirement and pain after cesarean delivery." Anesth Analg 92(4): 1010-4.

Perkins, F. M. and H. Kehlet (2000). "Chronic pain as an outcome of surgery. A review of predictive factors." Anesthesiology 93(4): 1123-33.

Ranta, P. O., T. I. Ala-Kokko, et al. (2006). "Incisional and epidural analgesia after caesarean delivery: a prospective, placebo-controlled, randomised clinical study." Int J Obstet Anesth 15(3): 189-94.

Riley, J., E. Eisenberg, et al. (2008). "Oxycodone: a review of its use in the management of pain." Curr Med Res Opin 24(1): 175-92. 
Sabetkasaie, M., S. Vala, et al. (2004). "Clonidine and guanfacine-induced antinociception in visceral pain: possible role of alpha 2/I2 binding sites." Eur J Pharmacol 501(1-3): 95-101.

Sarvela, J., P. Halonen, et al. (2002). "A double-blinded, randomized comparison of intrathecal and epidural morphine for elective cesarean delivery." Anesth Analg 95(2): 436-40.

Scott, S., S. Cameron, et al. (2001). "Maternal self-administration of oral analgesia after Caesarean section." Anaesthesia 56(9): 906-924.

Seaton, S., M. Reeves, et al. (2007). "Oxycodone as a component of multimodal analgesia for lactating mothers after Caesarean section: relationships between maternal plasma, breast milk and neonatal plasma levels." Aust $\mathrm{N}$ Z J Obstet Gynaecol 47(3): 181-5.

Siddik, S. M., M. T. Aouad, et al. (2001). "Diclofenac and/or propacetamol for postoperative pain management after cesarean delivery in patients receiving patient controlled analgesia morphine." Reg Anesth Pain Med 26(4): 310-5.

Swart, M., J. Sewell, et al. (1997). "Intrathecal morphine for caesarean section: an assessment of pain relief, satisfaction and side-effects." Anaesthesia 52(4): $373-7$.

Tan, P. H., K. Liu, et al. (2001). "The effect of dexamethasone on postoperative pain and emesis after intrathecal neostigmine." Anesth Analg 92(1): 228-32.

Tavorath, R. and P. J. Hesketh (1996). "Drug treatment of chemotherapy-induced delayed emesis." Drugs 52(5): 639-48.

Vicente, K. J., K. Kada-Bekhaled, et al. (2003). "Programming errors contribute to death from patient-controlled analgesia: case report and estimate of probability." Can J Anaesth 50(4): 328-32.

Villar, J., E. Valladares, et al. (2006). "Caesarean delivery rates and pregnancy outcomes: the 2005 WHO global survey on maternal and perinatal health in Latin America." Lancet 367(9525): 1819-29.

Wang D, Nitsch D, et al. (2006). Clinical Trials. A practical guide to design, analysis and reporting. London, Remedica.

Wrench, I. J., S. Sanghera, et al. (2007). "Dose response to intrathecal diamorphine for elective caesarean section and compliance with a national audit standard." Int J Obstet Anesth 16(1): 17-21.

Wu, J. I., Y. Lo, et al. (2007). "Prevention of postoperative nausea and vomiting after intrathecal morphine for Cesarean section: a randomized comparison 
of dexamethasone, droperidol, and a combination." Int J Obstet Anesth 16(2): 122-7.

Zohar, E., A. Shapiro, et al. (2006). "Postcesarean analgesia: the efficacy of bupivacaine wound instillation with and without supplemental diclofenac." I Clin Anesth 18(6): 415-21. 